\title{
Oligodendrocyte Progenitor Cells Increase Chi3l1 Secretion in exosome to Activate Myh9 and Promote Proliferation through Connexin47 Connected to Astrocytes
}

\section{Lu Jiang}

Laboratory of Stem Cell and Tissue Engineering, Department of Histology and Embryology, Chongqing Medical University, Chongqing, P.R.China

\section{Xiaoyan Zhang}

Laboratory of Stem Cell and Tissue Engineering, Department of Histology and Embryology,Chongqing Medical University, Chongqing, P.R.China https://orcid.org/0000-0002-2952-978X

\section{Dan Xu}

Department of Neurosurgery, The First Affiliated Hospital of Chongqing Medical University, Chongqing, P.R.China

\section{Wenjin Zhang}

Laboratory of Stem Cell and Engineering, Department of Histology and Embryology, Chongqing Medical University, Chongqing, P.R.China

\section{GuoGuang Peng}

Department of Neurology, The First Affiliated Hospital of Chongqing Medical University, Chongqing,

\section{P.R.China}

\section{Yong Tang}

Laboratory of Stem Cell and Tissue Engineering, Department of Histology and Embryology, Chongqing Medical University, Chongqing, P.R.China

Yan Peng ( $D$ 100433@cqmu.edu.cn)

Laboratory of Stem Cell and Tissue Engineering, Department of Histology and Embryology, Chongqing Medical University, Chongqing, P.R.China

\section{Research}

Keywords: Astrocytes, Oligodendrocyte precursor cells, Cx47, Chi3l1, Myh9

Posted Date: November 12th, 2020

DOl: https://doi.org/10.21203/rs.3.rs-103731/v1 
License: (c) (i) This work is licensed under a Creative Commons Attribution 4.0 International License. Read Full License 


\section{Abstract}

Background: Neurodegenerative diseases, caused by the loss of neurons or myelin sheath, are some of the most important neurological diseases that threaten the health of the elderly. In the CNS, oligodendrocytes (OLs) are the only cells that can form myelin. Astrocytes (ASTs) play a generally beneficial role in remyelination, including the proliferation and differentiation of oligodendrocyte precursor cells (OPCs) to OLs. However, the specific downstream mechanism is unclear.

Methods: This study investigated the proliferation of OPCs in OPCs mono-culture, OPCs culture with ASTs supernatant, and ASTs-OPCs co-culture. Gene Ontology (GO) analysis were used to analyze the differentially expressed genes after transcriptome sequencing of these OPCs. Electron microscope, Nanoparticle Tracking Analysis (NTA), Fluorescence tracing of exosomes and Western blot were used to evaluate the effects of exsomes. Pull-down, co-immunoprecipitation (Co-IP) and mass spectrometry analys were conducted to find the downstream signal proliferation which is transmitted information into OPCs.

Reasults: Direct contact co-culture of ASTs and OPCs promotes the proliferation of OPCs. After Cx47 siRNA interference under ASTs-OPCs co-culture, Chi3l1 secretion in exosome reveals associated decrease, and OPCs proliferation decreased. The cell proliferation induced by Chi3l1 was inhibited after siRNA interfered with Myh9, and the expression of cyclin D1 was also decreased.

Conclusions: These results suggest that ASTs transmit information to OPCs by increasing gap junction channel $\mathrm{Cx} 47$, thereby promoting the secretion of Chi3l1 in exosome of OPCs. The secretory form of Chi3l1 in exosome might be easier to enter the target cell than in extracellular supernatant, which is beneficial to the activation of Myh9 to promote OPCs proliferation. This may be a potential target for drugs rescuing neurodegeneration diseases related to remyelination.

\section{Introduction}

The incidence of neurodegenerative disease is gradually increasing, and neurodegenerative disease has become a major disease type that jeopardizes the physical and mental health of the elderly population.Many studies have confirmed that neurodegenerative diseases, including Alzheimer's disease, Parkinson's disease and multiple sclerosis, are associated with damage and destruction of myelin sheath [1-4]. In the central nervous system (CNS), oligodendrocytes (OLs) are the only cells that can form myelin. The proliferation of OLs depends on the proliferation and differentiation of oligodendrocyte precursor cells (OPCs) [5]. When the body undergoes myelin damage, OPCs that retain proliferative potential proliferate, migrate to the damaged region of the myelin sheath, and differentiate into mature myelin-derived OLs, thereby replacing the myelin sheath that was lost [6, 7]. It is very important to regulate OPCs proliferation for the prevention and rehabilitation of the neurodegenerative disease.

Astrocytes (ASTs) are the glial cells with the largest number and volume in the CNS, supporting, protecting and nourishing other nerve cells. There are extensive gap junctions (GJs) between ASTs and 
OPCs that play a vital role in the process of myelination [8, 9]. Among these $\mathrm{GJ}$ s, the most common is the connexin 47 (Cx47)/Cx43 type [10,11], which, under normal conditions, has an irreplaceable effect on myelin formation [12]. OPCs can connect with ASTs through Cx47 (expressed in OPCs). Loss of Cx47 causes damage to the myelin sheath and severe demyelinating lesions $[13,14]$.

Chitinase 3-like protein 1 (Chi3/1), also known as YKL-40 and gp-39, is a secretory chitinase that lacks chitinase activity [15], and its receptors for exerting biological functions are still unclear. Chi3l1 is expressed in a variety of cells, such as macrophages, fibroblasts, endothelial cells, synovial cells [16-19]. It is highly expressed in a variety of tumor cells, promoting tumor growth, metastasis, invasion [20-22]. For example, Chi3l1 increases the malignancy of tumors in gliomas [23, 24]. Chi3l1 is also expressed in the brain, as an inflammatory marker in Alzheimer's disease [19, 25]. Exosome are small vesicles containing complex protein. Exosome can regulate the biological activity of target cells by carrying proteins, nucleic acids and lipids. There is no report that Chi3l1 exists in exosome.

However, the mechanism that ASTs including the proliferation and differentiation of OPCs is unclear. Studies of GJs Cx47, Chi3/1 in exosome of OPCs are currently lacking, and the relationship between Cx47 and Chi3l1 in exosome has not yet been investigated. Here, transcriptome sequencing, Electron microscope, NTA, pull-down, co-immunoprecipitation (Co-IP) and mass spectrometry results identified expression changes about $\mathrm{C} \times 47$, Chi3/1, exosome, then explored the mechanism between $\mathrm{C} \times 47$ and Chi3l1 in OPCs proliferation.

\section{Materials And Methods}

Experimental animal source. The study has been approved by the Ethics Committee of Chongqing Medical University. Experimental animals were provided by the Experimental Animal Center of Chongqing Medical University.

Collection of B104 supernatant. The frozen B104 cell strain was rapidly dissolved in a $37^{\circ} \mathrm{C}$ water bath, and the dissolved cell suspension was added to a medium containing $12 \%$ fetal bovine serum (FBS, Zhejiang Tianhang Biotechnology, $11011-8511)$. Centrifuged at $1000 \mathrm{rpm}$ for $5 \mathrm{~min}$. The cells were then planted in a petri dish containing $12 \%$ FBS medium, which was changed every other day until the cells were $70 \%-80 \%$ confluent. The medium was replaced with a medium containing $1 \% \mathrm{~N}-2$ supplement (Gibco, A1370701), and cells were incubated for 4 days in the incubator at $37^{\circ} \mathrm{C}$. Then, the supernatant was centrifuged, filtered, collected and stored at $-80^{\circ} \mathrm{C}$.

Primary culture OPCs. The cerebral cells of 1-3-day-old Sprague-Dawley (SD) rats were cultured, and 12\% FBS (Gibco, 10099-141) medium was added. After 4-5 days, the medium was replaced with selective medium which containing $20 \%$ B104 supernatant and $1 \% \mathrm{~N}-2$ supplement. Cells were cultivated for $4-6$ days. OPCs were separated by $0.01 \%$ EDTA digest which cannot digest ASTs because of its weak digestion, and OPCs were seeded in a new medium. 
Primary culture ASTs. The cerebral tissue of newborn SD rats was trypsinized (Gibco, 40127ES60) for $5 \mathrm{~min}$, and the cells were seeded in poly-D-lysine (PDL)-coated dishes and cultured for 5-6 days in a medium containing $12 \%$ FBS.

Experimental groups (Fig. 1).

OPCs mono-culture (group 0). Primary cultured OPCs were digested with 0.01\% EDTA and seeded in petri dishes coated with PDL. A medium containing 1\% N-2 and 20\% B104 supernatant was added, and cells were incubated for 4-6 days.

OPCs cultured with ASTs secretion (group C). ASTs and OPCs were digested with $0.25 \%$ trypsin and $0.01 \%$ EDTA, respectively, and ASTs were seed in the upper chamber of the OPCs. A medium containing $1 \% \mathrm{~N}-2$ and $20 \%$ B104 supernatant was added. Cells were incubated for 4-6 days.

ASTs and OPCs co-culture (group A). After ASTs were seed in PDL-coated dishes for 1 day, OPCs were then seed on the cell surface of ASTs. A medium containing 1\% N-2 and 20\% B104 supernatant was added. Cells were incubated for $4-6$ days. Digest with $0.01 \%$ EDTA when collecting cells.

siRNA interference Cx47 (group Cx47si and group NCsi1). Interfering agents were added to co-cultured ASTs and OPCs according to the instructions of the siRNA Interference Kit (RiboBio). NC siRNA was a disordered RNA used as a control, and the Cx47 interference sequence was CCGAGAAGACTGTCTTCTT. Digest with $0.01 \%$ EDTA when collecting cells.

\section{siRNA interference Cx47 + exosomes ( group Cx47si+)}

Purified OPCs in direct contact with ASTs were cultured in OPC proliferation medium for $24 \mathrm{~h}$. Then, the CX47 siRNA was transfected and about $9 \times 10^{10}$ particles $/ \mathrm{ml}$ of exosomes were added for $24 \mathrm{~h}$ at the same time.

Added exogenous Chi3l1 (group 5Ch and group 10Ch). In these groups, $5 \mathrm{ng} / \mathrm{ml}$ and $10 \mathrm{ng} / \mathrm{ml}$ exogenous Chi3l1 (CLOUD-CLONE CORP, APB463Ra01) was added to the OPCs mono-culture.

siRNA interference Myh9 (groups M1, M2 and M3, group NCsi2). Interfering agents were added to OPCs mono-culture according to the instructions of the siRNA Interference Kit (RiboBio). The interference sequence of Myh9 siRNA1 is: GCCTGTTCTGTGTGGTCAT; siRNA2 is: GCATCGAGTGGAACTTCAT; siRNA3 is: GCGTGACTGGTCTCCTTAA.

Pull-down (group goat and group Ch). Cell lysate was added to OPCs to extract total cellular protein. The proteins were mixed with goat IgG (group goat) (Beyotime, A7007), Chi3l1 antibodies (group Ch) (Santa Cruz, sc-393484) and incubated overnight at $4{ }^{\circ} \mathrm{C}$ with shaking on a shaker. Then, the agarose beads (Santa Cruz, sc-2344) were added to the protein, incubated overnight at $4{ }^{\circ} \mathrm{C}$, and collected. The loading buffer was added to agarose beads and boiled for $10 \mathrm{~min}$, followed by gel electrophoresis. The gel after 
electrophoresis was subjected to Coomassie blue staining, then the protein bands obtained after the staining were cut out and subjected to mass spectrometry to identify the protein species.

Co-immunoprecipitation (Co-IP, group goat and group My). The total cellular protein of OPCs was extracted, mixed with goat IgG and Myh9 antibodies (PRB-440P, Biolegend), incubated overnight at $4{ }^{\circ} \mathrm{C}$ with shaking on a shaker. Then, the agarose beads were added to the protein, incubated overnight at $4{ }^{\circ} \mathrm{C}$, and collected. The loading buffer was then added to the beads and boiled for $10 \mathrm{~min}$. Next, the final obtained protein was analyzed by western blot.

Flow cytometry. OPCs were digested with $0.01 \%$ EDTA and fixed with $75 \%$ alcohol overnight at $4{ }^{\circ} \mathrm{C}$. The cell cycle was detected by flow cytometry.

Immunofluorescence. After the cells were washed with PBS, they were fixed with 4\% paraformaldehyde for 30 min and then incubated with $0.5 \%$ Triton X-100 for 10 min. After a PBS washed, goat serum (Boster, AR0009) was blocked for $1 \mathrm{~h}$. The cells were incubated with the primary antibody (PDGFRa, 1:100, Santa Cruz, sc-338; GFAP, 1:100, Santa Cruz, sc-33673; Cx47, 1:50, Bioworld, BS7447; Chi3l1, 1:50; Myh9, 1:200) overnight at $4{ }^{\circ} \mathrm{C}$, and then with the fluorescent secondary antibody (FITC-labelled goat antirabbit IgG $(\mathrm{H}+\mathrm{L})$, Beyotime, A0562; Cy3-labelled goat anti-mouse IgG $(\mathrm{H}+\mathrm{L})$ Beyotime, A0521) for $2 \mathrm{~h}$ in the dark. The cells were subjected to nuclear staining (DAPI, Beyotime, C1002). Fluorescence was measured by fluorescence microscopy, and the fluorescence intensity of the cells was analyzed by ImageJ.

Transmission electron microscopy (TEM). The exosomes of groups were resuspended in PBS and dropped onto the copper mesh grid of electron microscopy in the form of water droplets. The droplets were retained on the copper grid for $1 \mathrm{~min}$ and fixed in $2 \%$ uranyl acetate for 1-10 min. Then it were dried naturally at room temperature and observed and photographed under $120 \mathrm{kV}$ biotransmission electron microscope.

Nanoparticle tracking analysis (NTA). The exosome samples were appropriately diluted using 1X PBS buffer to measure the particle size and concentration. The exosomes particle size and concentration using NTA at VivaCell Biosceinces with Zeta View PMX 110 (Particle Metrix, Meerbusch, Germany) and corresponding software Zeta View 8.04.02. NTA measurement was recorded and analyzed at 11 positions. The Zeta View system was calibrated using $110 \mathrm{~nm}$ polystyrene particles. Temperature was maintained around $23^{\circ} \mathrm{C}-30^{\circ} \mathrm{C}$.

Western blot. After the total protein of OPCs was extracted, the loading buffer was added and boiled for $10 \mathrm{~min}$. The protein was then subjected to gel electrophoresis, and the strips were cut and electrotransferred. After the strips were blocked with $5 \%$ skim milk powder for $2 \mathrm{~h}$, they were incubated with the primary antibody (Cx47, 1:200; Chi311, 1:200; Myh9, 1:500, Cyclin D1, 1:500, Bioss, bs-20596R; 
GAPDH, Bioss, 1:2000) overnight at $4{ }^{\circ} \mathrm{C}$. After incubation with the secondary antibody for $2 \mathrm{~h}$, the machine captured and developed the strip.

EdU. EdU incubation and fluorescent staining were performed according to the protocol for the EdU kit (RiboBio, C10310-1, C10310-3). Fluorescence microscopy was used to detect cellular fluorescence.

Gene Ontology (GO) analysis. GO analysis of differentially expressed genes was performed using DAVID 6.8 (https://david.ncifcrf.gov), |log2 FC| $\geq 1.5, p<0.05$.

Quantification of exosomes. Exosomes of each group were isolated from $50 \mathrm{ml}$ OPC proliferation medium during a $36 \mathrm{~h}$ culture period and were suspended in $500 \mu \mathrm{l}$ PBS. NTA and western blot analysis Alix of exosomal marker protein for exosomes quantification.

Cellular uptake of exosomes. The exosomes were labeled with PKH26 Red Fluorescent Cell Linker Kit (Sigma-Aldrich, USA). The OPC was coincubated with labeled exosomes at $37^{\circ} \mathrm{C}$ for $24 \mathrm{~h}$. Images were detected via confocal microscopy after mounting using Antifade Mounting Medium with DAPI.

Statistical analysis. One-way analysis of variance was used in this study. All data were processed using SPSS17.

\section{Results}

\section{Direct co-culture of ASTs and OPCs promotes the proliferation of OPCs.}

OPCs mono-cultured were labeled with PDGFRa (Fig. 2A green fluorescence), and ASTs mono-cultured were labeled with GFAP (Fig. 2B red fluorescence). Cell purity reached more than 95\%. OPCs were cultured under three different culture conditions (ASTs-OPCs direct co-culture, OPCs and ASTs secretion co-culture and OPCs mono-culture). EdU staining showed (Fig. 2B and C) that the proportion of neonatal OPCs (red fluorescent-labeled) increased significantly under culture in direct contact with ASTs, and flow cytometry (Fig. 2D and E) revealed that the proportion of OPCs entering the $S$ phase of DNA replication was also significantly increased from $(6.10 \pm 0.27) \%$ in group 0 to $(11.69 \pm 3.27) \%$ in group $C$ and $(21.46 \pm 2.89) \%$ in group A. Therefore, ASTs promote OPCs proliferation through direct contact with OPCs.

A: PDGFRa is specifically expressed in OPCs,labeled with green fluorescence; GFAP is specifically expressed in ASTs $\square$ labeled with red fluorescence. B: Red indicates the nucleus of the neonucleated cells labeled with EdU and blue is the nucleus labeled with DAPI. C: Statistical analysis of the proportion of newborn cells in each group, $\star \star p \rrbracket 0.01 \rrbracket n=9$. D: Statistical analysis of each group cell cycle, ${ }^{\star \star} p \llbracket 0.01 \rrbracket n=$ 9. E: Detection the cell cycle of OPCs under three different culture conditions by flow cytometry.

\section{ASTs promote OPCs proliferation by inducing Cx47 expression in OPCs.}

The OPCs collected under the above three conditions were subjected to transcriptome sequencing, and differentially expressed genes were screened by Gene Ontology (GO) analysis. Genes enriched in channel 
activity (please see Supplementary Material 1) are displayed in a heat map (Fig. 3A). Among these genes, the difference in $\mathrm{Cx} 47$ expression was significant among the three groups. To examine the relationship between Cx47 and OPCs proliferative capacity, the expression level of Cx47 after Cx47 siRNA interference was tested under ASTs-OPCs co-culture conditions. Western blot and immunofluorescence confirmed that Cx47 siRNA successfully reduced the expression of Cx47 (Fig. 3B, C, D and E). Then, we examined the proliferative capacity and cell cycle of OPCs after $\mathrm{Cx} 47$ siRNA interference. The relative number of red fluorescent-labeled neonatal cells was significantly reduced after decreasing Cx47 expression (Fig. 3F and $\mathrm{G}$ ), and flow cytometry revealed that the ability of OPCs to enter the S phase of DNA replication was inhibited with decreased $\mathrm{Cx} 47$ expression (Fig. $3 \mathrm{H}$ and I). Thus, the ability of ASTs to promote the proliferation of OPCs is closely related to $\mathrm{Cx} 47$.

A: Heat map of genes with differential expression on the channel active. B: Western blot detected the expression of $\mathrm{Cx} 47$ in group A, group NCsi1 and group Cx47si. C: Statistical analysis the expression of Cx47 by western blot, $* \star p \otimes 0.01, n=9$. D: Statistical analysis the expression of $\mathrm{Cx} 47$ by immunofluorescence, ${ }^{\star \star} p \otimes 0.01, n=9$. E: Immunofluorescence detection of $\mathrm{Cx} 47$ expression in group $\mathrm{A}$, group NCsi1 and group Cx47si, Cx47 labeled with green fluorescence. F: Red indicates the nucleus of the neonucleated cells labeled with EdU and blue is the nucleus labeled with DAPI. G: Statistical analysis of the proportion of newborn cells in each group, ${ }^{\star \star} p \bigotimes 0.01, n=6$. H: Statistical analysis of each group cell cycle, ${ }^{\star \star} p \llbracket 0.01$, " $n s "$ not statistically significant, $n=9$. I: Detection the cell cycle of OPCs under three different culture conditions by flow cytometry.

Cx47 regulates the expression of chi3l1 in OPCs under ASTs-OPCs co-culture conditions. Cx47 promotes the proliferation of OPCs. GO analysis of the transcriptome sequencing in OPCs under $\mathrm{Cx} 47$ presence (group A) or absence (group Cx47si) conditions revealed that these proliferation-related genes are mainly concentrated in extracellular exosomes (Fig. 4A). A secondary analysis of these genes (please see Supplementary Material 2) by volcano map suggested that Chi3l1 may be a downstream signal for the Cx47-induced proliferation of OPCs (Fig. 4B). Therefore, we examined the expression of Chi3l1 after Cx47 siRNA interference under ASTs-OPCs co-culture conditions. The western blot results showed that the gray value of the Chi3l1 band decreased from $(1.01 \pm 0.05)$ in group $A$ and $(1.04 \pm 0.22)$ in group NCsi1 to $(0.48 \pm 0.15)$ in group Cx47si (Fig. $4 \mathrm{C}$ and D). The immunofluorescence results also confirmed that the expression of Chi3l1 in OPCs decreased after Cx47 siRNA interference (Fig. 4E and F).

A: GO analysis was performed on the differentially expressed genes of group A and group Cx47si, and combined with the bubble plot and barplot results, the differentially expressed genes of the extracellular exosome were selected for further analysis, $|\log 2(F C)| \geq 1.5$. B: The volcano map analysis of the differential genes enriched in extracellular exosomes. The red and green dots denote the upregulated and downregulated differentially expressed genes, respectively. C: Western blot detected the expression of Chi3I1 after Cx47 siRNA interference. D: Statistical analysis of the expression of Chi3l1 by western blot, $\star \star p \otimes 0.01, n=5$. E: Statistical analysis of the expression of Chi3l1 by immunofluorescence, ${ }^{\star \star} p \rrbracket 0.01, n=9$. $\mathrm{F}$ : Detection of the fluorescence intensity of Chi3l1 after $\mathrm{Cx} 47$ siRNA interference. 


\section{Cx47 regulates the expression of Chi3|1 secretion in exosome to promotes OPCs proliferation.}

Cx47 promoting the secretion of exosomes in OPCs. NTA revealed that the exosomal concentration increased from $9 \times 10^{9}$ particles $/ \mathrm{ml}$ in the CO-OPC group to $8 \times 10^{10}$ particles $/ \mathrm{ml}$ in the ASTs-OPCs group. The exosomal concentration was significantly reduced in the CX47 siRNA group $(8.8 \pm 0.4 \times$ $10^{9}$ particles $\left./ \mathrm{ml}\right)$ compared with those in the ASTs-OPCs $\left(1.5 \pm 0.1 \times 10^{10}\right.$ particles $\left./ \mathrm{ml}\right)$ (Fig. $5 \mathrm{~A}$ and B). The $98.6 \%$ particles of exosome were approximately $135 \mathrm{~nm}$ (Fig. $5 \mathrm{~A}$ and $\mathrm{C}$ ). Exosome of the culture were detected by TEM and western blot analyses. TEM demonstrated vesicles of approximately $100 \mathrm{~nm}$ in diameter (Fig. 5C). Western blot results confirmed the special markers of exosome Alix and CD63 proteins and expression of Chi3l1 in OPCs secreted exosome (Fig. 5D).

Cx47 regulates the expression of Chi3l1 secretion in exosome The exosomal secretion was significantly increase in the ASTs-OPCs group compared with that in the CO-OPC group. The expression of Chi3/1 in exosome of ASTs-OPCs group(10.29 \pm 1.71$)$ is significantly higher than the exosome of supernatant of the CO-OPC group(2.87 \pm 0.31 )(Fig. 5C\&D). SiRNA CX47 significantly reduce the expression of Chi3l1 in exosome of the siRNA CX47 group $(2.09 \pm 0.58)$ compared with in the ASTs-OPCs group(Fig. 5E\&G).

SiRNA CX47 significantly decreased the proliferative capacity of OPCs. The results showed a lower percentage of S phase OPCs in group CX47si(17.59 $\pm 0.64 \%)$ than those in groups ASTs-OPCs $(26.78 \pm$ $0.78 \%$ ). The ability of siRNA CX47 to inhibit proliferation OPCs was alleviated by exosomes supplementation. Exosomes labeled with PKH26 were coincubated in the ASTs-OPCs group for $24 \mathrm{~h}$ to evaluate whether they could be internalized by cells. PKH26 was observed to transfer into cells (Fig. $5 \mathrm{I}$ ). The proportion of OPCs in S phase increased from $17.38 \pm 0.32 \%$ in CX47si group to $22.07 \pm 0.15 \%$ in the CX47si + group (Fig. 5J and K). These results indicate that exosome play a vital role in the proliferation of OPCs.

A: Nanoparticle tracking analysis (NTA). The exosome samples were measured the particle size and concentration. B: Statistical analysis of the exosomes, ${ }^{\star \star} p \otimes 0.01, n=9$. C:Transmission electron microscopy detection of exosomes. D: Western blot analysis of exosomes (Exo) and cell lysates (CL). E\&F: Western blot analysis of Chi3l1 in exosome of Co-OPCs and ASTs-OPCs groups, ${ }^{\star \star} p \otimes 0.01, n=6$. G\&H:Western blot analysis of Chi3l1 in exosome of ASTs-OPCs and SiRNA CX47 groups $\llbracket \star \star p \llbracket 0.01, n=6$. I: Exosomes labeled with PKH26 were observed.. J\&K: Flow cytometry detected the proportion of each cell cycle, ${ }^{\star \star} p \otimes 0.01, n=6$.

\section{Exogenous chi3l1 promotes OPC proliferation.}

Many studies have reported that Chi3l1 can promote tumor cell growth during tumor progression [22, 26], but the role of OPCs has not been reported. To test whether Chi3/1 also promotes cell proliferation in OPCs, $5 \mathrm{ng} / \mathrm{ml}$ and $10 \mathrm{ng} / \mathrm{ml}$ exogenous Chi3/1 were added to OPCs under mono-culture conditions. Exogenous Chi3l1 increased the proportion of green fluorescent-labeled neonatal OPCs (Fig. 6A and B) and the proportion of OPCs entering the $S$ phase of the cell cycle (Fig. $6 \mathrm{C}$ and D), confirming that Chi3I1 also promotes cell proliferation in OPCs. 
A: After addition of exogenous Chi3l1 at $5 \mathrm{ng} / \mathrm{ml}$ and $10 \mathrm{ng} / \mathrm{ml}$, respectively, EdU detected the proliferative capacity of OPCs, and the neonatal OPCs were labeled with green fluorescence. B: Statistical analysis of the proportion of new cells detection by EdU, ${ }^{\star \star} p \otimes 0.01, n=9$. C: Statistical analysis of each group cell cycle, ${ }^{\star} p \not \otimes 0.01,{ }^{\star} p \otimes 0.05, n=5$. D: Flow cytometry detected the proportion of each cell cycle after adding exogenous Chi3l1.

\section{Chi3l1 may bind Myh9 to transmit information to OPCs.}

Chi311 is an exocrine glycoprotein with a molecular weight of $40 \mathrm{kDa}$. Its role in promoting cell proliferation must depend on the receptor, but Chi311 has no specific receptor. A pull-down experiment was performed on the protein homogenate of OPCs using agarose beads combined with a Chi3l1 antibody, and the protein bound to the agarose beads was electrophoresed. The gel was then stained with Coomassie blue, and the bands were cut for mass spectrometry for identification of the protein species (Fig. 7A). A total of 111 proteins (please see Supplementary Material 3) were detected by mass spectrometry, of which Myh9 had the highest degree of peptide matching (Fig. 7B). Then, we used the Myh9 antibody for Co-IP experiments and performed western blot on the pulled-down proteins, revealing that the protein pulled down by the Myh9 antibody contained Chi3l1 (Fig. 7C). The experiment proves that Chi3l1 is able to bind Myh9.

A: The electrophoresis gel of the pull-down protein was stained with Coomassie brilliant blue. Myh9 was identified in the strip labeled "a". B: The map of Myh9 analyzed by mass spectrometry with the amino acid sequence shown above the map. C: Western blot of the protein pulled down by the Myh9 antibody with goat IgG used as a control.

\section{Chi3l1 promotes OPC proliferation through Myh9.}

The addition of $10 \mathrm{ng} / \mathrm{ml}$ exogenous Chi3l1 was shown to promote the proliferation of OPCs. On this basis, Myh9 siRNA interference was performed to observe whether OPCs proliferation was inhibited. The three siRNA interference sequences in the siRNA kit were screened and verified to select the siRNA sequence with the best interference effect. The western blot results (Fig. 8A and C) showed that the M1 interference sequence was most effective, which was confirmed by immunofluorescence (Fig. 8D and E).

Here, $10 \mathrm{ng} / \mathrm{ml}$ exogenous Chi3l1 protein was added to mono-culture OPCs, and M1 siRNA was used to knockdown expression of Myh9. EdU staining (Fig. 8F and G) showed that M1 siRNA decreased the number of green fluorescent-labeled neonatal cells, and flow cytometry (Fig. $8 \mathrm{I}$ and $\mathrm{J}$ ) revealed that M1 siRNA suppressed the ability of OPCs to enter the $S$ phase. Western blot (Fig. 8B and H) showed that the expression level of cyclin D1 decreased with the decrease Myh9 expression in OPCs. The above experiments demonstrated that inhibition of Myh9 expression inhibits the OPC proliferation induced by Chi3l1.

A: Western blot detected the expression of Myh9 after siRNA interfered with Myh9. B: Western blot detected the expression of cyclin D1 after siRNA interfered with Myh9. C: Statistical analysis of Myh9 
expression after Myh9 siRNA interference detected by western blot, ${ }^{\star} p \llbracket 0.05,{ }^{\star \star} p \llbracket 0.01, n=5$. D: Statistical analysis of Myh9 expression after Myh9 siRNA interference detected by immunofluorescence, ${ }^{*} p \otimes 0.05$, $\star \star p \llbracket 0.01, n=5$. E: Immunofluorescence detected the fluorescence intensity of Myh9 after Myh9 siRNA interference and Myh9 was labeled with green fluorescently. F: EdU detected the proportion of nascent OPCs labeled with green fluorescently. G: Statistical analysis the proportion of new OPCs after Myh9 siRNA interference in OPCs under the adding exogenous Chi3l1 conditions, ${ }^{\star} p \otimes 0.05,{ }^{\star} * p \otimes 0.01, n=9 . \mathrm{H}$ : Statistical analysis of the expression of cyclin D1 after Myh9 siRNA interference detected by western blot,

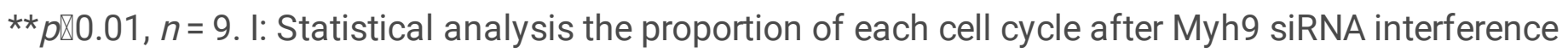
detected by flow cytometry, ${ }^{\star} p \llbracket 0.05,{ }^{\star} p \otimes 0.01, n=9$. J: Flow cytometry detected each cell cycle of OPCs added exogenous Chi3l1 after Myh9 siRNA interference.

\section{Discussion}

Neurodegenerative diseases, caused by the loss myelin sheath, are some of the most important neurological diseases that threaten the health of the elderly. In the CNS, OLs are the only cells that can form myelin [27]. The proliferation of OLs depends on the proliferation and differentiation of OPCs. It is very important to regulate OPCs proliferation for the prevention and rehabilitation of the neurodegenerative disease. The interaction between glial cells exerts a regulatory function on the growth of glial cells themselves. As the most abundant glial cells, ASTs have an important influence on the proliferation of OPCs under both pathological and physiological conditions [28-31]. Under normal conditions, ASTs provide nutrients and necessary cytokines for the growth and survival of OPCs, such as lipids, tumor growth factor $\beta 1$ (TGF $\beta 1$ ), chemokine stromal-derived factor 1 (CXCL12), and fibroblast growth factor receptor 3 (FGFR3) [9, 28, 32-34]. Moreover, the effect of ASTs on OLs may be bidirectional regulation. For example, in MS, ASTs can promote inflammation to strengthen myelin damage on the one hand, and on the other hand protect OLs and axons from inflammatory damage [35]. It can be seen that when the body undergoes demyelination, ASTs can regulate the growth of OLs to compensate for the loss of myelin[31, 36-40].

A similar phenomenon was found in this study, which examined the proliferation ability of OPCs under conditions of OPCs mono-culture, ASTs supernatant culture, and ASTs-OPCs co-culture. The OPCs in direct contact with ASTs were found to proliferate vigorously, indicating that the chemical channels produced by the direct contact between ASTs and OPCs can inevitably transmit information that promotes OPC proliferation and cannot be replaced by AST supernatant.

GJs are information channels formed by hemichannel protein subunits or hexamer linkers between adjacent cells, allowing small molecules and ions to pass through [41, 42]. Their molecular weights range from 25-62 kDa and are used to name different gap junction proteins [43]. There are $21 \mathrm{GJ}$ proteins expressed in the human CNS [44], of which OLs mainly express three types, including Cx47, Cx32 and Cx29, while ASTs mainly express Cx43, Cx30 and Cx26 [45, 46]. In addition to the GJs formed between OLs and their own cells, Cx47/Cx43 and Cx32/Cx30 are mainly formed between OLs and ASTs, whereas Cx29 does not participate in the formation of GJs with ASTs [47]. Cx47 is expressed in the 
oligodendrocyte cell line and is a hemichannel that can be linked to Cx43 expressed by ASTs in the brain [48]. GO analysis of differentially expressed genes in OPCs under three culture conditions, screened 47 differentially expressed genes related to channel activity, which $\mathrm{Cx} 47$ expression was significantly different between the three groups and highly expressed in group A. The stable expression of $\mathrm{Cx} 47$ depends on the presence of ASTs [10,49]. Specifically, Cx47 siRNA interference in ASTs-OPCs co-culture conditions inhibited the proliferative capacity of OPCs, thus confirming that $\mathrm{Cx} 47$ could transmit proliferation information to OPCs.

Biological information analysis of the transcriptome sequencing results revealed that ASTs can increased secretion of Chi3/1 in exosome and exosome of OPCs by Cx47. It suggesting that these increased Chi3l1 in exosome and exosome might be responsible for OPCs proliferation..

Exosome are carriers of information exchange and material transfer between cells. Exosome can participate in cell immune response, migration, differentiation, proliferation and other functions [50]. The results of electron microscopy, immunoblotting and nanoparticle tracking analysis showed that astrocytes adhesion increased the secretion of almost 10 times exosome of OPCs. At the same time, The concentration of Chi3/1 in exosome was also much higher than that Chi3/1 in OPCs. It is suggested that Chi3l1 of exosome is a high concentration transport form. In other words, ASTs can increased autocrine and paracrine of Chi3l1 in exosomes and exosomes of OPCs by $\mathrm{Cx} 47$.

Chi3l1 is a member of the glycoprotein hydrolase 18 family and has the ability to bind chitin but has no enzymatic activity [51-52]. Chi3l1 is a tumor-associated factor that is highly expressed in various tumor cells by activating the phosphatidylinositol-4,5-bisphosphate 3-kinase (PI3K)/AKT and nuclear factor (NF)-KB pathways to promote tumor growth, invasion and metastasis [26, 53-55]. It is also an inflammatory factor that activates the inflammatory response $[19,56-58]$. This study found that Chi3l1 also promotes cell proliferation in OPCs.

Chi3l1 is a secreted protein that inevitably plays a biological role by binding to the corresponding receptor. Because Chi3l1 has no specific receptors in reports, many studies have focused on finding receptors for Chi3/1. Interleukin-13 receptor (IL-13R), CRTH2 and CD44 can interact with Chi3l1 to act as a receptor $[20,59-61]$.

In this study, we used pull-down,mass spectrometry and Co-IP to find the specific combination of Myh9 and Chi3l1. These experiments showed that Myh9 is the downstream signal that Chi3l1 transmits proliferation information into OPCs.

Myh9, also known as nonmuscle myosin heavy chain Ila (NMMHC-IIA), is located on chromosome 22q12.3 [62] and is expressed in many cell types. Myh9 is a cytoskeletal component composed of complex multimeric protein [63]. It is essential for biological processes such as cell migration, division and signal transduction $[62,64-66]$. It also has angiogenesis, vascular remodeling abilities in vascular endothelial cells [66] and promotes tumor invasion, metastasis in some tumors $[64,67]$. Furthermore, Myh9 promotes tumor invasion and metastasis in some tumors [65, 68-70]. In addition, some studies 
have shown that Myh9 also contributes to cell proliferation, cell contraction, adhesion and cytokinesis. In this study, Myh9 siRNA interference on OPCs under the added exogenous Chi3l1 condition revealed that Myh9 knockdown blocked the proliferation of OPCs induced by Chi3l1. The expression of cyclin D1 also decreased with Myh9 siRNA interference.

Our research and other studies have shown that exogenous Chi3l1 can promote the proliferation of OPCs. However, the study also showed that Chi3l1 was secreted in the form of exosome. We speculated that the secretory form of Chi3/1 in exosome might be easier to enter the target cell than in extracellular supernatant, which is beneficial to the activation of Myh9.

In summary, ASTs activate the expression of Chi3l1 in OPCs via gap junction Cx47. Subsequently, the expression and secretion of Chi3l1 in exosome of OPCs is increased. Then, Chi3l1 binds Myh9 of OPCs and transmits proliferation information into the cells to stimulate expression of cyclin D1, thereby promoting OPCs proliferation (Fig. 9). It provides a new understanding for the treatment of neurodegenerative diseases by promoting myelin sheath regeneration.

\section{Conclusions}

ASTs play a generally beneficial role in the proliferation and differentiation of OPCs. However, the specific mechanism is unclear. This study shown that direct contact and secretory exosomes between ASTs and OPCs plays an important role in the proliferation of OPCs. These results suggest that ASTs transmit information to OPCs by increasing gap junction Cx47. More interestingly, ASTs also regulates the expression of Chi3l1 in exosomes of OPCs via gap junction. The exosomes may be the special transport form of Chi3/1 in the cells. It is beneficial to the transmembrane transport of Chi3l1 and the proliferation of OPCs. The Chi3l1 binds Myh9 of OPCs and stimulate expression of cyclin D1. It provides a new understanding for proliferation of OPCs. This may be a potential help for drugs rescuing neurodegeneration diseases related to remyelination.

\section{Abbreviations}

OLs oligodendrocytes; ASTs:Astrocytes; OPCs:oligodendrocyte precursor cells; NTA:Nanoparticle Tracking Analysis; Co-IP:co-immunoprecipitation; CNS:central nervous system; GJs:gap junctions; Chi3l1:Chitinase 3-like protein 1; PDL:poly-D-lysine; GO:Gene Ontology; TGF $\beta 1$ :tumor growth factor $\beta 1$; $C X C L 12$ :chemokine stromal-derived factor 1 ; FGFR3:fibroblast growth factor receptor 3 ; IL-13R:Interleukin-13 receptor; $\mathrm{NF}$ :nuclear factor; NMMHC-IIA:Ilanonmuscle myosin heavy chain Ila.

\section{Declarations}

\section{Acknowledgements}

We thank the Laboratory of Stem Cell and Tissue Engineering for technical support. 


\section{Author's contributions}

Lu Jiang and Xiaoyan Zhang contributed equally to the work as coauthors. Lu Jiang and Xiaoyan Zhang had the key role in experimental design, and collected, organized, and analyzed experimental data. Wenjin Zhang provided intellectual inputs and to analyze the data. All the authors have read and approved to the final text.

\section{Funding}

This study was supported by the Natural Science Foundation of China (NSFC 81671259),the Chongqing Science and Technology Bureau (No.cstc2019jcyj-msxmX0193).

\section{Availability of data and materials}

The data sets used and/or analysed during the current study are available from the corresponding author on reasonable request.

\section{Ethics approval}

The study has been approved by the Ethics Committee of Chongqing Medical University. Experimental animals were provided by the Experimental Animal Center of Chongqing Medical University. All applicable international, national, and institutional guidelines for the care and use of animals were followed.

\section{Consent for publication}

All contributing authors have given their consent for the publication of this study.

\section{Competing interests}

The authors declare that they have no competing interests.

\section{Author details}

${ }^{1}$ Laboratory of Stem Cell and Tissue Engineering, Department of Histology and Embryology, Chongqing Medical University, Chongqing, P.R. China. ${ }^{2}$ Department of Neurosurgery, The First Affiliated Hospital of Chongqing Medical University, Chongqing, P.R. China. ${ }^{3}$ Department of Neurology, The First Affiliated Hospital of Chongqing Medical University, Chongqing, P.R. China

\section{References}

1. Roberts RF, Wade-Martins R, Alegre-Abarrategui J. Direct visualization of alpha-synuclein oligomers reveals previously undetected pathology in Parkinson's disease brain. BRAIN. 2015;138:1642-57.

2. McKenzie AT, Moyon S, Wang M, et al. Multiscale network modeling of oligodendrocytes reveals molecular components of myelin dysregulation in Alzheimer's disease. MOL NEURODEGENER. 
2017;12:82-102.

3. Chang A, Nishiyama A, Peterson J, Prineas J, Trapp BD. NG2-positive oligodendrocyte progenitor cells in adult human brain and multiple sclerosis lesions. J NEUROSCI. 2000;20:6404-12.

4. Nasrabady SE, Rizvi B, Goldman JE, Brickman AM. White matter changes in Alzheimer's disease: a focus on myelin and oligodendrocytes. ACTA NEUROPATHOL COM. 2018;6:22-32.

5. Emery B. Regulation of Oligodendrocyte Differentiation and Myelination. SCIENCE. 2010;330:77982.

6. Gould EA, Busquet N, Shepherd D, et al. Mild myelin disruption elicits early alteration in behavior and proliferation in the subventricular zone. ELIFE. 2018;7:e34783.

7. Ong W, Lin J, Bechler ME, et al. Microfiber drug/gene delivery platform for study of myelination. ACTA BIOMATER. 2018;75:152-60.

8. Stoffels JM, Hoekstra D, Franklin RJ, Baron W, Zhao C. The EllIA domain from astrocyte-derived fibronectin mediates proliferation of oligodendrocyte progenitor cells following CNS demyelination. GLIA. 2015;63:242-56.

9. Zhang Y, Zhang J, Navrazhina K, et al. TGF $\beta 1$ induces Jagged1 expression in astrocytes via ALK5 and Smad3 and regulates the balance between oligodendrocyte progenitor proliferation and differentiation. GLIA. 2010;58:964-74.

10. Orthmann-Murphy JL, Freidin M, Fischer E, Scherer SS, Abrams CK. Two Distinct Heterotypic Channels Mediate Gap Junction Coupling between Astrocyte and Oligodendrocyte Connexins. J NEUROSCI. 2007;27:13949-57.

11. Xiao-Guang Luo \& Sheng-Di Chen. The changing phenotype of microglia from homeostasis to disease. Transl Neurodegener. 2012;1:9.

12. Teubner $B$, Odermatt $B$, Guldenagel $M$, et al. Functional expression of the new gap junction gene connexin47 transcribed in mouse brain and spinal cord neurons. J NEUROSCI. 2001;21:1117-26.

13. Markoullis K, Sargiannidou I, Schiza N, et al. Oligodendrocyte gap junction loss and disconnection from reactive astrocytes in multiple sclerosis gray matter. J Neuropathol Exp Neurol. 2014;73:86579.

14. Masaki K, Suzuki SO, Matsushita T, et al. Extensive loss of connexins in Balós disease: evidence for an auto-antibody-independent astrocytopathy via impaired astrocyte-oligodendrocyte/myelin interaction. ACTA NEUROPATHOL. 2012;123:887-900.

15. Bussink AP, Speijer D, Aerts JMFG, Boot RG. Evolution of Mammalian Chitinase(-Like) Members of Family 18 Glycosyl Hydrolases. GENETICS. 2007;177:959-70.

16. Baldacci F, Lista S, Cavedo E, Bonuccelli U, Hampel H. Diagnostic function of the neuroinflammatory biomarker YKL-40 in Alzheimer's disease and other neurodegenerative diseases. Expert Rev Proteomics. 2017;14:285-99.

17. Kim D, Choi J. Chitinase 3-like-1, a novel regulator of Th1/CTL responses, as a therapeutic target for increasing anti-tumor immunity. 2018;51:207-208. 
18. He CH, Lee CG, Dela Cruz CS, et al. Chitinase 3-like 1 Regulates Cellular and Tissue Responses via IL13 Receptor a2. CELL REP. 2013;4:830-41.

19. Jung YY, Kim KC, Park MH, et al. Atherosclerosis is exacerbated by chitinase-3-like-1 in amyloid precursor protein transgenic mice. THERANOSTICS. 2018;8:749-66.

20. Chen $Y$, Zhang S, Wang Q, Zhang X. Tumor-recruited M2 macrophages promote gastric and breast cancer metastasis via M2 macrophage-secreted CHI3L1 protein. J HEMATOL ONCOL. 2017;10:3649.

21. Kim D, Park H, Lim S, et al. Regulation of chitinase-3-like-1 in T cell elicits Th1 and cytotoxic responses to inhibit lung metastasis. NAT COMMUN. 2018;9:503-17.

22. Zhang W, Kawanishi M, Miyake K, et al. Association between YKL-40 and adult primary astrocytoma. CANCER-AM CANCER SOC. 2010;116:2688-97.

23. Wood MD, Mukherjee J, Pieper RO. Neurofibromin knockdown in glioma cell lines is associated with changes in cytokine and chemokine secretion in vitro. SCI REP-UK. 2018;8:5085-94.

24. Antonelli M, Massimino M, Morra I, et al. Expression of pERK and pAKT in pediatric high grade astrocytomas: Correlation with YKL40 and prognostic significance. NEUROPATHOLOGY. 2012;32:133-8.

25. Querol-Vilaseca M, Colom-Cadena M, Pegueroles J, et al. YKL-40 (Chitinase 3-like I) is expressed in a subset of astrocytes in Alzheimer's disease and other tauopathies. J NEUROINFLAMM. 2017;14:118-28.

26. Libreros S, Iragavarapu-Charyulu V. YKL-40/CHI3L1 drives inflammation on the road of tumor progression. J Leukoc Biol. 2015;98:931-6.

27. Ozgen $\mathrm{H}$, Baron $\mathrm{W}$, Hoekstra D, Kahya N. Oligodendroglial membrane dynamics in relation to myelin biogenesis. CELL MOL LIFE SCI. 2016;73:3291-310.

28. Silver J, Schwab ME, Popovich PG. Central Nervous System Regenerative Failure: Role of Oligodendrocytes, Astrocytes, and Microglia. CSH PERSPECT BIOL. 2015;7:a20602.

29. Xu D, Liu Z, Wang S, Peng Y, Sun X. Astrocytes regulate the expression of Sp1R3 on oligodendrocyte progenitor cells through $\mathrm{Cx} 47$ and promote their proliferation. BIOCHEM BIOPH RES CO. 2017;490:670-5.

30. Arai K, Lo EH. Astrocytes protect oligodendrocyte precursor cells via MEK/ERK and PI3K/Akt signaling. J NEUROSCI RES. 2010;88:758-63.

31. Miyamoto N, Maki T, Shindo A, et al. Astrocytes Promote Oligodendrogenesis after White Matter Damage via Brain-Derived Neurotrophic Factor. J NEUROSCI. 2015;35:14002-8.

32. Camargo N, Goudriaan A, van Deijk AF, et al. Oligodendroglial myelination requires astrocyte-derived lipids. PLOS BIOL. 2017;15:e1002605.

33. Pringle NP. Fgfr3 expression by astrocytes and their precursors: evidence that astrocytes and oligodendrocytes originate in distinct neuroepithelial domains. DEVELOPMENT. 2003;130:93-102. 
34. Patel JR, Williams JL, Muccigrosso MM, et al. Astrocyte TNFR2 is required for CXCL12-mediated regulation of oligodendrocyte progenitor proliferation and differentiation within the adult CNS. ACTA NEUROPATHOL. 2012;124:847-60.

35. Nair A, Frederick TJ, Miller SD. Astrocytes in multiple sclerosis: A product of their environment. CELL MOL LIFE SCI. 2008;65:2702-20.

36. Kıray H, Lindsay SL, Hosseinzadeh S, Barnett SC. The multifaceted role of astrocytes in regulating myelination. EXP NEUROL. 2016;283:541-9.

37. Nobuta H, Ghiani CA, Paez PM, et al. STAT3-Mediated astrogliosis protects myelin development in neonatal brain injury. ANN NEUROL. 2012;72:750-65.

38. Schulz K, Kroner A, David S. Iron Efflux from Astrocytes Plays a Role in Remyelination. J NEUROSCl. 2012;32:4841-7.

39. Annunziato L, Boscia F, Pignataro G. Ionic transporter activity in astrocytes, microglia, and oligodendrocytes during brain ischemia. J Cereb Blood Flow Metab. 2013;33:969-82.

40. Fulmer CG, VonDran MW, Stillman AA, et al. Astrocyte-Derived BDNF Supports Myelin Protein Synthesis after Cuprizone-Induced Demyelination. J NEUROSCI. 2014;34:8186-96.

41. Imbeault S, Gauvin LG, Toeg HD, et al. The extracellular matrix controls gap junction protein expression and function in postnatal hippocampal neural progenitor cells. BMC NEUROSCI. 2009;10:13-30.

42. Belousov AB, Fontes JD, Freitas-Andrade M, Naus CC. Gap junctions and hemichannels: communicating cell death in neurodevelopment and disease. BMC CELL BIOL. 2017;18:4-15.

43. Evans WH, De Vuyst E, Leybaert L. The gap junction cellular internet: connexin hemichannels enter the signalling limelight. BIOCHEM J. 2006;397:1-14.

44. Kleopas A, Kleopa. Jennifer Orthmann-Murphy, Irene Sargiannidou. Gap junction disorders of myelinating cells. Rev Neurosci. 2010;21(5):397-419.

45. Kim MS, Gloor GB, Bai D. The distribution and functional properties of Pelizaeus-Merzbacher-like disease-linked $\mathrm{C} \times 47$ mutations on $\mathrm{C} \times 47 / \mathrm{C} \times 47$ homotypic and $\mathrm{C} \times 47 / \mathrm{C} \times 43$ heterotypic gap junctions. BIOCHEM J. 2013;452:249-58.

46. Li X, lonescu AV, Lynn BD, et al. Connexin47, connexin29 and connexin32 co-expression in oligodendrocytes and $\mathrm{Cx} 47$ association with zonula occludens-1 (ZO-1) in mouse brain. NEUROSCIENCE. 2004;126:611-30.

47. Altevogt BM. Four Classes of Intercellular Channels between Glial Cells in the CNS. J NEUROSCl. 2004;24:4313-23.

48. Kleopa KA, Sargiannidou I. Connexins, gap junctions and peripheral neuropathy. NEUROSCI LETT. 2015;596:27-32.

49. Wasseff SK, Scherer SS. Cx32 and Cx47 mediate oligodendrocyte:astrocyte and oligodendrocyte:oligodendrocyte gap junction coupling. NEUROBIOL DIS. 2011;42:506-13. 
50. Suresh M, Hong J, Richard JS. Exosomes: Extracellular Organelles Important in Intercellular Communication. J Proteomics. 2010;73(10):1907-20.

51. Tingting Xiao W, Zhang BJ, et al. The role of exosomes in the pathogenesis of Alzheimer'disease. Transl Neurodegener. 2017;6:3.

52. Zaheer-ul-Haq, Dalal P, Aronson NJ, Madura JD. Family 18 chitolectins: comparison of MGP40 and HUMGP39. Biochem Biophys Res Commun. 2007;359:221-6.

53. Thorn AP, Daugaard S, Christensen LH, Christensen IJ, Petersen MM. YKL-40 protein in osteosarcoma tumor tissue. APMIS. 2016;124:453-61.

54. Horbinski C, Wang G, Wiley CA. YKL-40 is directly produced by tumor cells and is inversely linked to EGFR in glioblastomas. Int J Clin Exp Pathol. 2010;3:226-37.

55. SALOMON J, PIOTROWSKA A, MATUSIAK A, DZIĘGIEL P, SZEPIETOWSKI JC. Chitinase-3-like Protein 1 (YKL-40) Expression in Squamous Cell Skin Cancer. ANTICANCER RES. 2018;38:4753-8.

56. Llorens $F$, Thüne K, Tahir W, et al. YKL-40 in the brain and cerebrospinal fluid of neurodegenerative dementias. MOL NEURODEGENER. 2017;12:83-104.

57. Recklies AD, Ling H, White C, Bernier SM. Inflammatory Cytokines Induce Production of CHI3L1 by Articular Chondrocytes. J BIOL CHEM. 2005;280:41213-21.

58. Bonneh-Barkay D, Bissel SJ, Kofler J, et al. Astrocyte and Macrophage Regulation of YKL-40 Expression and Cellular Response in Neuroinflammation. BRAIN PATHOL. 2012;22:530-46.

59. Geng B, Pan J, Zhao T, et al. Chitinase 3-like 1-CD44 interaction promotes metastasis and epithelialto-mesenchymal transition through $\beta$-catenin/Erk/Akt signaling in gastric cancer. J EXP CLIN CANC RES. 2018;37:208-28.

60. He CH, Lee CG, Dela Cruz CS, et al. Chitinase 3-like 1 Regulates Cellular and Tissue Responses via IL13 Receptor a2. CELL REP. 2013;4:830-41.

61. Zhou Y, He CH, Herzog EL, et al. Chitinase 3-like-1 and its receptors in Hermansky-Pudlak syndrome-associated lung disease. J CLIN INVEST. 2015;125:3178-92.

62. Pecci A, Ma X, Savoia A, Adelstein RS. MYH9: Structure, functions and role of non-muscle myosin IIA in human disease. GENE. 2018;664:152-67.

63. Heissler SM, Manstein DJ. Nonmuscle myosin-2: mix and match. CELL MOL LIFE SCI. 2013;70:1-21.

64. Liang $\mathrm{S}, \mathrm{He} L$, Zhao X, et al. MicroRNA let-7f inhibits tumor invasion and metastasis by targeting MYH9 in human gastric cancer. PLOS ONE. 2011;6:e18409.

65. Arora PD, Wang Y, Bresnick A, Janmey PA, McCulloch CA. Flightless I interacts with NMMIIA to promote cell extension formation, which enables collagen remodeling. MOL BIOL CELL. 2015;26:2279-97.

66. Morrison AR, Yarovinsky TO, Young BD, et al. Chemokine-coupled $\beta 2$ integrin-induced macrophage Rac2-Myosin IIA interaction regulates VEGF-A mRNA stability and arteriogenesis. J Exp Med. 2014;211:1957-68. 
67. Chiu H, Chang T, Huang C, Chao Y, Hsu JTA. EGFR and myosin II inhibitors cooperate to suppress EGFR-T790M-mutant NSCLC cells. MOL ONCOL. 2012;6:299-310.

68. Nguyen-Ngoc K, Silvestri VL, Georgess D, Fairchild AN, Ewald AJ. Mosaic loss of non-muscle myosin IIA and IIB is sufficient to induce mammary epithelial proliferation. J CELL SCI. 2017;130:3213-21.

69. Sundvold H, Sundvold-Gjerstad V, Malerød-Fjeld H, et al. Arv1 promotes cell division by recruiting IQGAP1 and myosin to the cleavage furrow. CELL CYCLE. 2016;15:628-43.

70. Casalou C, Seixas C, Portelinha A, et al. Arl13b and the non-muscle myosin heavy chain IIA are required for circular dorsal ruffle formation and cell migration. J CELL SCI. 2014;127:2709-22.

\section{Figures}

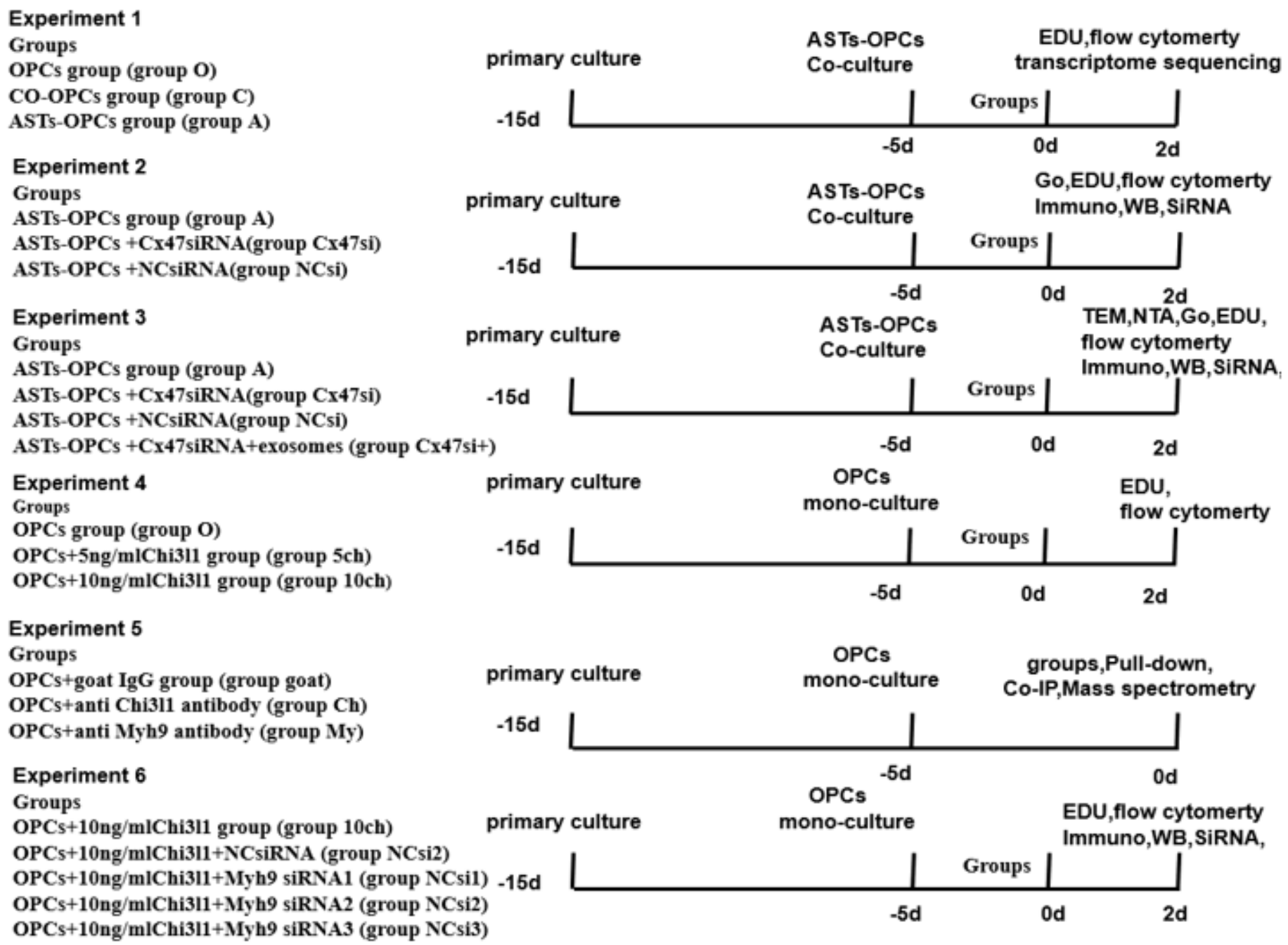

Figure 1

Cell group classification and experimental design. 


\section{Experiment 1}

Groups

OPCs group (group $\mathbf{O}$ )

CO-OPCs group (group C)

ASTs-OPCs group (group A)

Experiment 2

Groups

ASTs-OPCs group (group A)

ASTs-OPCs + Cx47siRNA(group Cx47si)

ASTs-OPCs +NCsiRNA(group NCsi)

\section{Experiment 3}

Groups

ASTs-OPCs group (group A)

ASTs-OPCs + Cx47siRNA(group Cx47si)

ASTs-OPCs + NCsiRNA(group NCsi)

ASTs-OPCs +Cx47siRNA+exosomes (group Cx47si+)

\section{Experiment 4}

Groups

OPCs group (group $\mathbf{O}$ )

OPCs $+5 \mathrm{ng} / \mathrm{mlChi3l1}$ group (group $5 \mathrm{ch}$ )

OPCs+10ng/mlChi3l1 group (group 10ch)

\section{Experiment 5}

\section{Groups}

OPCs+goat IgG group (group goat)

OPCs+anti Chi3l1 antibody (group Ch)

OPCs+anti Myh9 antibody (group My)

\section{Experiment 6}

Groups

OPCs+10ng/mlChi3l1 group (group 10ch)

OPCs+10ng/mlChi3!1+NCsiRNA (group NCsi2)

OPCs+10ng/mlChi3l1+Myh9 siRNA1 (group NCsil) $-15 \mathrm{c}$

OPCs+10ng/mlChi3l1+Myh9 siRNA2 (group NCsi2)

OPCs+10ng/mlChi3l1+Myh9 siRNA3 (group NCsi3)

$-15 d$ $-15 d$
ASTs-OPCs EDU,flow cytomerty

Co-culture transcriptome sequencing

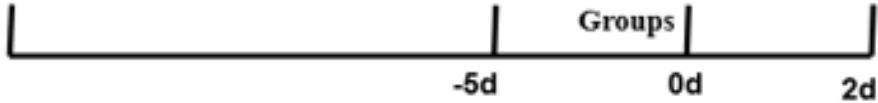

primary culture

ASTs-OPCs

Co-culture

Go,EDU,flow cytomerty Immuno,WB,SIRNA

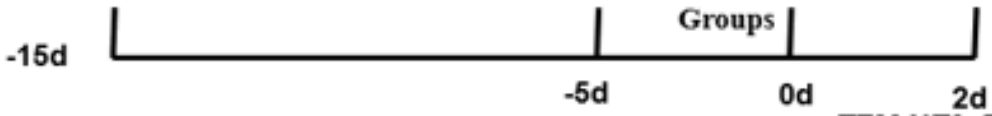

ASTs-OPCs

$\begin{array}{ll}\text { primary culture } & \text { ASTs-OPCs } \\ \text { Co-culture }\end{array}$

TEM,NTA, Go,EDU, flow cytomerty

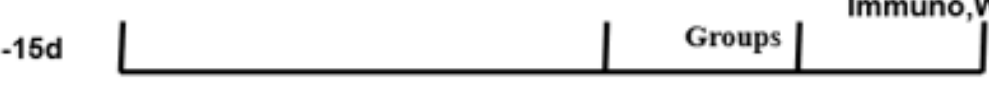

primary culture

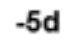

od

2d

OPCs

mono-culture

$-15 d$

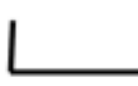

primary culture

OPCs mono-culture groups,Pull-down,

$-15 d$

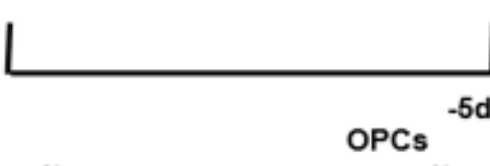

primary culture

mono-culture
EDU,

Groups

Od

2d

Co-IP,Mass spectrometry

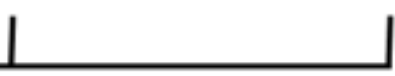

Od

EDU,flow cytomerty Immuno,WB, SiRNA, flow cytomerty

L

\section{Figure 1}

Cell group classification and experimental design. 


\section{Experiment 1}

Groups

OPCs group (group $\mathbf{O}$ )

CO-OPCs group (group C)

ASTs-OPCs group (group A)

Experiment 2

Groups

ASTs-OPCs group (group A)

ASTs-OPCs + Cx47siRNA(group Cx47si)

ASTs-OPCs +NCsiRNA(group NCsi)

\section{Experiment 3}

Groups

ASTs-OPCs group (group A)

ASTs-OPCs + Cx47siRNA(group Cx47si)

ASTs-OPCs + NCsiRNA(group NCsi)

ASTs-OPCs +Cx47siRNA+exosomes (group Cx47si+)

\section{Experiment 4}

Groups

OPCs group (group $\mathbf{O}$ )

OPCs $+5 \mathrm{ng} / \mathrm{mlChi3l1}$ group (group $5 \mathrm{ch}$ )

OPCs+10ng/mlChi3l1 group (group 10ch)

\section{Experiment 5}

\section{Groups}

OPCs+goat IgG group (group goat)

OPCs+anti Chi3l1 antibody (group Ch)

OPCs+anti Myh9 antibody (group My)

\section{Experiment 6}

Groups

OPCs+10ng/mlChi3l1 group (group 10ch)

OPCs+10ng/mlChi3!1+NCsiRNA (group NCsi2)

OPCs+10ng/mlChi3l1+Myh9 siRNA1 (group NCsil) $-15 \mathrm{c}$

OPCs+10ng/mlChi3l1+Myh9 siRNA2 (group NCsi2)

OPCs+10ng/mlChi3l1+Myh9 siRNA3 (group NCsi3)

$-15 d$

$-15 d$
ASTs-OPCs EDU,flow cytomerty

Co-culture transcriptome sequencing

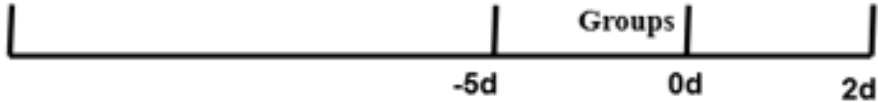

primary culture

ASTs-OPCs

Co-culture

Go,EDU,flow cytomerty Immuno,WB,SIRNA

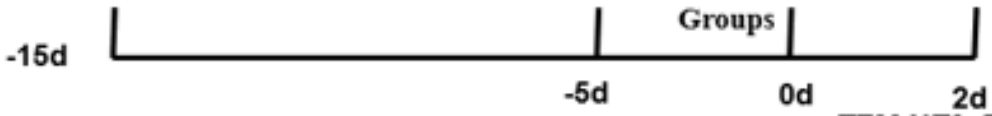

ASTs-OPCs

$\begin{array}{ll}\text { primary culture } & \text { ASTs-OPCs } \\ \text { Co-culture }\end{array}$

TEM,NTA, Go,EDU, flow cytomerty

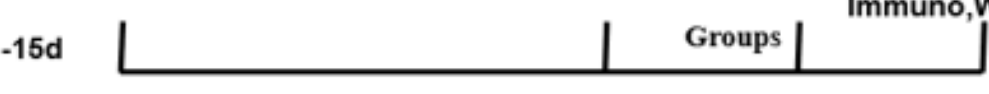

primary culture

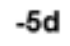

od

2d

OPCs

mono-culture

$-15 d$

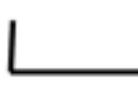

primary culture

OPCs mono-culture groups,Pull-down,
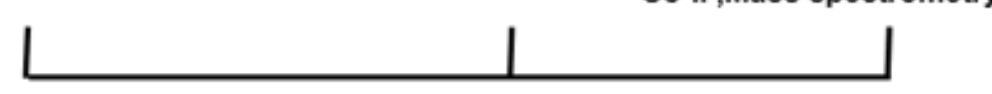

$-5 c$

$-5 d$

mono-culture primary culture
Od EDU,flow cytomerty Immuno,WB, SiRNA,

\section{Figure 1}

Cell group classification and experimental design. 


\section{Experiment 1}

Groups

OPCs group (group $\mathbf{O}$ )

CO-OPCs group (group C)

ASTs-OPCs group (group A)

Experiment 2

Groups

ASTs-OPCs group (group A)

ASTs-OPCs + Cx47siRNA(group Cx47si)

ASTs-OPCs +NCsiRNA(group NCsi)

\section{Experiment 3}

Groups

ASTs-OPCs group (group A)

ASTs-OPCs + Cx47siRNA(group Cx47si)

ASTs-OPCs + NCsiRNA(group NCsi)

ASTs-OPCs +Cx47siRNA+exosomes (group Cx47si+)

\section{Experiment 4}

Groups

OPCs group (group $\mathbf{O}$ )

OPCs $+5 \mathrm{ng} / \mathrm{mlChi} 311$ group (group $5 \mathrm{ch}$ )

OPCs+10ng/mIChi3l1 group (group 10ch)

\section{Experiment 5}

\section{Groups}

OPCs+goat IgG group (group goat)

OPCs+anti Chi3l1 antibody (group $\mathrm{Ch}$ )

OPCs+anti Myh9 antibody (group My)

\section{Experiment 6}

Groups

OPCs+10ng/mlChi3l1 group (group 10ch)

OPCs+10ng/mlChi3l1+NCsiRNA (group NCsi2)

OPCs+10ng/mlChi3l1+Myh9 siRNA1 (group NCsil) $-15 \mathrm{c}$

OPCs+10ng/mlChi3l1+Myh9 siRNA2 (group NCsi2)

OPCs+10ng/mlChi3l1+Myh9 siRNA3 (group NCsi3)

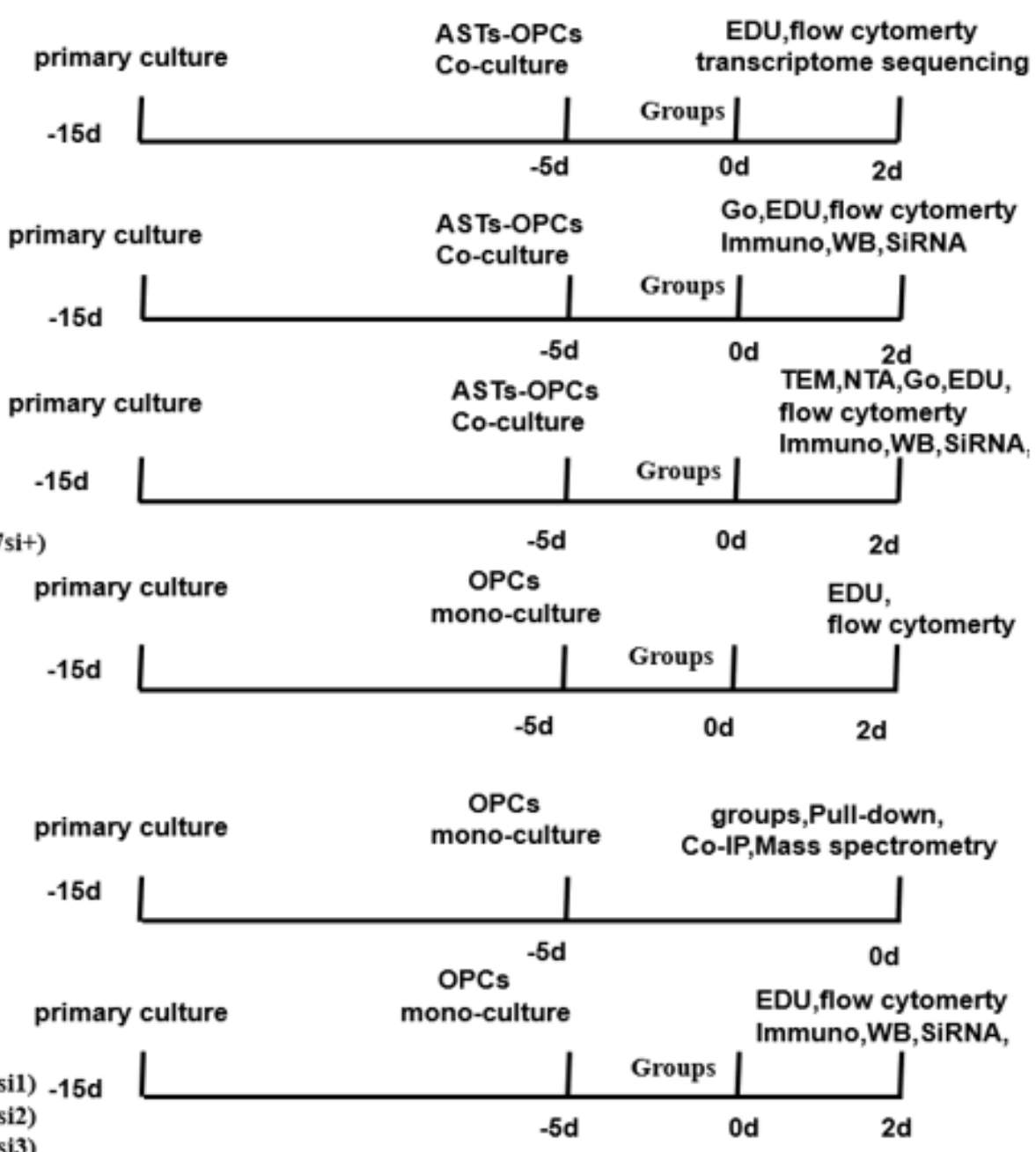

\section{Figure 1}

Cell group classification and experimental design. 


\section{Experiment 1}

Groups

OPCs group (group $\mathbf{O}$ )

CO-OPCs group (group C)

ASTs-OPCs group (group A)

Experiment 2

Groups

ASTs-OPCs group (group A)

ASTs-OPCs + Cx47siRNA(group Cx47si)

ASTs-OPCs +NCsiRNA(group NCsi)

\section{Experiment 3}

Groups

ASTs-OPCs group (group A)

ASTs-OPCs + Cx47siRNA(group Cx47si)

ASTs-OPCs + NCsiRNA(group NCsi)

ASTs-OPCs +Cx47siRNA+exosomes (group Cx47si+)

\section{Experiment 4}

Groups

OPCs group (group $\mathbf{O}$ )

OPCs $+5 \mathrm{ng} / \mathrm{mlChi3l1}$ group (group $5 \mathrm{ch}$ )

OPCs+10ng/mlChi3l1 group (group 10ch)

\section{Experiment 5}

\section{Groups}

OPCs+goat IgG group (group goat)

OPCs+anti Chi3l1 antibody (group Ch)

OPCs+anti Myh9 antibody (group My)

\section{Experiment 6}

Groups

OPCs+10ng/mlChi3l1 group (group 10ch)

OPCs+10ng/mlChi3!1+NCsiRNA (group NCsi2)

OPCs+10ng/mlChi3l1+Myh9 siRNA1 (group NCsil) $-15 \mathrm{c}$

OPCs+10ng/mlChi3l1+Myh9 siRNA2 (group NCsi2)

OPCs+10ng/mlChi3l1+Myh9 siRNA3 (group NCsi3)

$-15 d$

$-15 d$
ASTs-OPCs EDU,flow cytomerty

Co-culture transcriptome sequencing

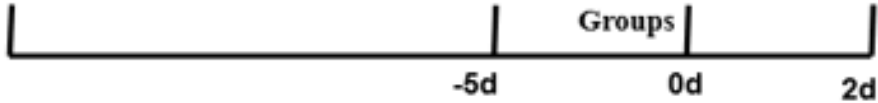

primary culture

ASTs-OPCs

Co-culture

Go,EDU,flow cytomerty Immuno,WB,SIRNA

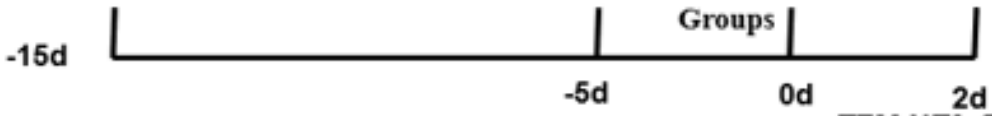

ASTs-OPCs

$\begin{array}{ll}\text { primary culture } & \text { ASTs-OPCs } \\ \text { Co-culture }\end{array}$

TEM,NTA, Go,EDU, flow cytomerty

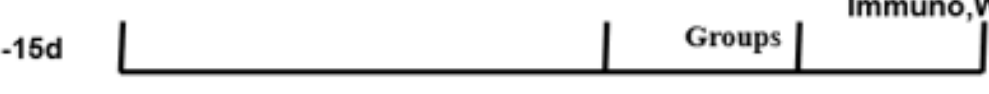

primary culture

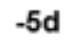

od

2d

OPCs

mono-culture

$-15 d$

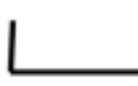

primary culture

OPCs mono-culture groups,Pull-down,
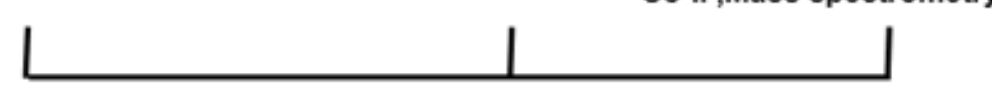

$-5 c$

$-5 d$

mono-culture primary culture
Od EDU,flow cytomerty Immuno,WB, SiRNA,

\section{Figure 1}

Cell group classification and experimental design. 
A

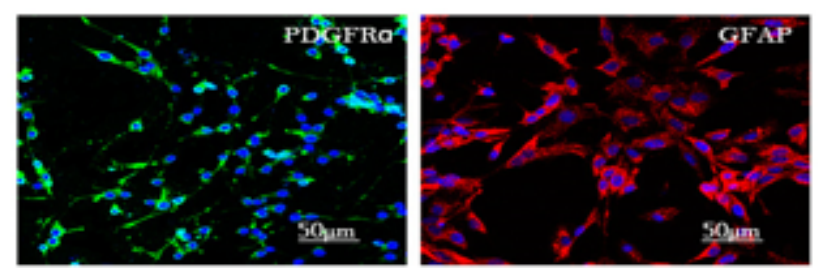

B
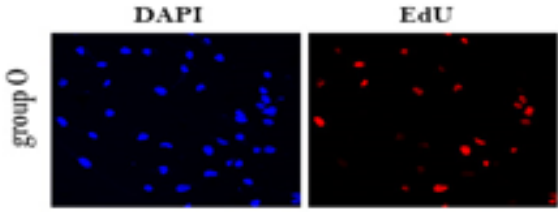

merge
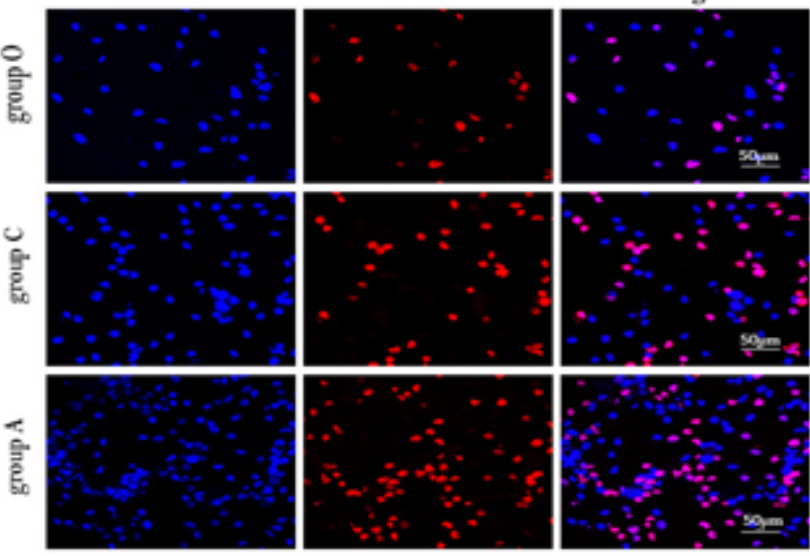

C
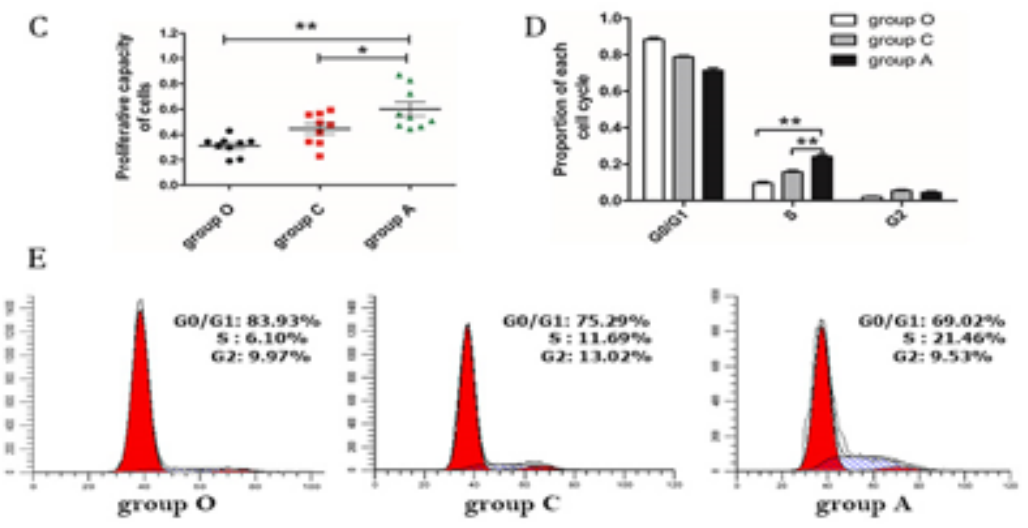

Figure 2

Direct co-culture of ASTs and OPCs promotes the proliferation of OPCs. 
A

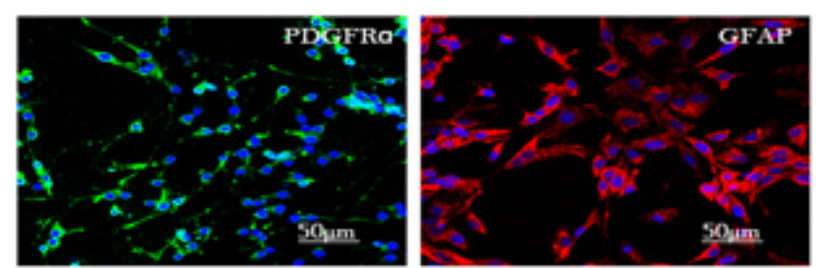

B
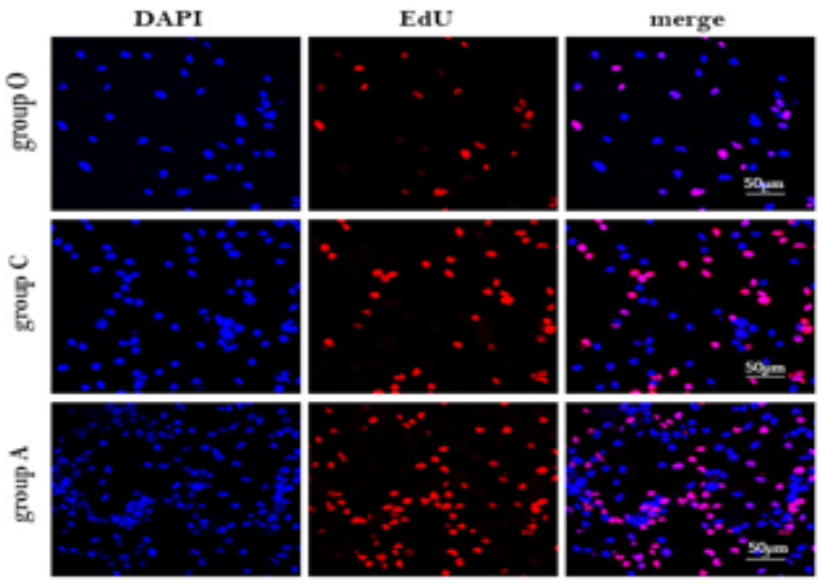

C
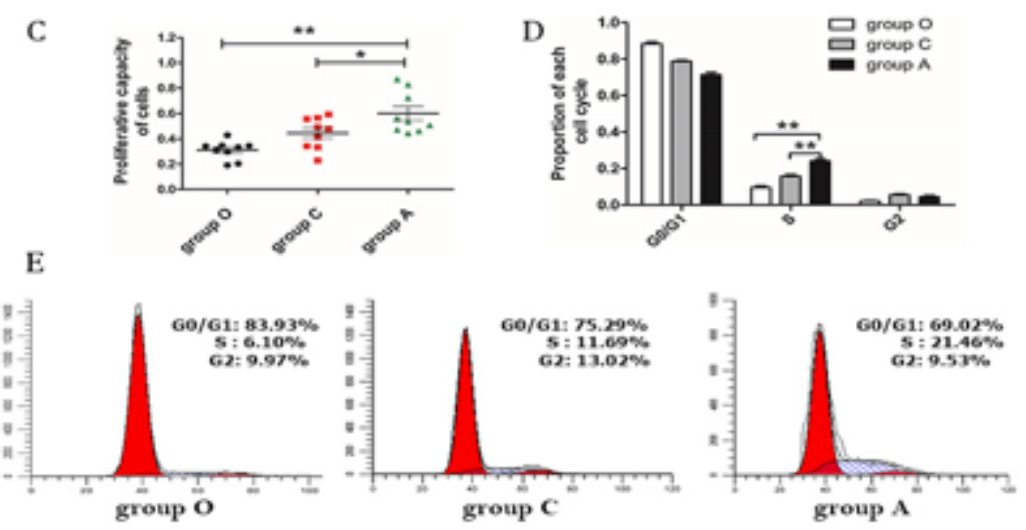

Figure 2

Direct co-culture of ASTs and OPCs promotes the proliferation of OPCs. 
A

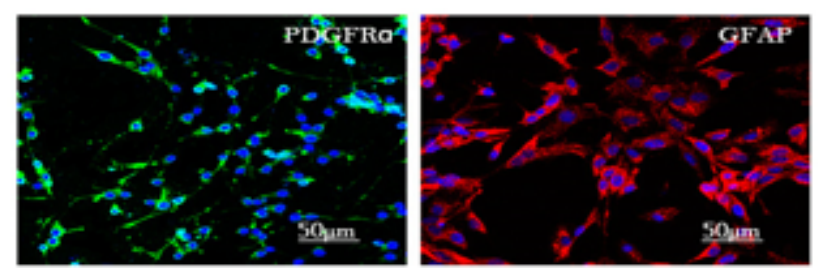

B
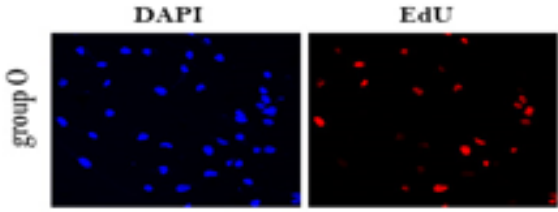

merge
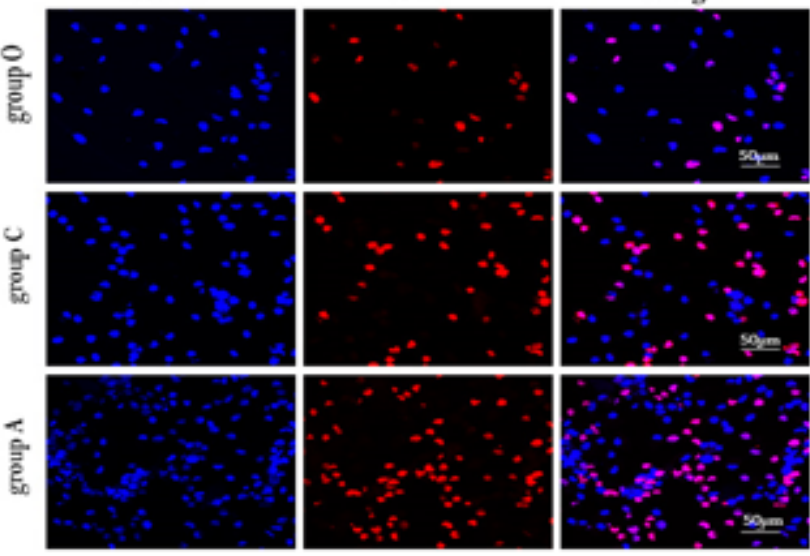

C
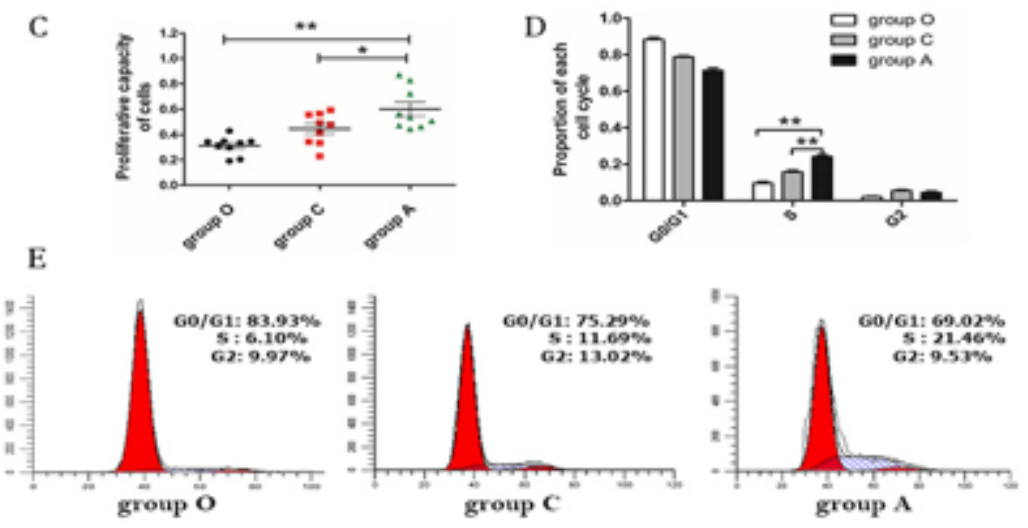

Figure 2

Direct co-culture of ASTs and OPCs promotes the proliferation of OPCs. 
A

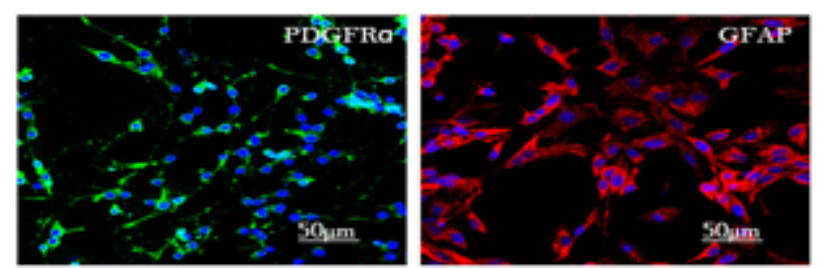

B
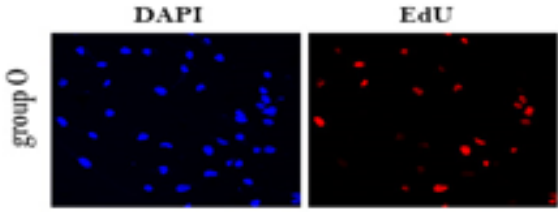

merge
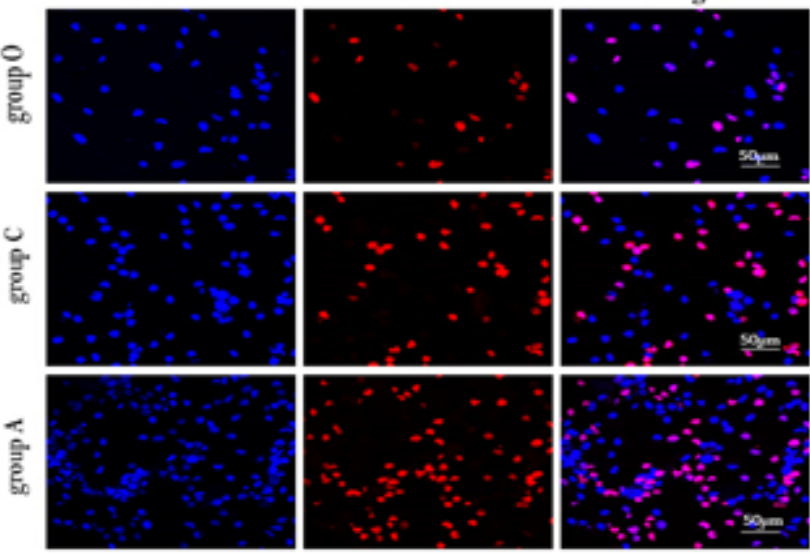

C
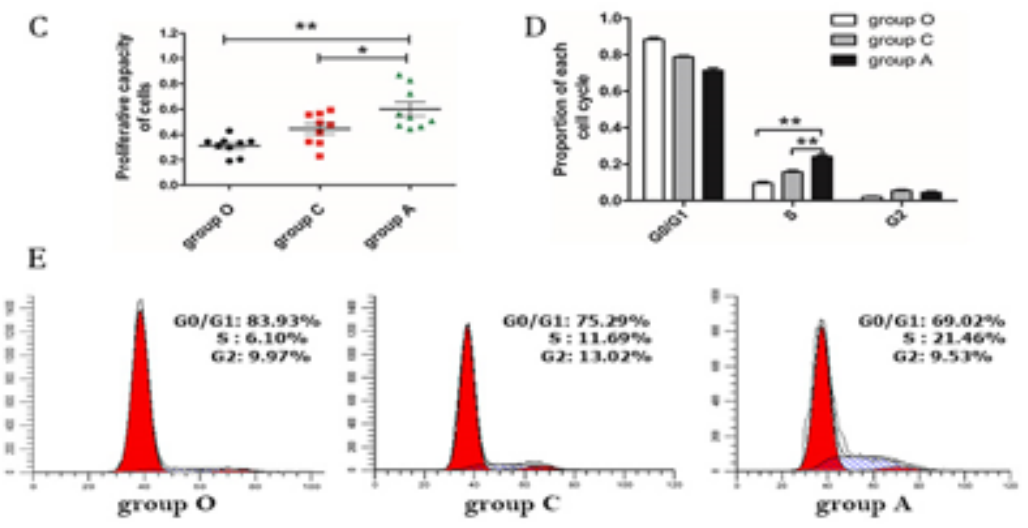

Figure 2

Direct co-culture of ASTs and OPCs promotes the proliferation of OPCs. 
A

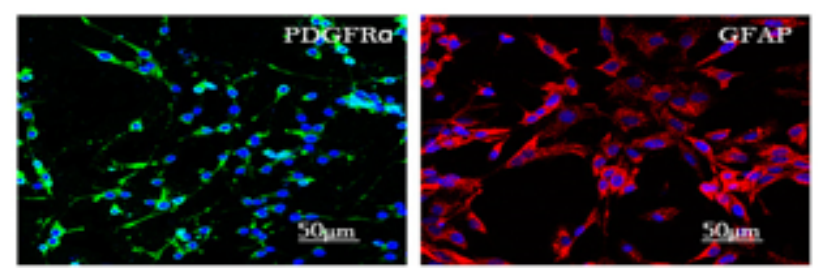

B
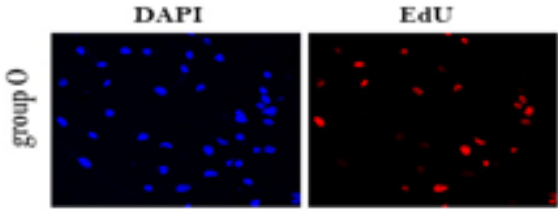

merge
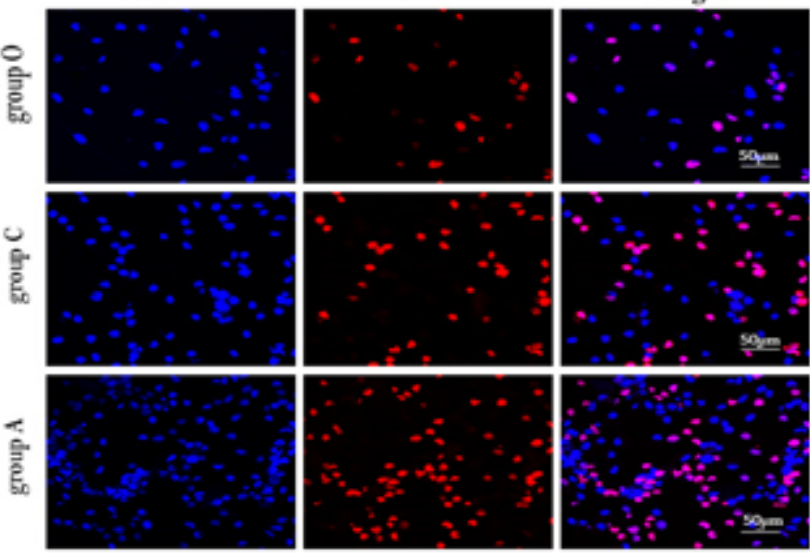

C
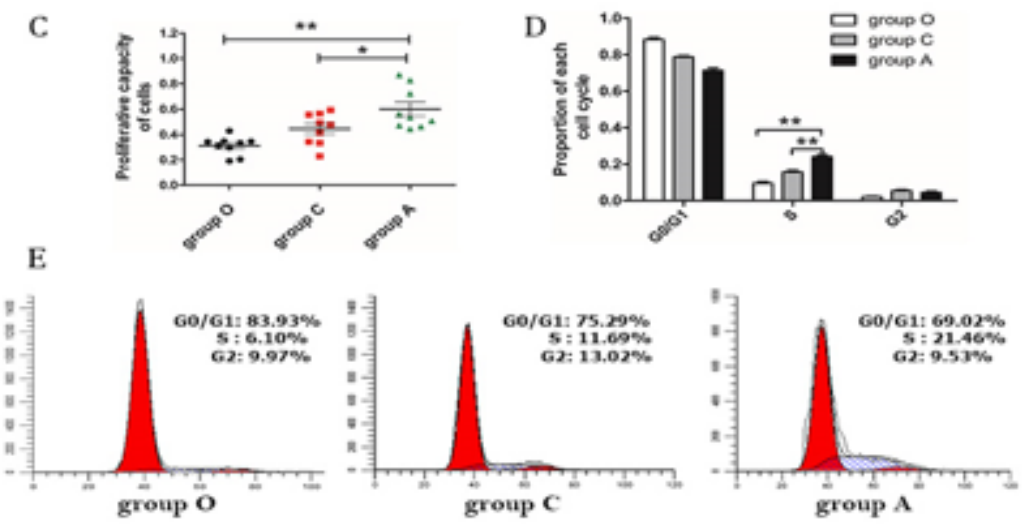

Figure 2

Direct co-culture of ASTs and OPCs promotes the proliferation of OPCs. 
A

B

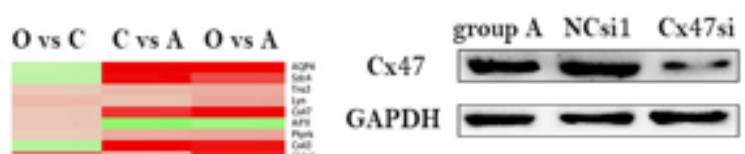

C

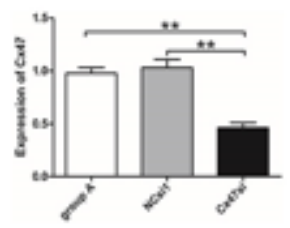

D

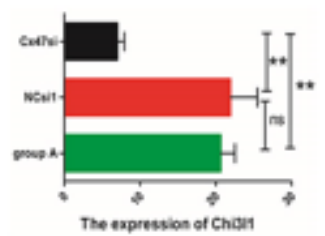

E

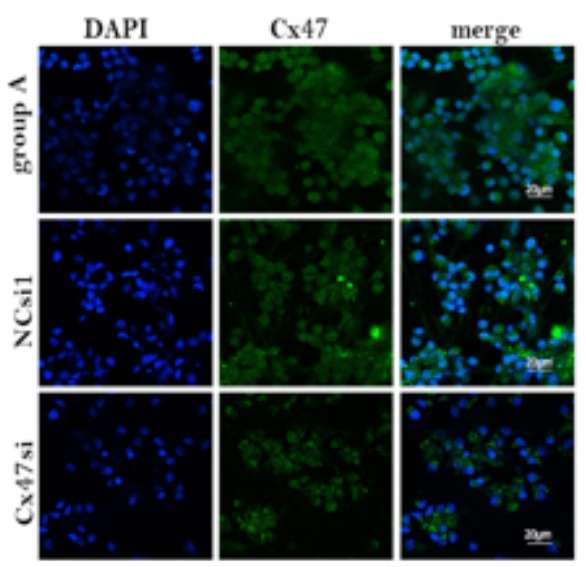

G

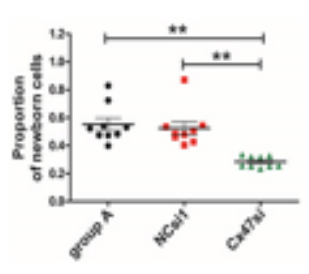

H

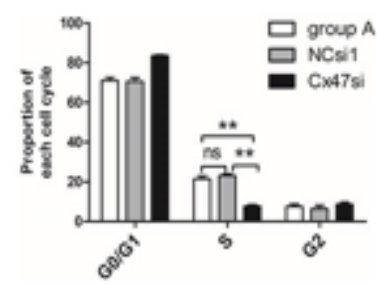

I
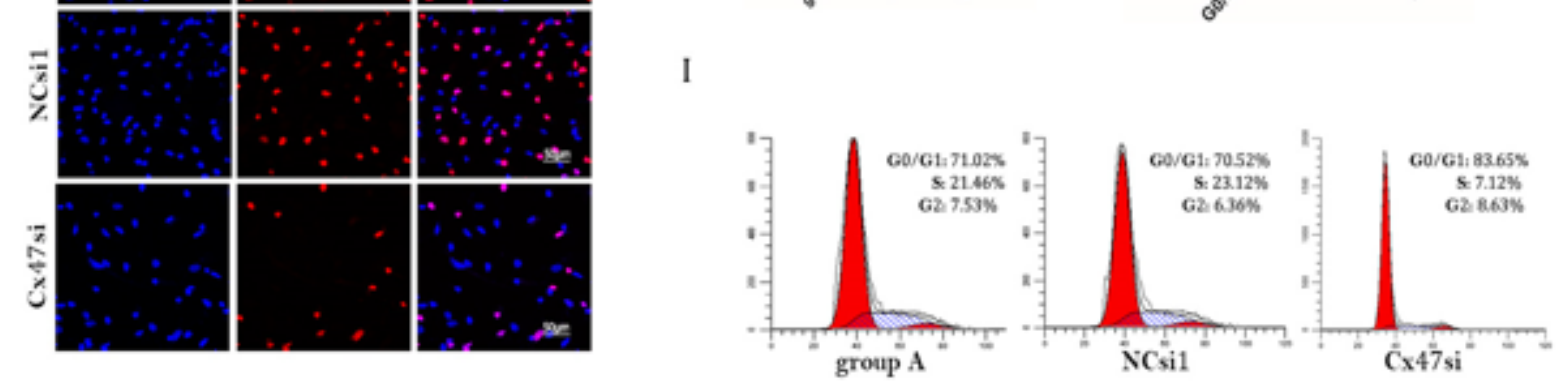

Figure 3

Interfere with Cx47 under ASTs and OPCs co-culture conditions, the proliferative of OPCs decreased. 
A

B

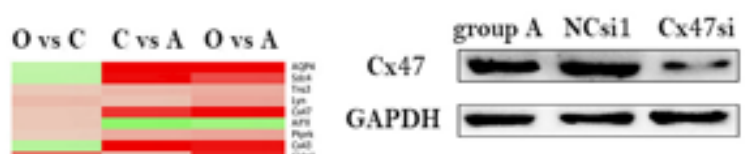

C

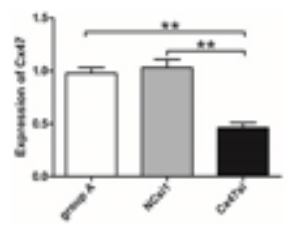

D

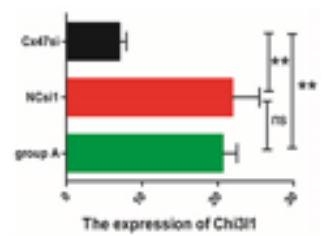

E

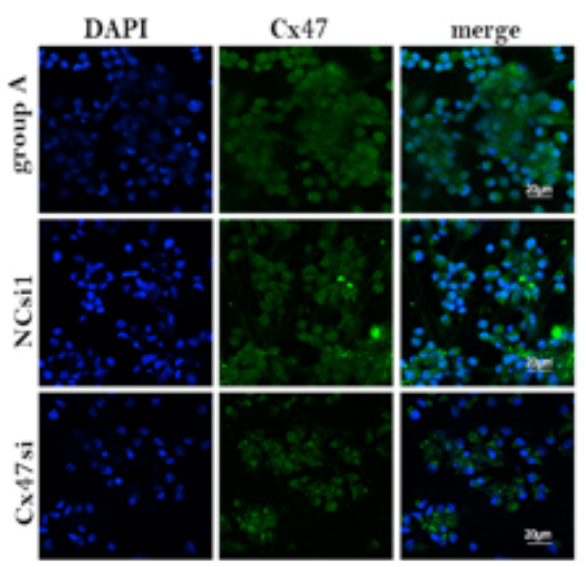

G

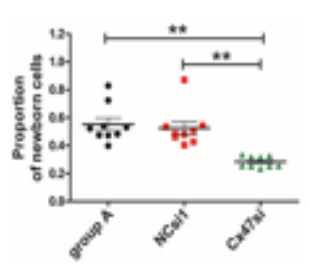

H

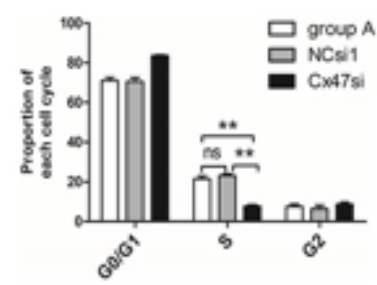

I
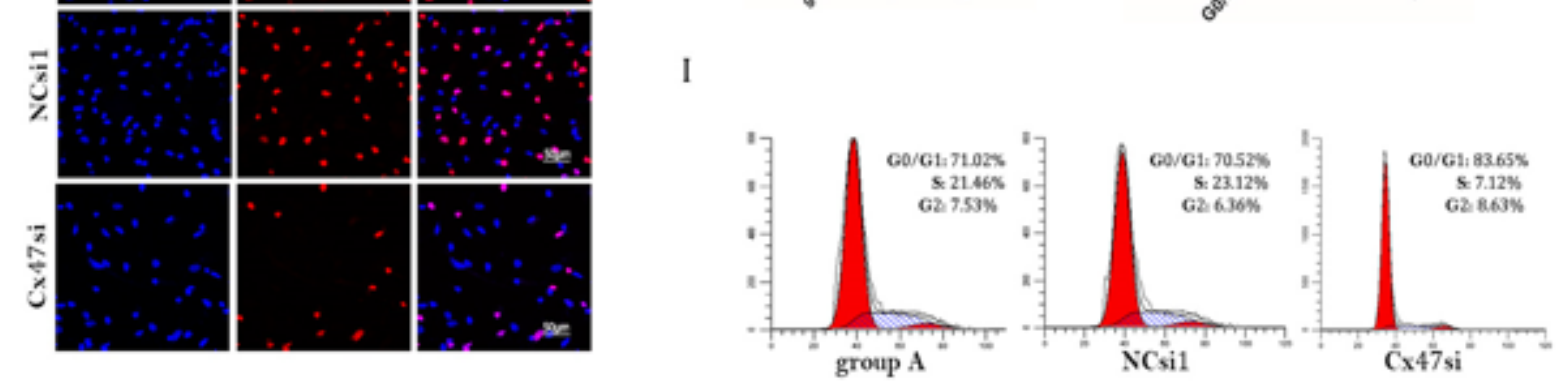

Figure 3

Interfere with Cx47 under ASTs and OPCs co-culture conditions, the proliferative of OPCs decreased. 
A

B

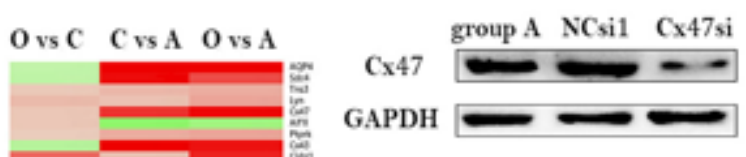

C

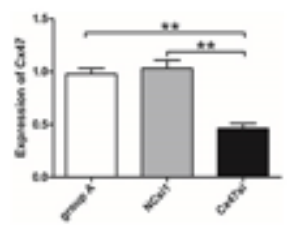

D

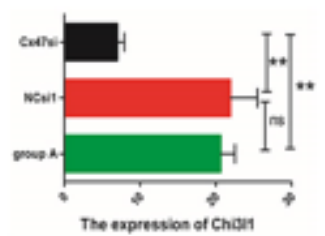

E

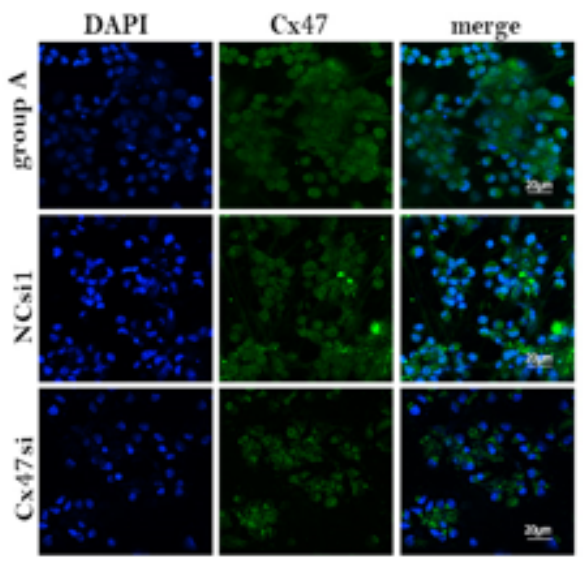

G

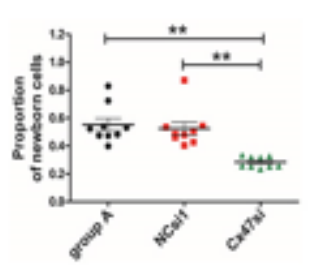

H

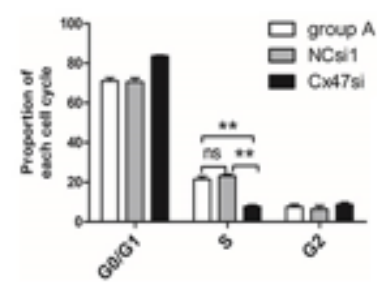

I
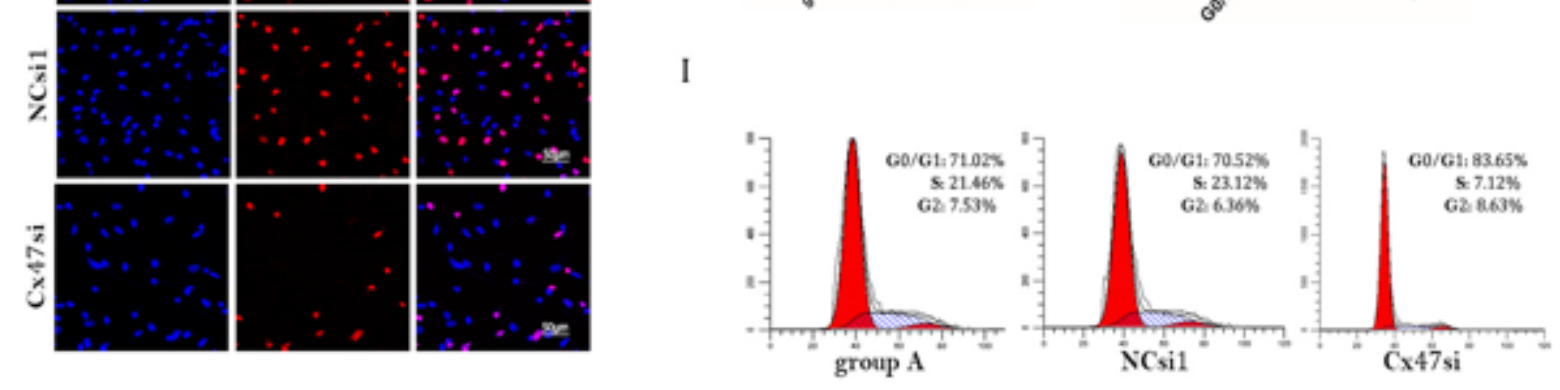

Figure 3

Interfere with Cx47 under ASTs and OPCs co-culture conditions, the proliferative of OPCs decreased. 
A

B

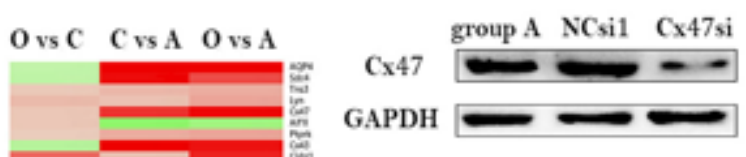

C

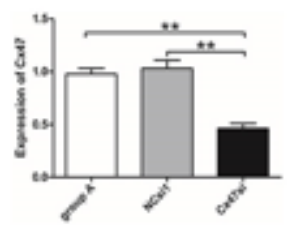

D

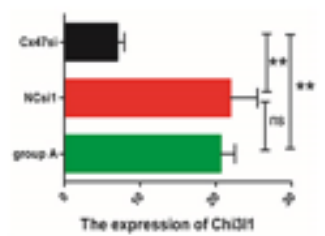

E

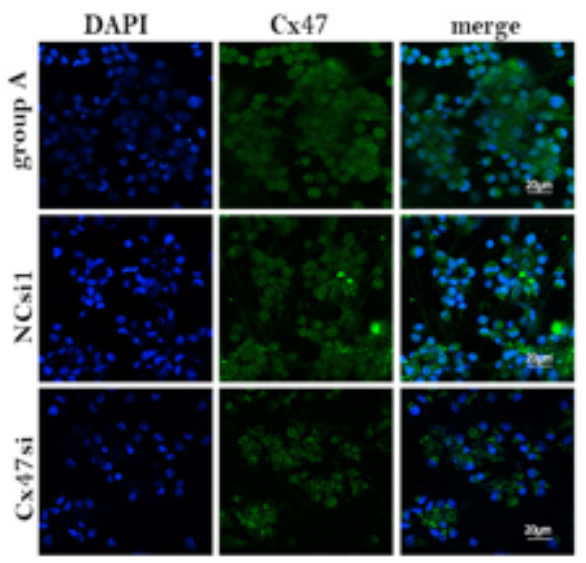

G

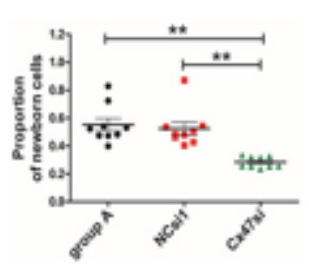

H

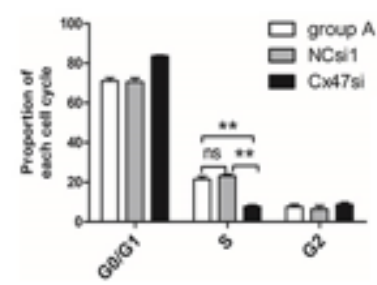

I
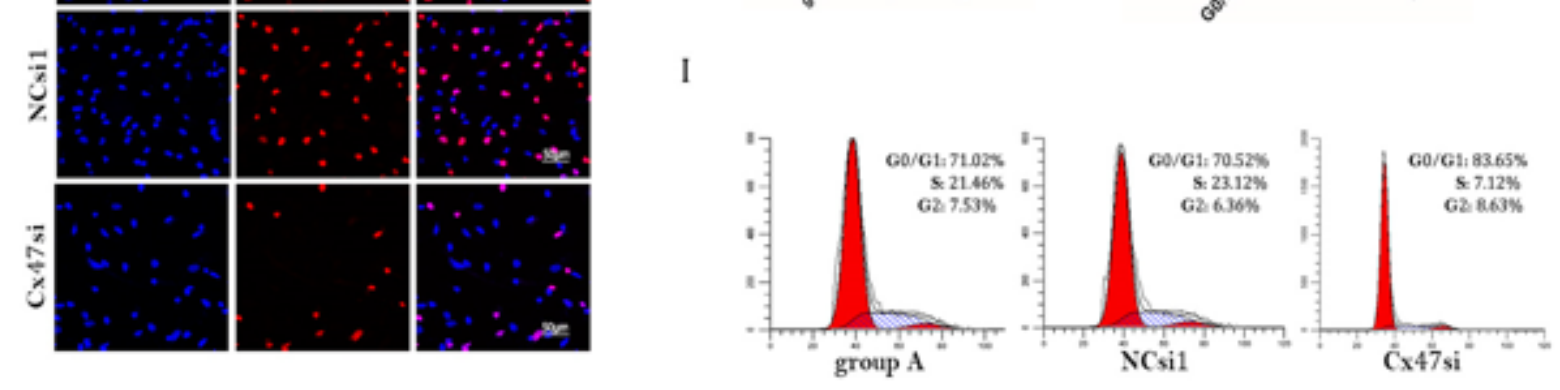

Figure 3

Interfere with Cx47 under ASTs and OPCs co-culture conditions, the proliferative of OPCs decreased. 
A

B

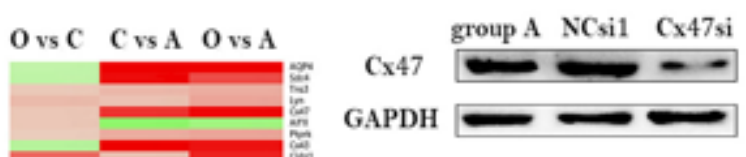

C

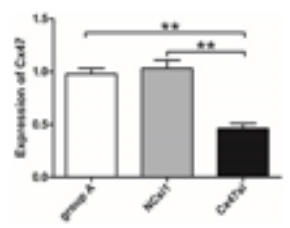

D

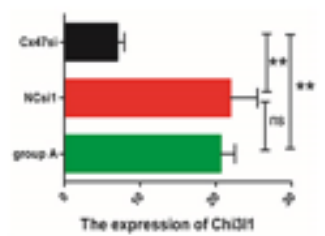

E

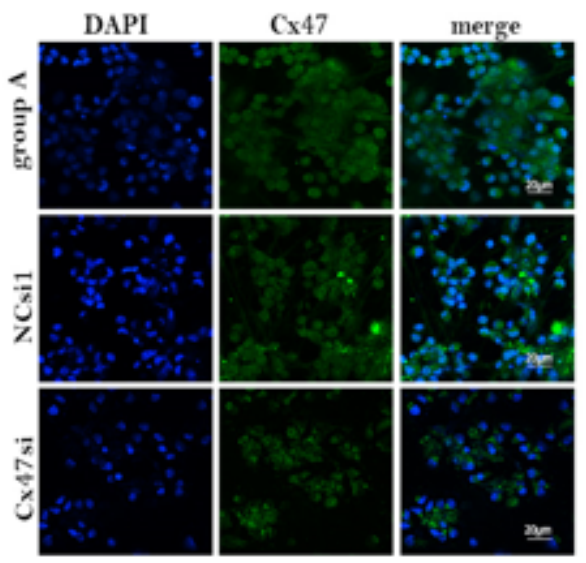

G

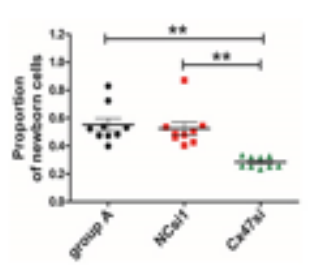

H

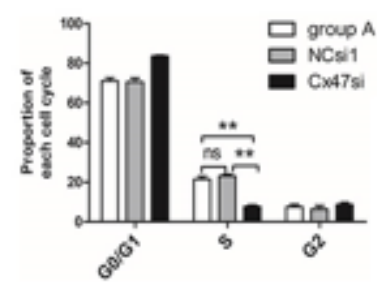

I
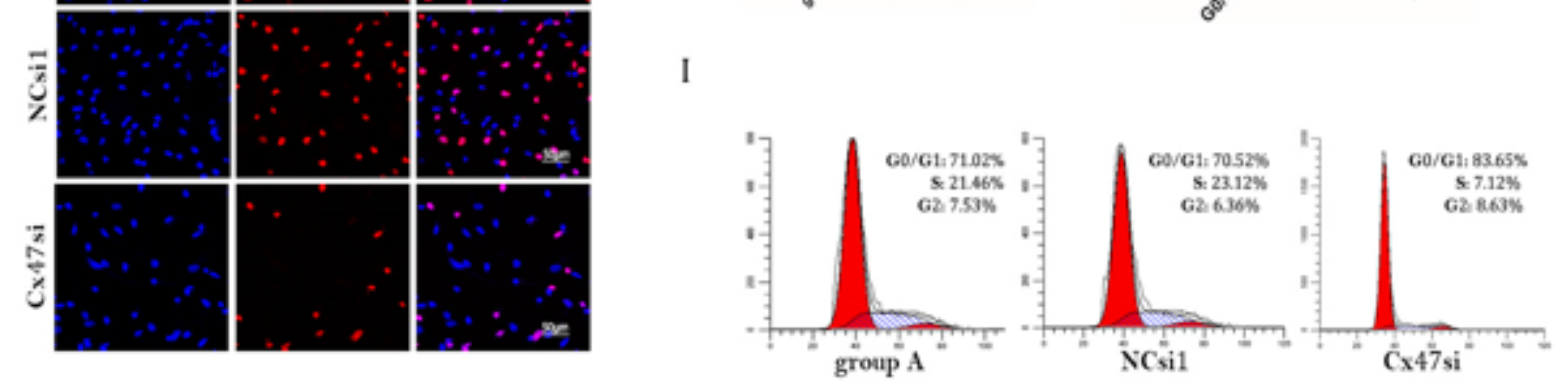

Figure 3

Interfere with Cx47 under ASTs and OPCs co-culture conditions, the proliferative of OPCs decreased. 


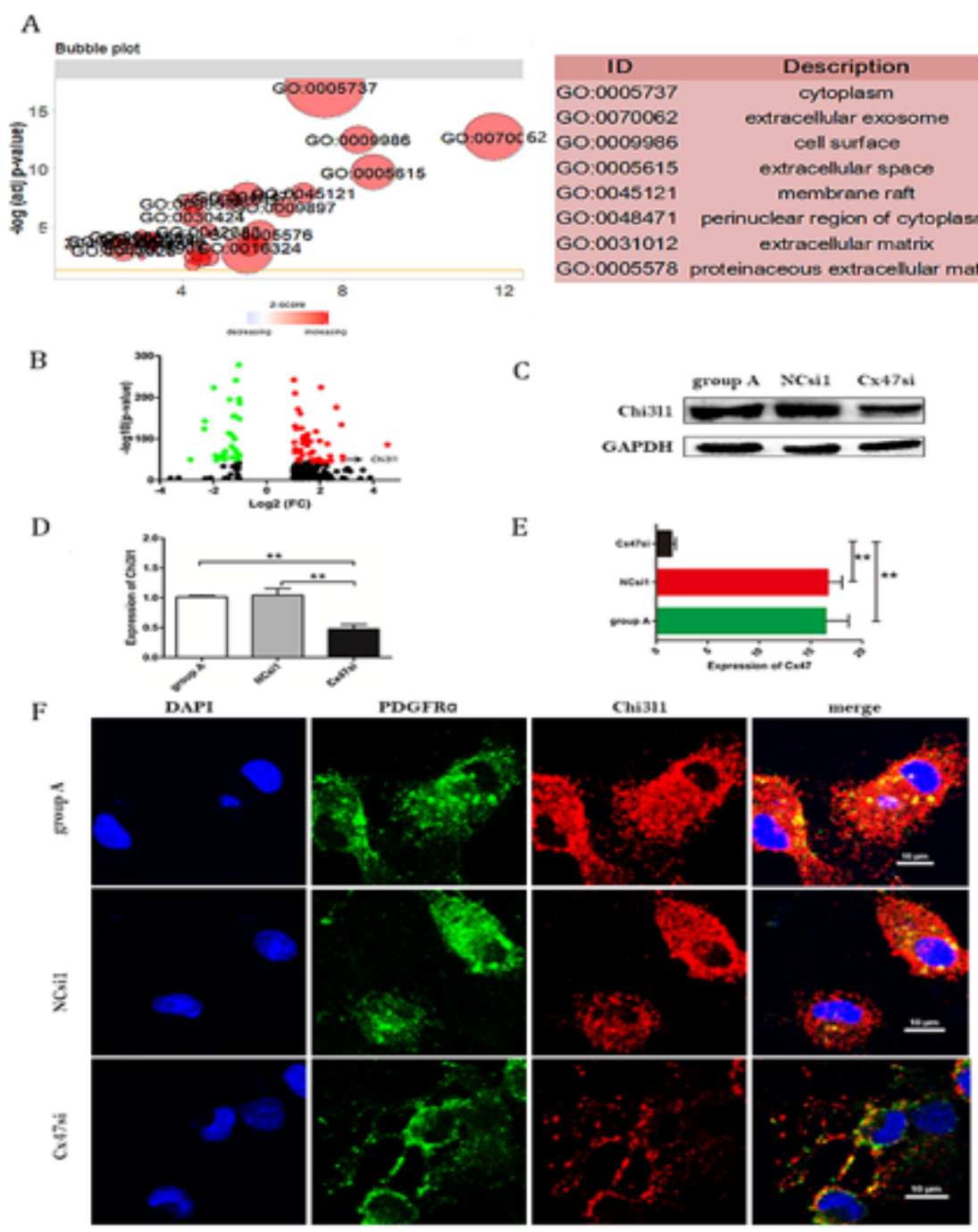

Figure 4

Chi3l1 expression in OPCs decreases after Cx47 siRNA interference under ASTs-OPCs co-culture conditions. 


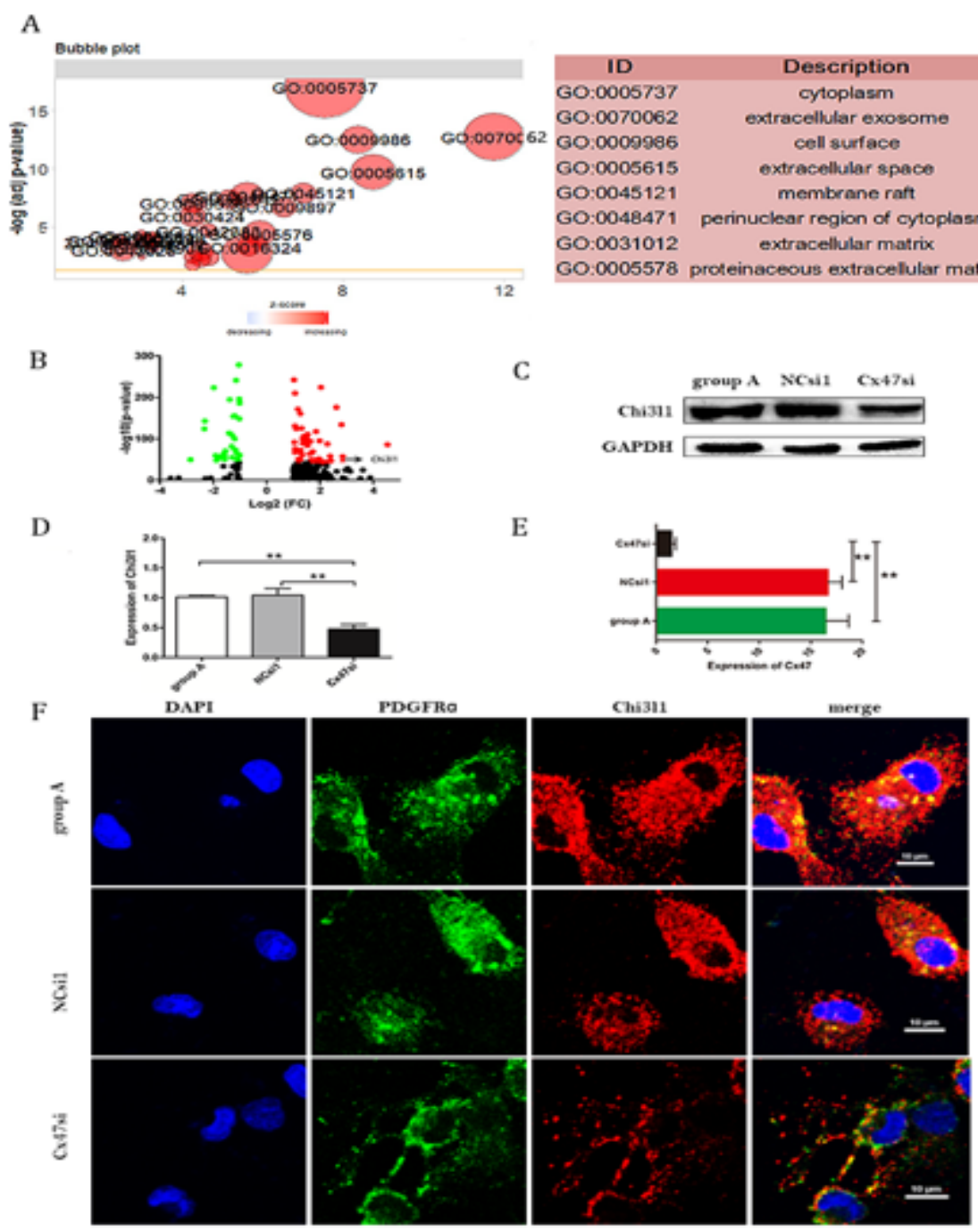

Figure 4

Chi3l1 expression in OPCs decreases after Cx47 siRNA interference under ASTs-OPCs co-culture conditions. 


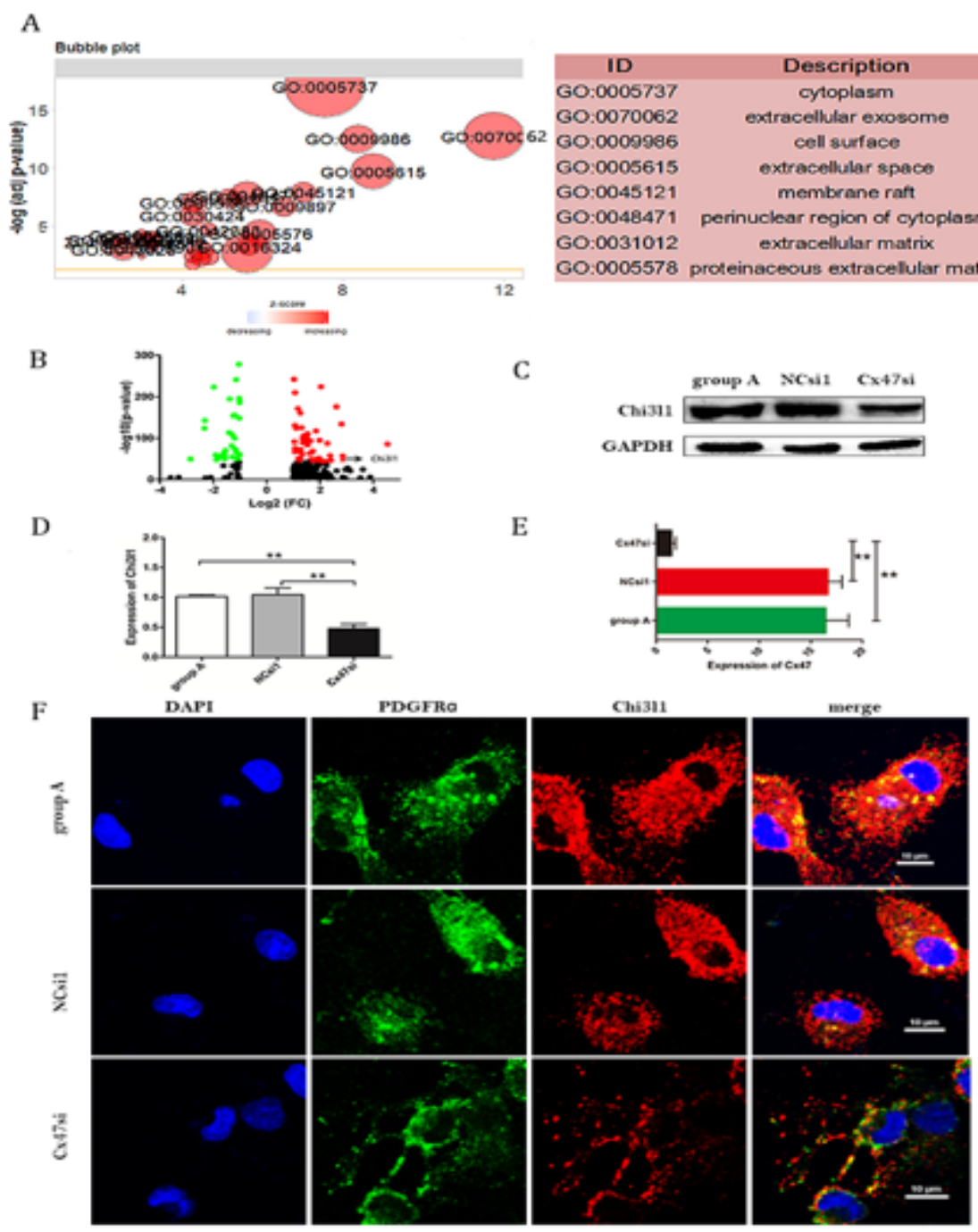

Figure 4

Chi3l1 expression in OPCs decreases after Cx47 siRNA interference under ASTs-OPCs co-culture conditions. 


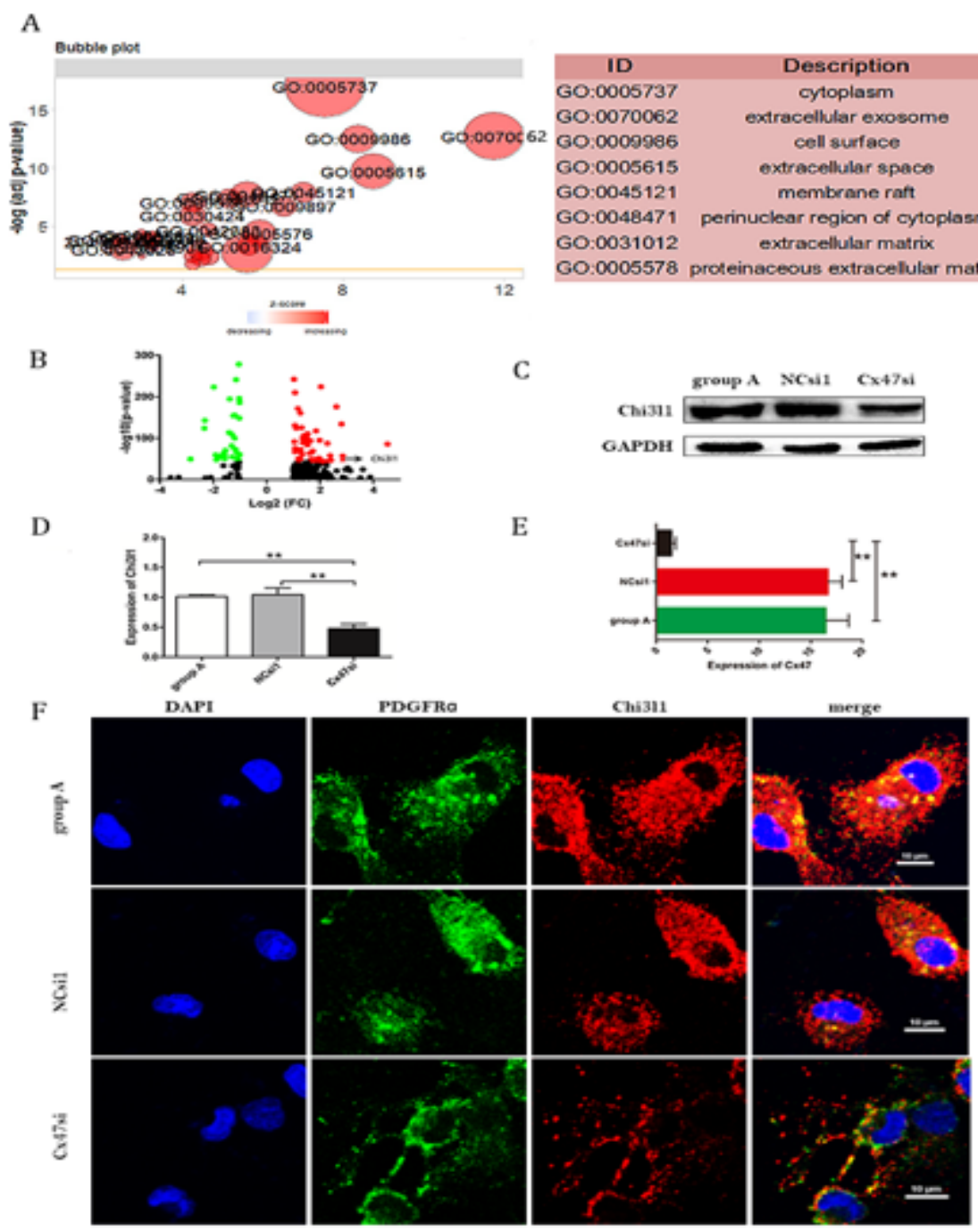

Figure 4

Chi3l1 expression in OPCs decreases after Cx47 siRNA interference under ASTs-OPCs co-culture conditions. 


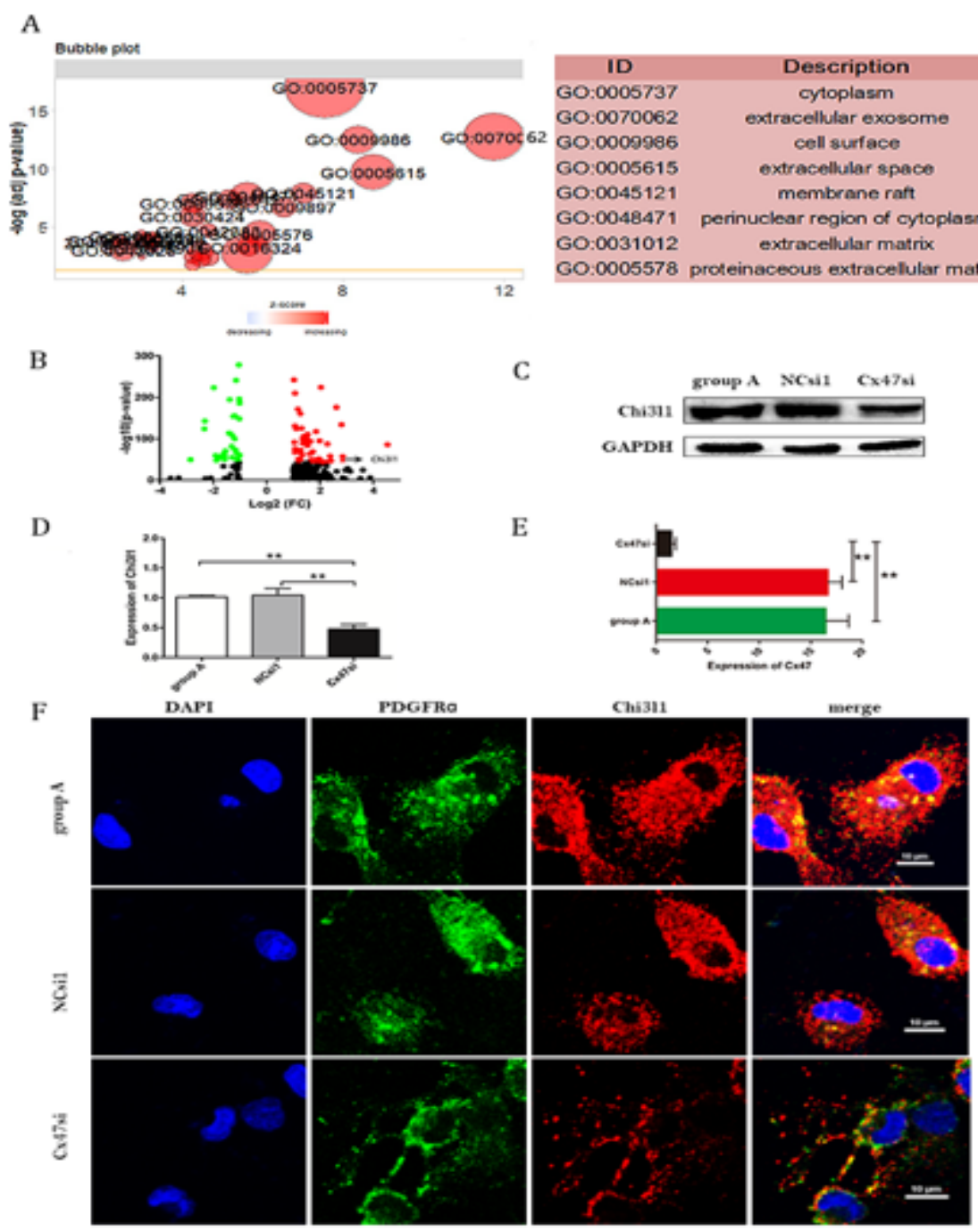

Figure 4

Chi3l1 expression in OPCs decreases after Cx47 siRNA interference under ASTs-OPCs co-culture conditions. 
A

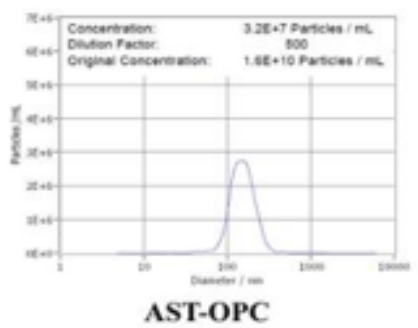

C

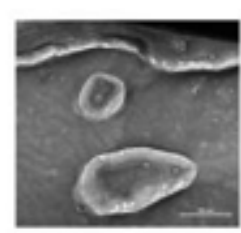

I

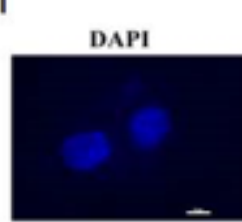

D

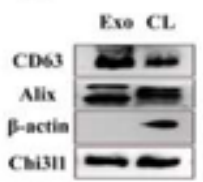

cisn

J

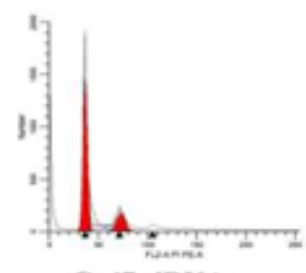

Cx47 SiRNA

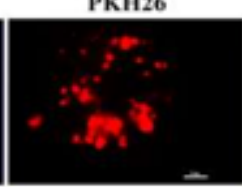

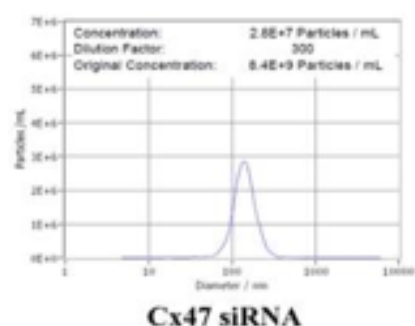

B

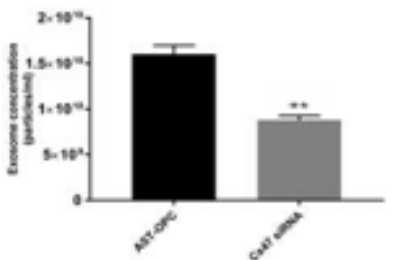

E

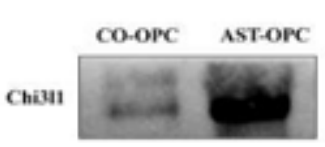

G

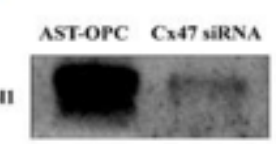

F

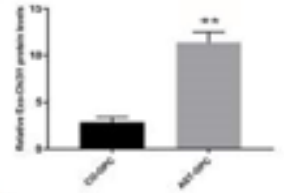

$\mathrm{H}$

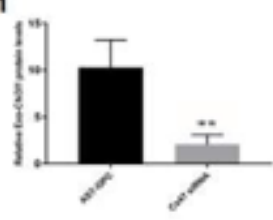

Merge
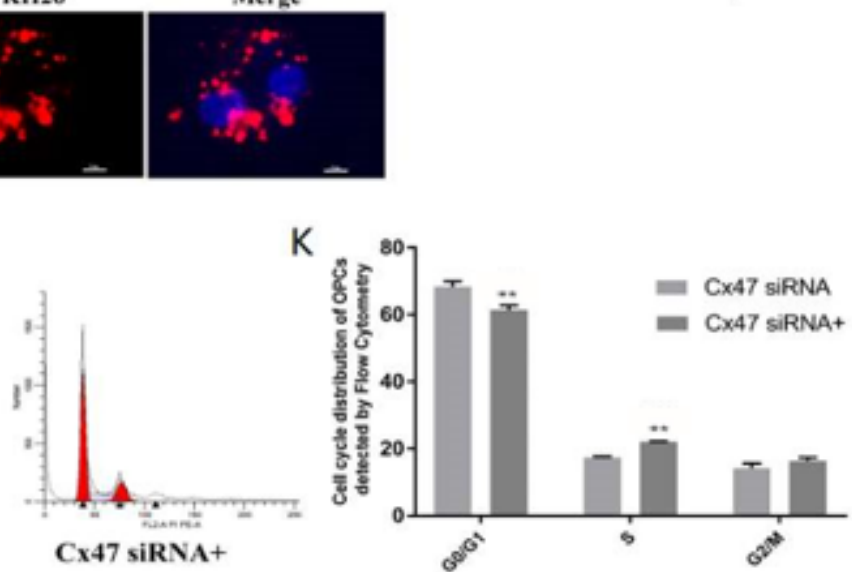

Figure 5

Cx47 regulates the expression of Chi3/1 secretion in exosome to promotes OPCs proliferation. 
A

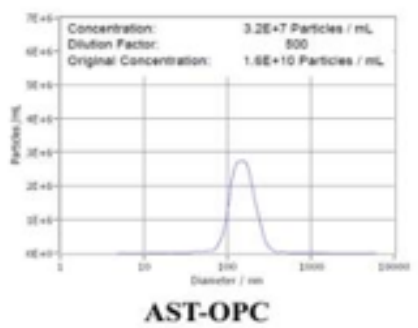

C

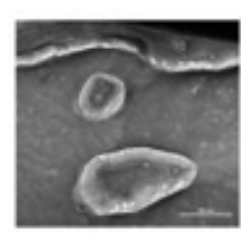

I

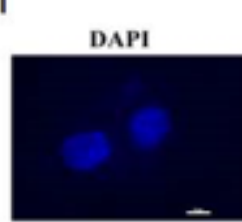

D

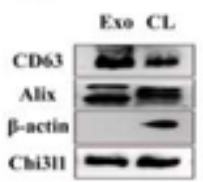

cisn

J

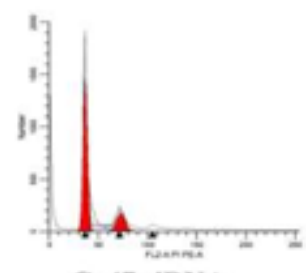

Cx47 SiRNA

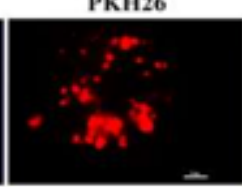

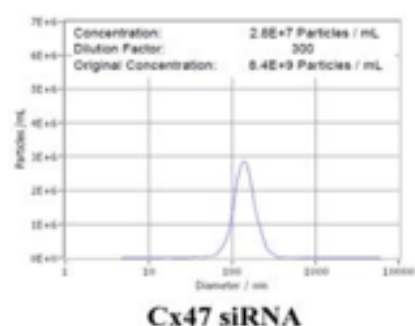

B

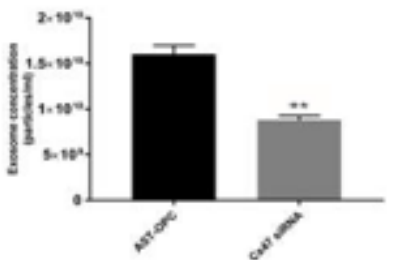

E

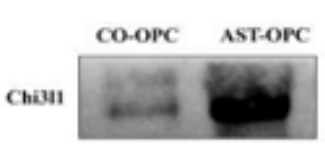

G

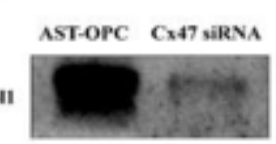

F

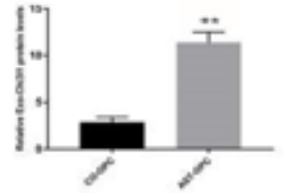

$\mathrm{H}$

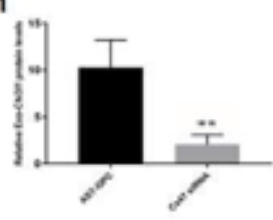

Merge
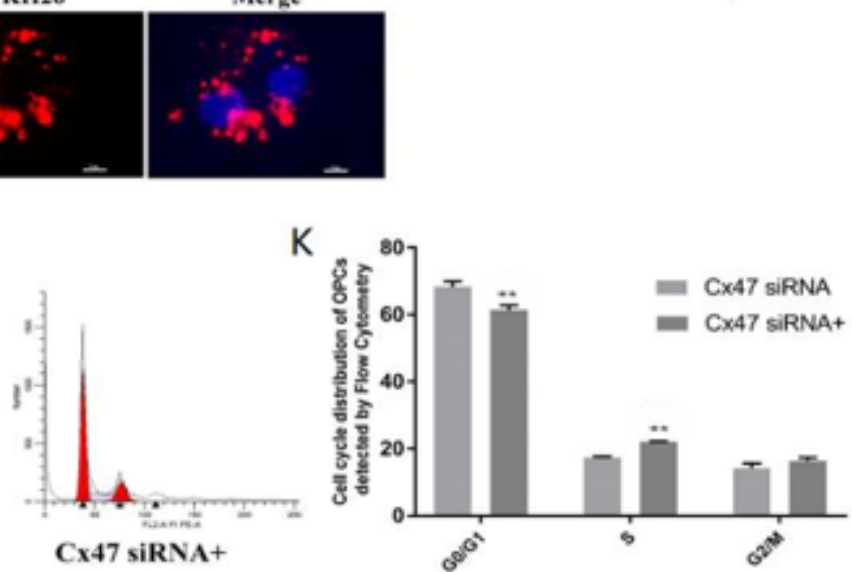

Figure 5

Cx47 regulates the expression of Chi3/1 secretion in exosome to promotes OPCs proliferation. 
A

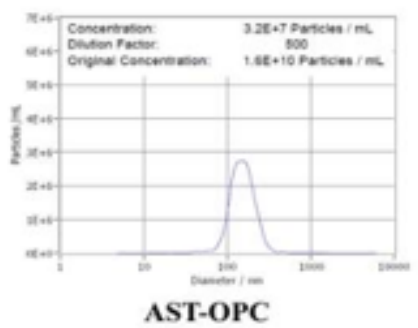

C

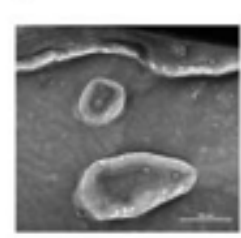

I

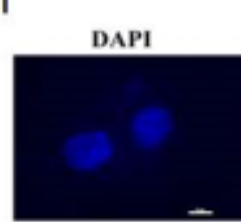

D

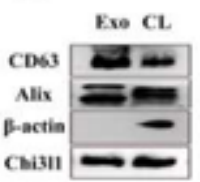

cisn

J

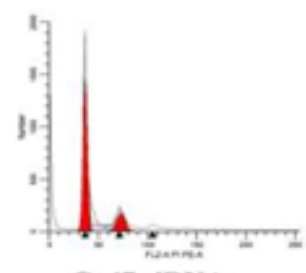

Cx47 SiRNA

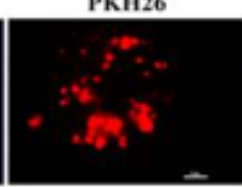

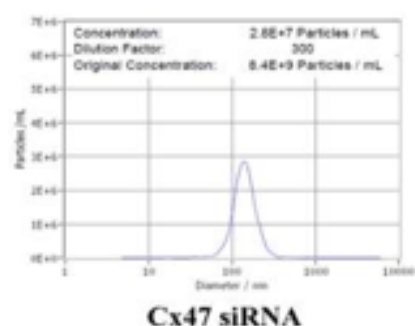

B

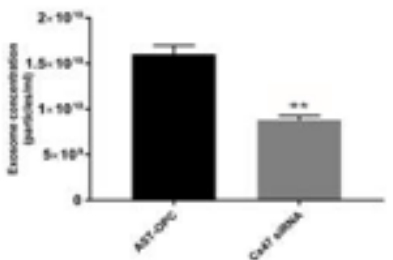

E

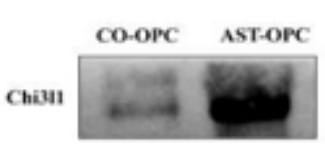

G

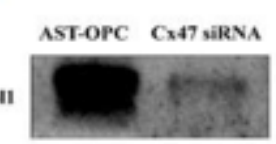

F

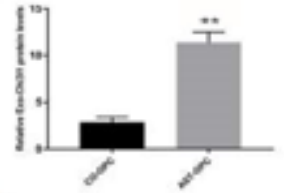

$\mathrm{H}$

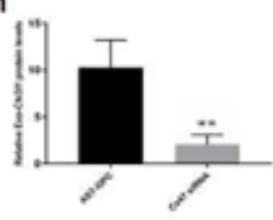

Merge
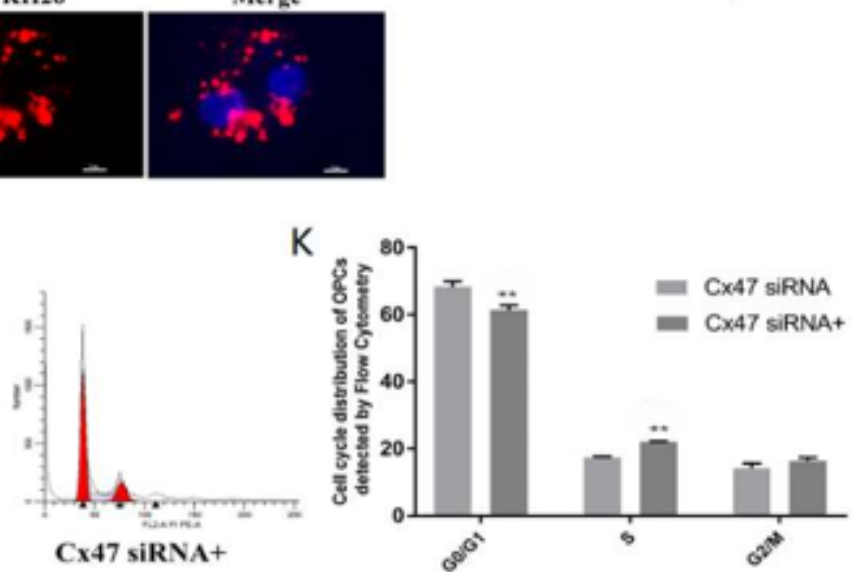

Figure 5

Cx47 regulates the expression of Chi3/1 secretion in exosome to promotes OPCs proliferation. 
A

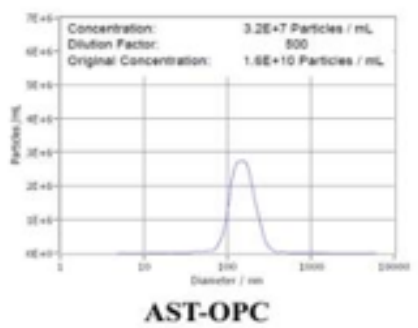

C

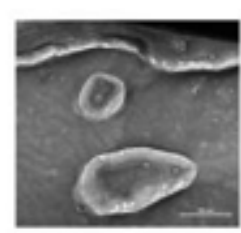

I

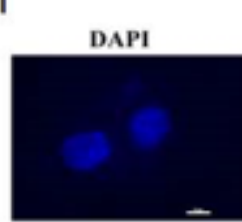

D

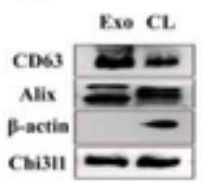

cisn

J

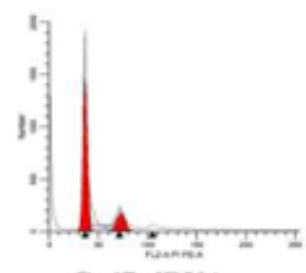

Cx47 SiRNA

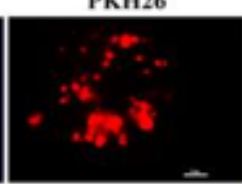

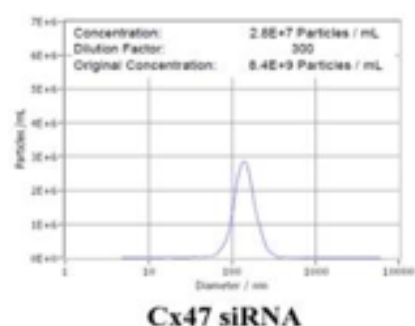

B

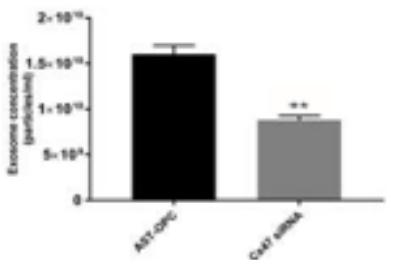

E

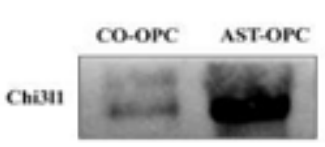

G

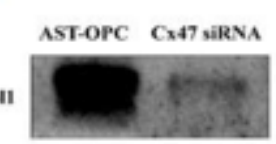

F

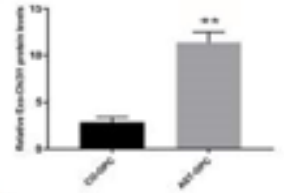

$\mathrm{H}$

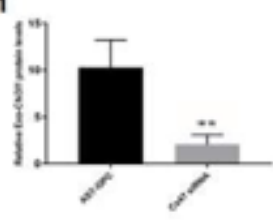

Merge
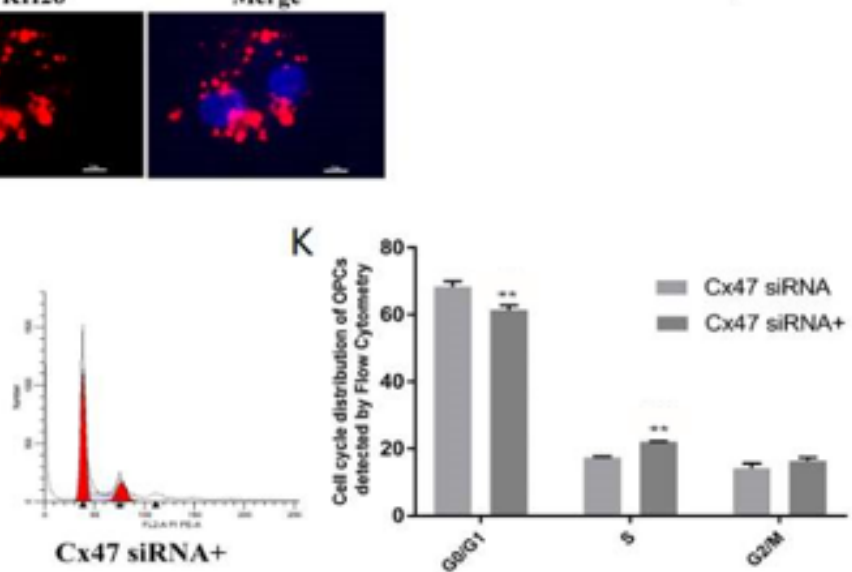

Figure 5

Cx47 regulates the expression of Chi3/1 secretion in exosome to promotes OPCs proliferation. 
A

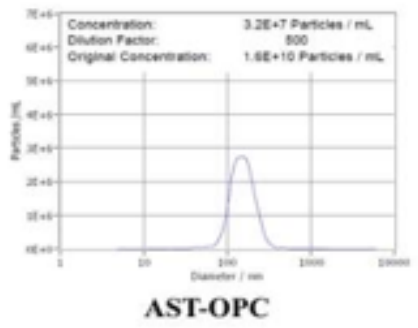

C

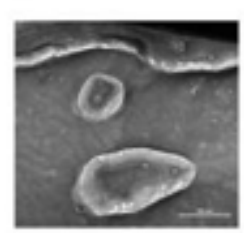

D

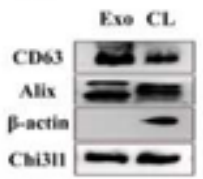

cisn

I

DAPI

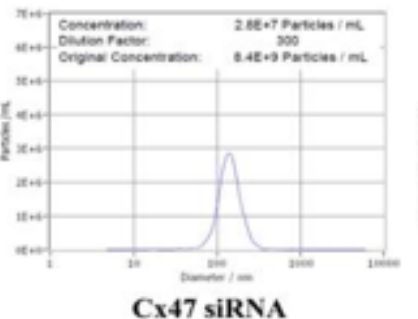

B

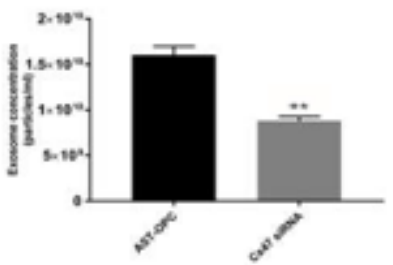

E

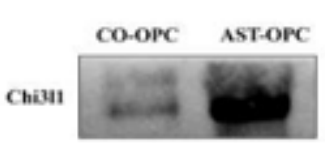

G

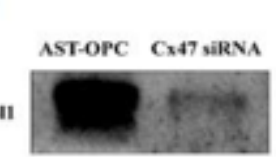

Chisı

Merge

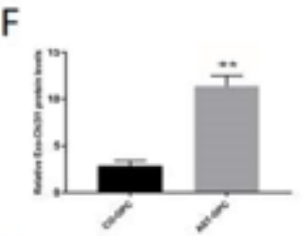

$\mathrm{H}$

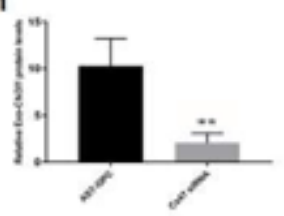

PKH26
J

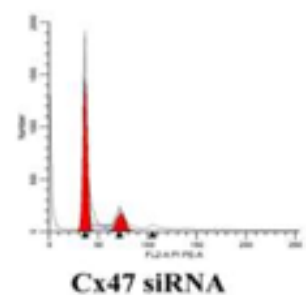

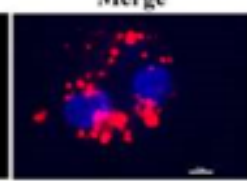

K
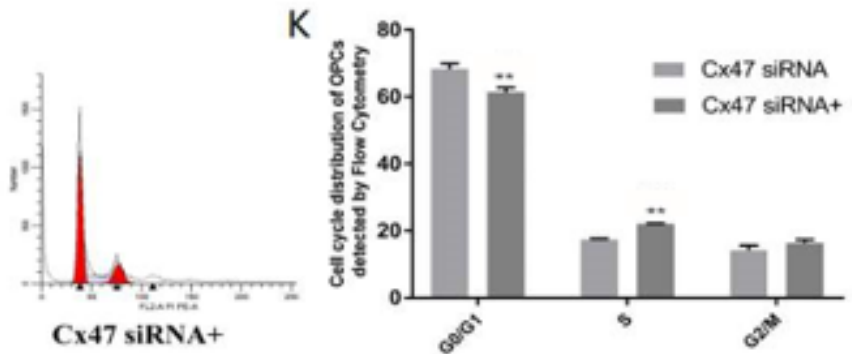

Figure 5

Cx47 regulates the expression of Chi3I1 secretion in exosome to promotes OPCs proliferation. 
A
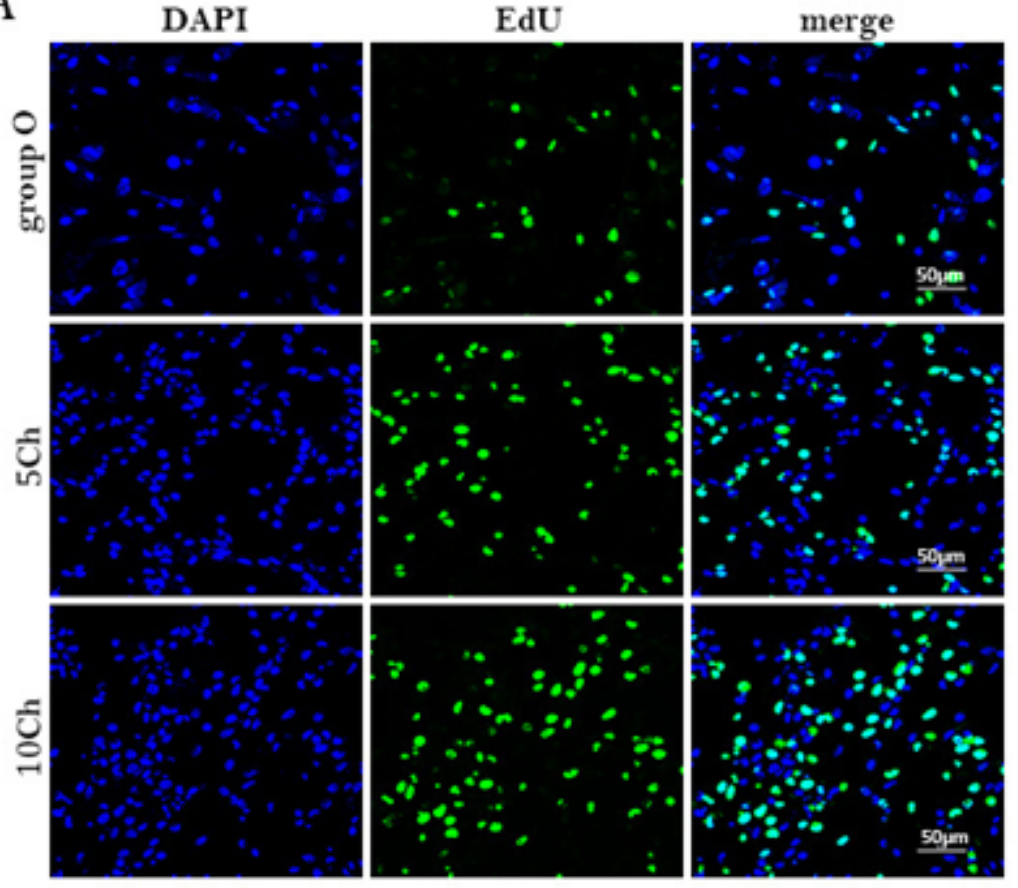

D

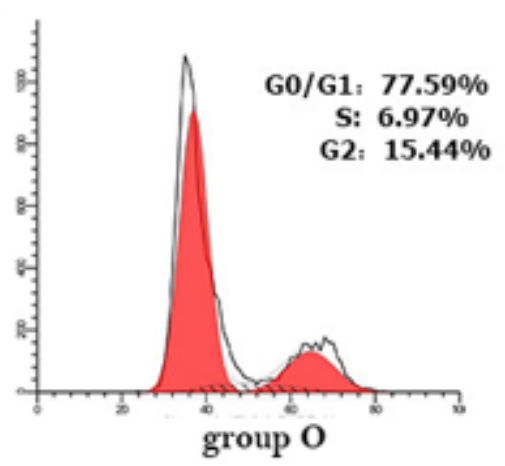

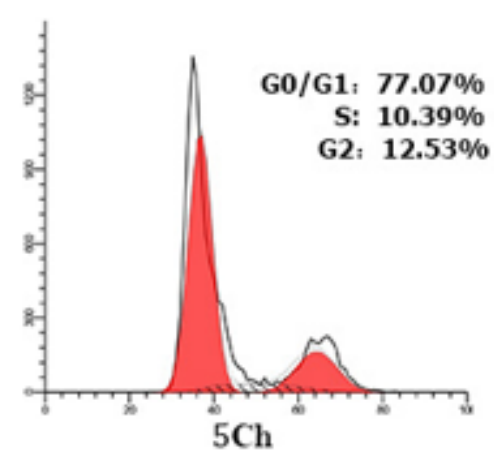

B

C
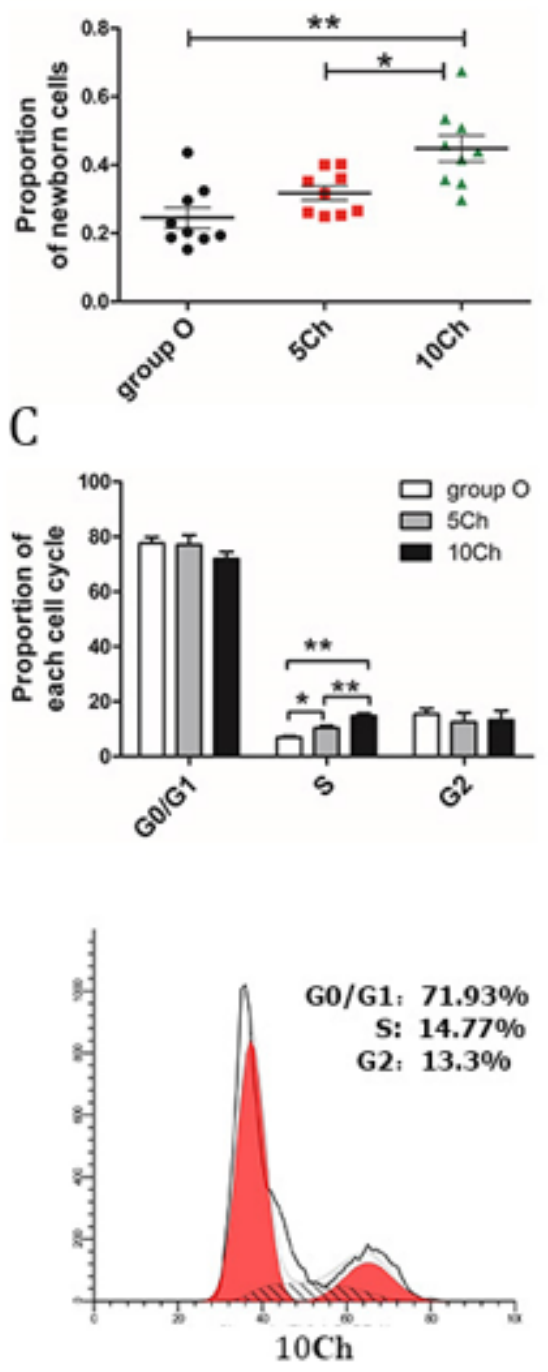

Figure 6

Chi3l1 can promote the proliferation of OPCs. 
A
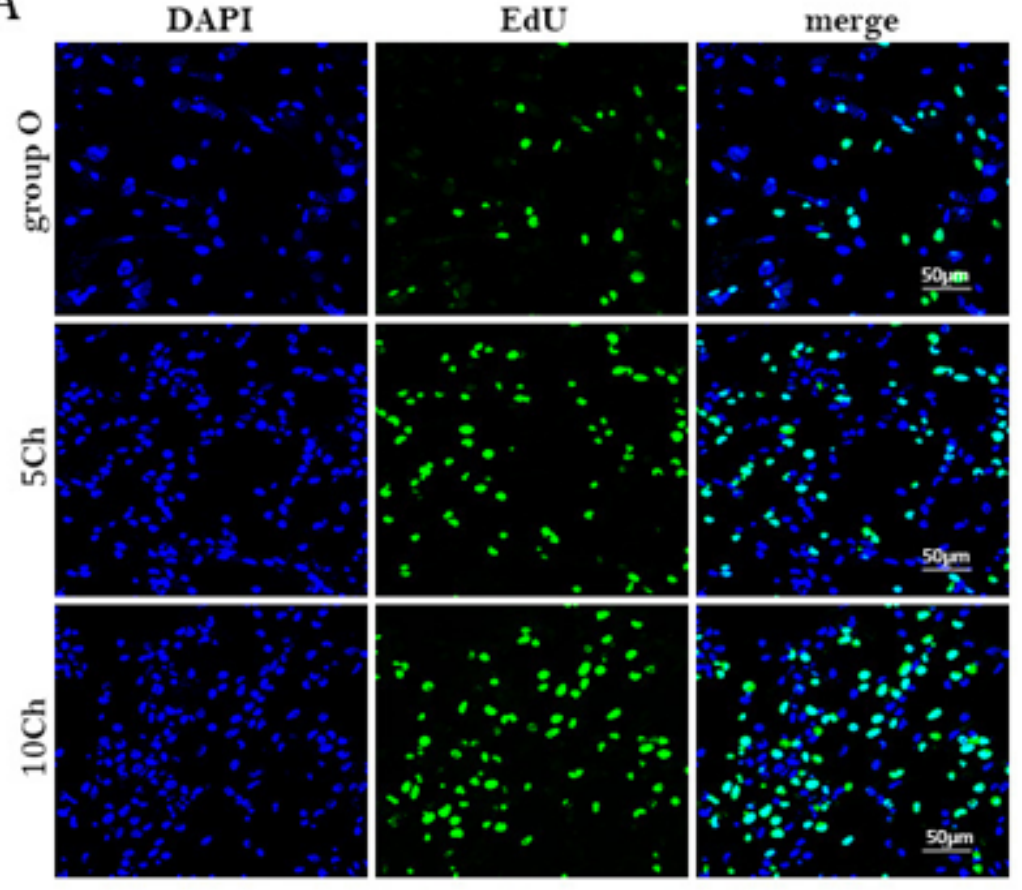

D

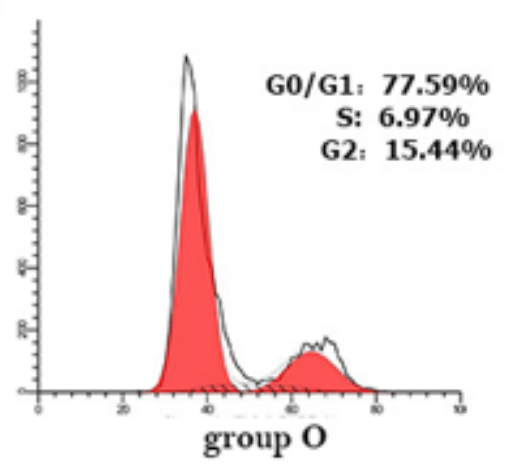

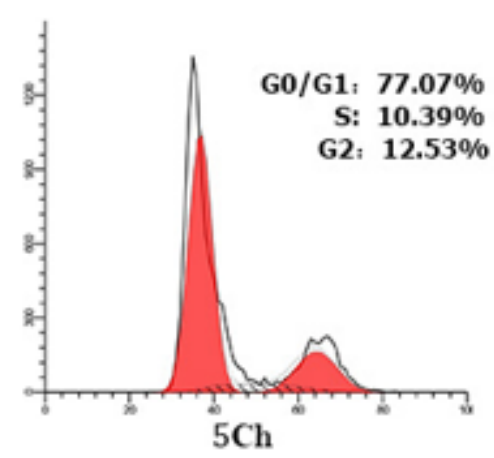

B

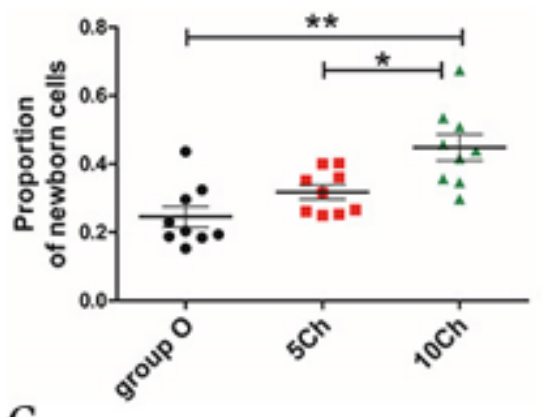

C
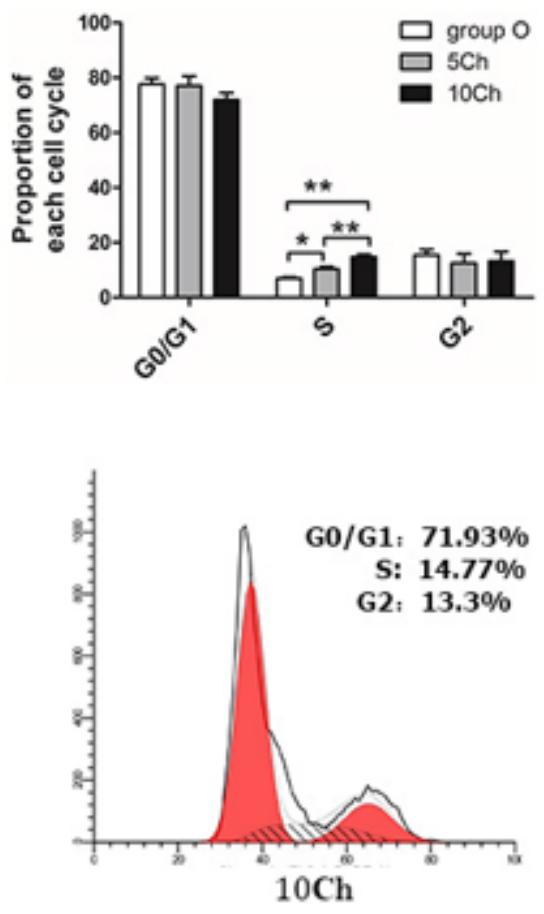

Figure 6

Chi3l1 can promote the proliferation of OPCs. 
A
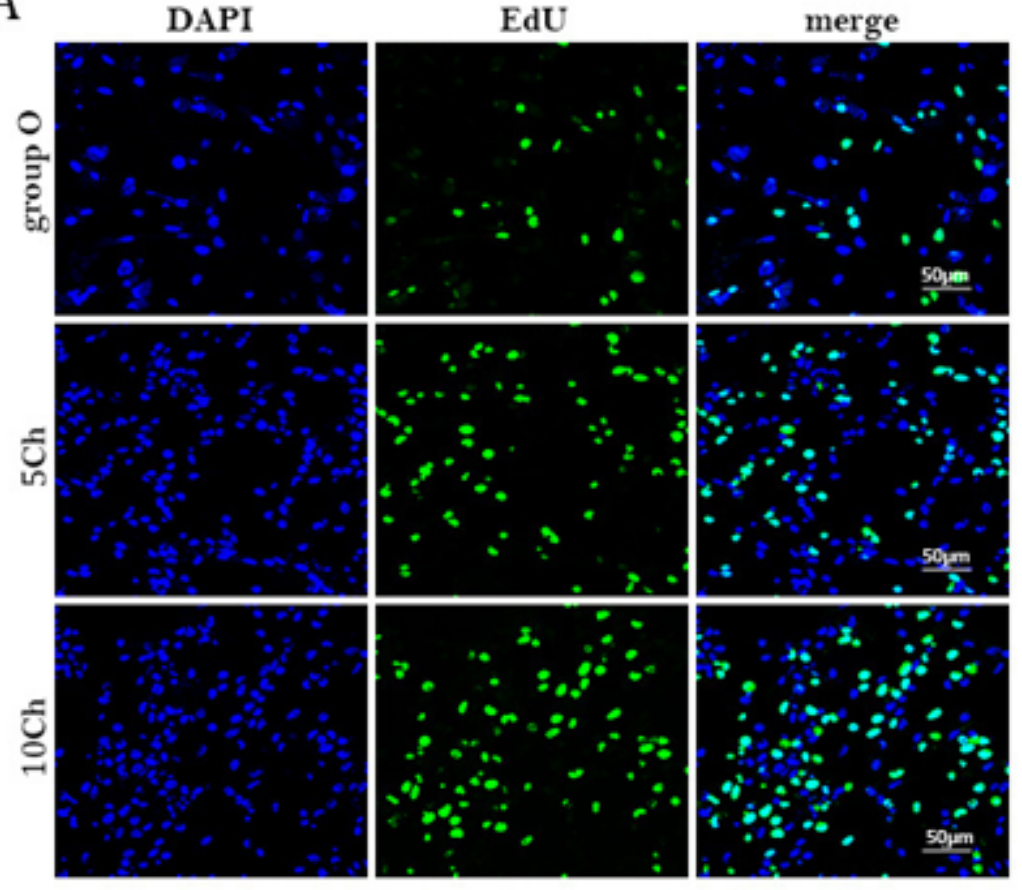

D

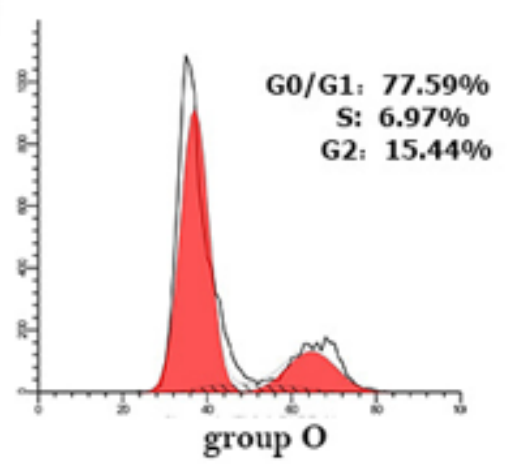

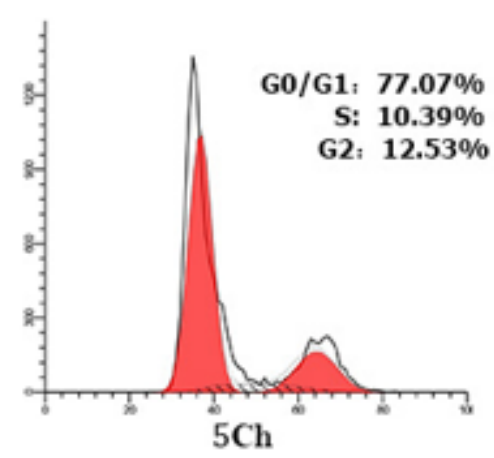

B

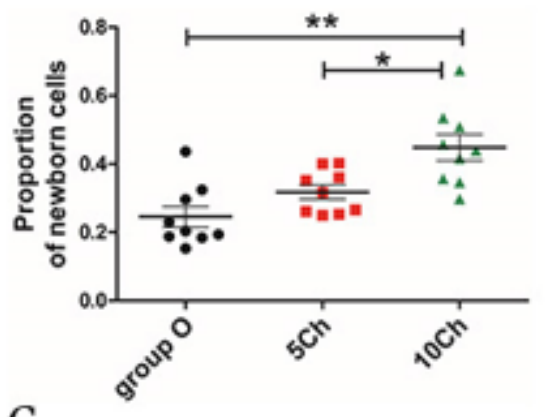

C
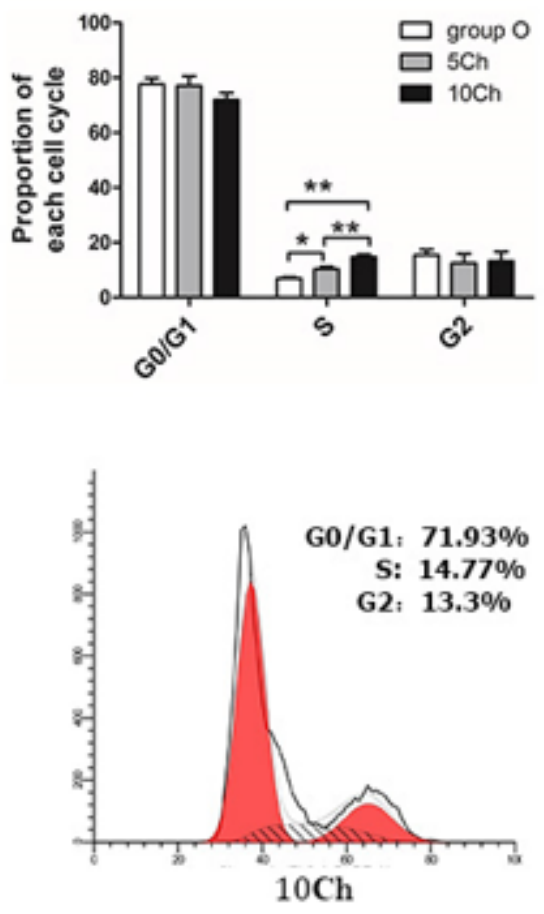

Figure 6

Chi3l1 can promote the proliferation of OPCs. 
A
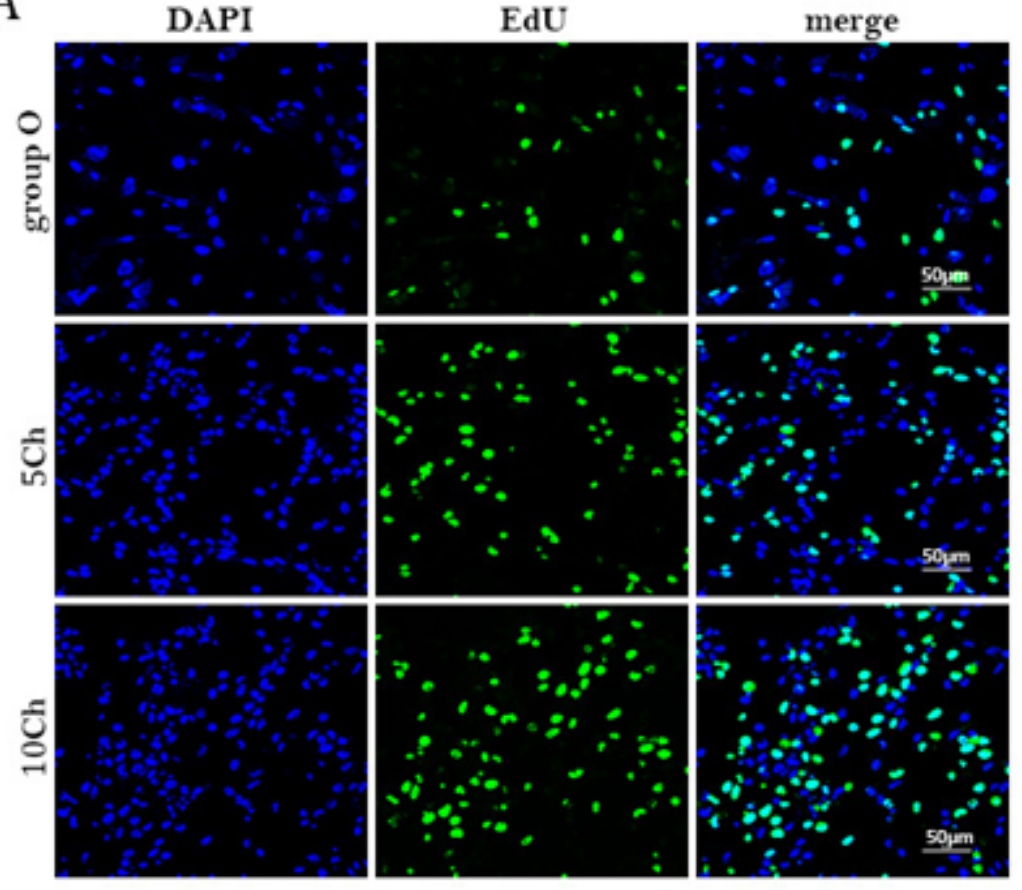

D

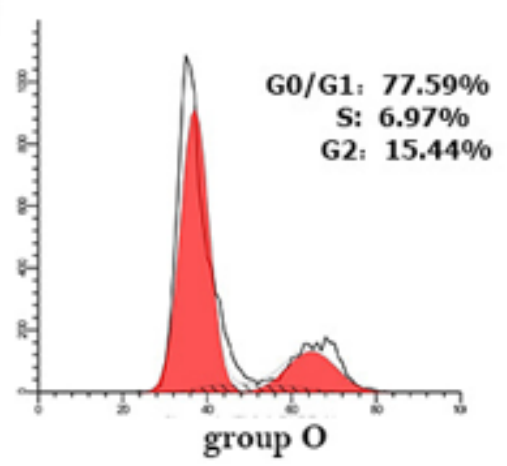

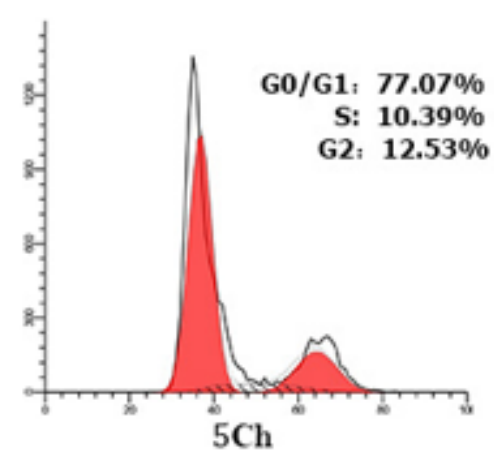

B

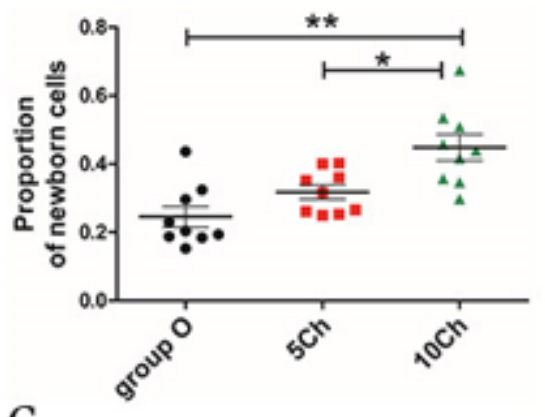

C
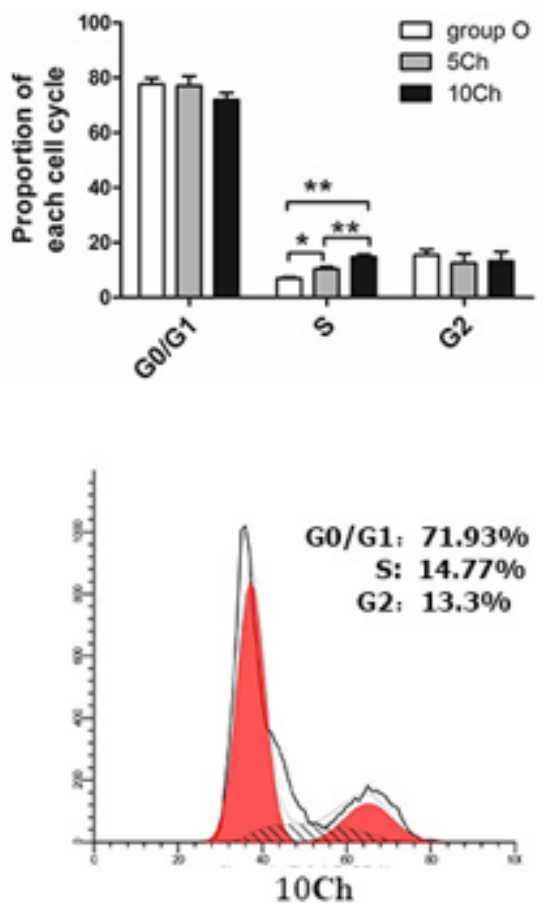

Figure 6

Chi3l1 can promote the proliferation of OPCs. 
A
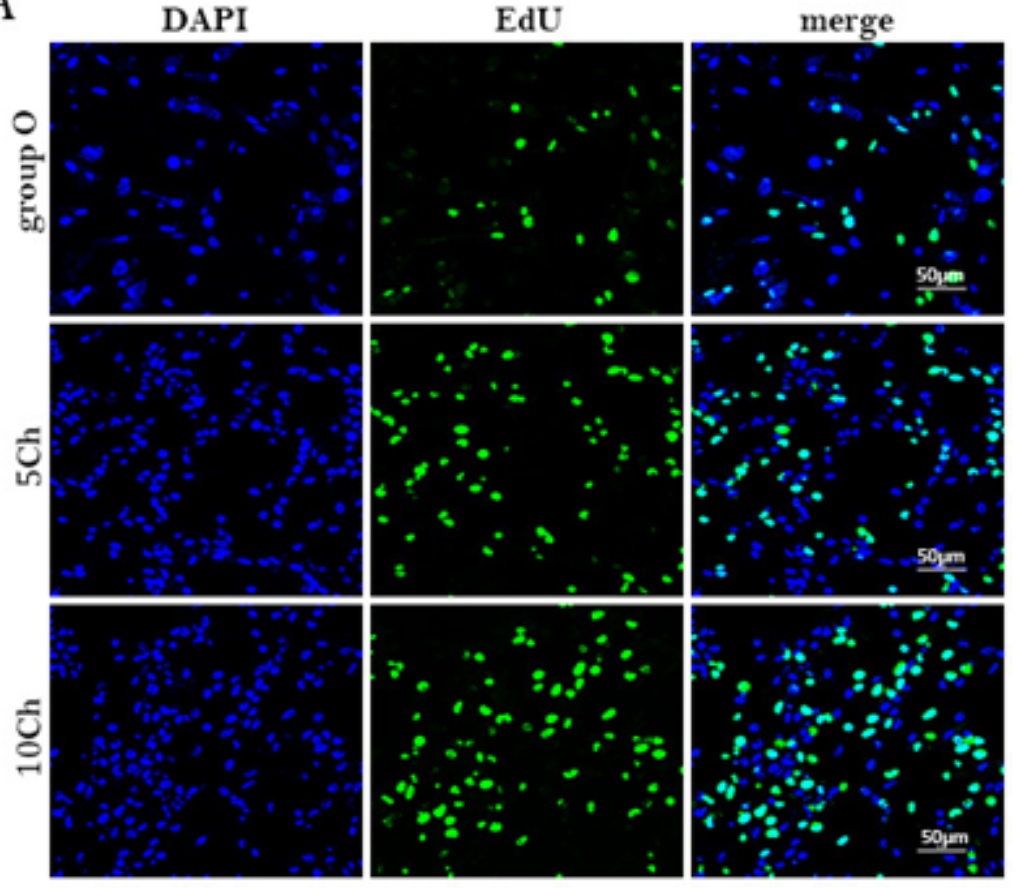

D

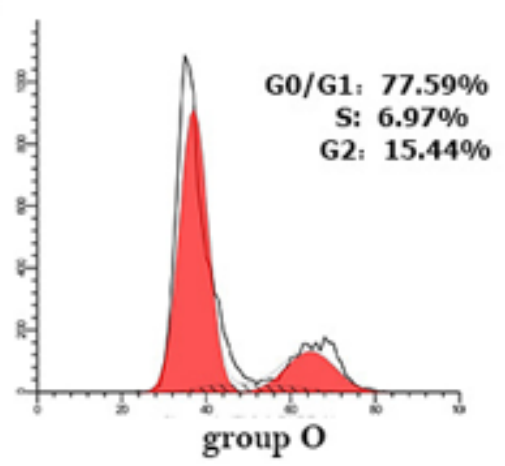

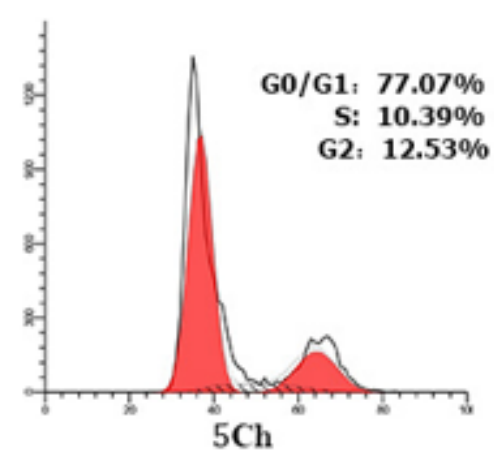

B

C
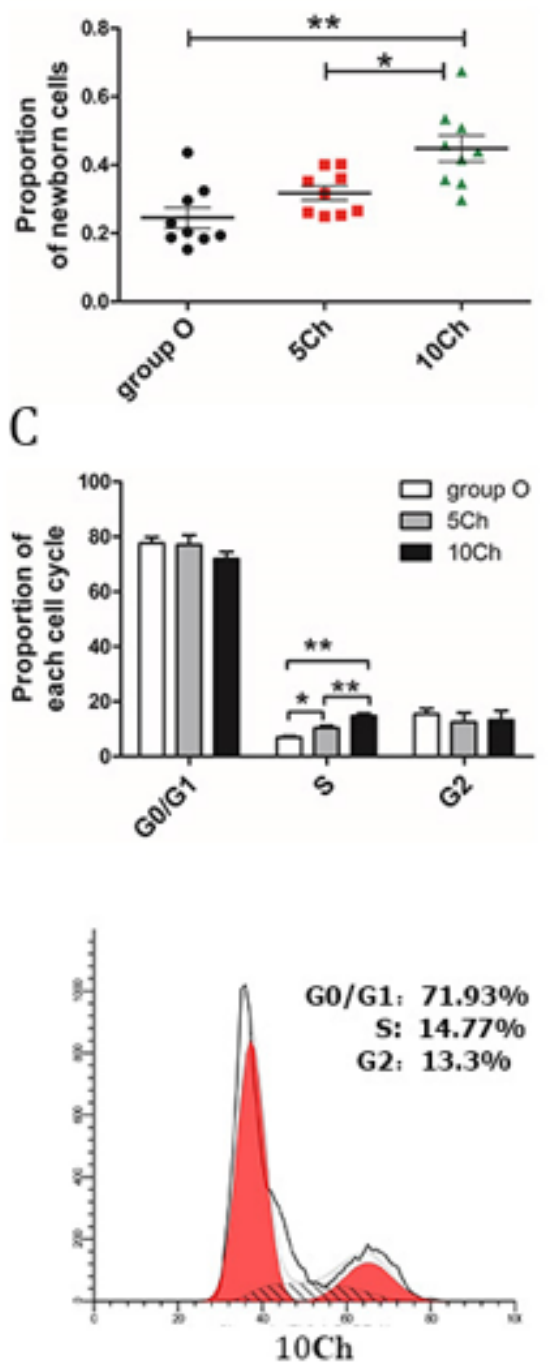

Figure 6

Chi3l1 can promote the proliferation of OPCs. 
A

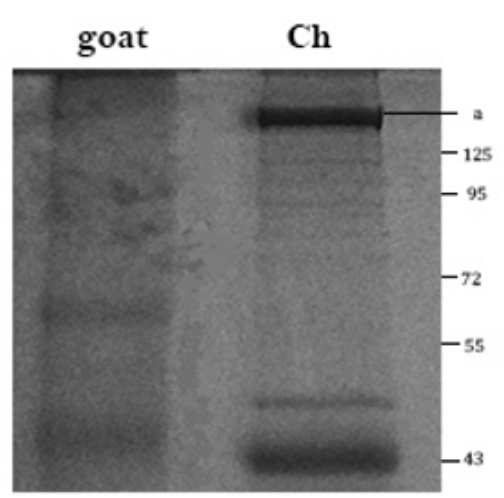

C

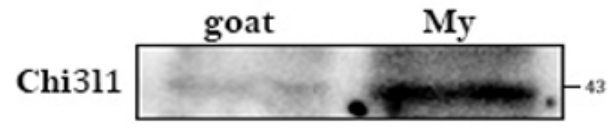

B

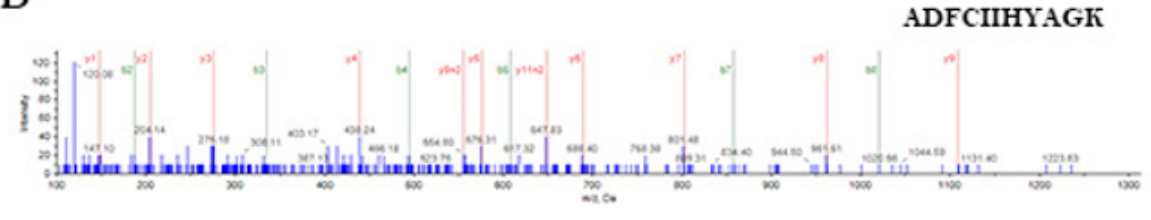

AGKLDPHLVLDQLR

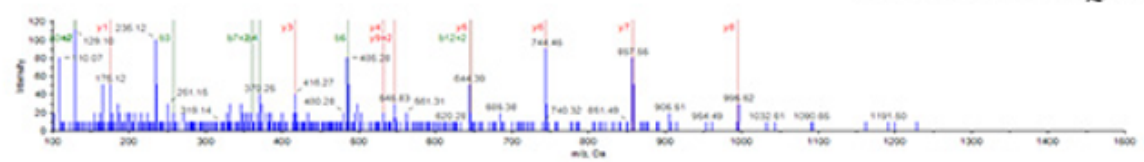

ASREEILAQAKENEK

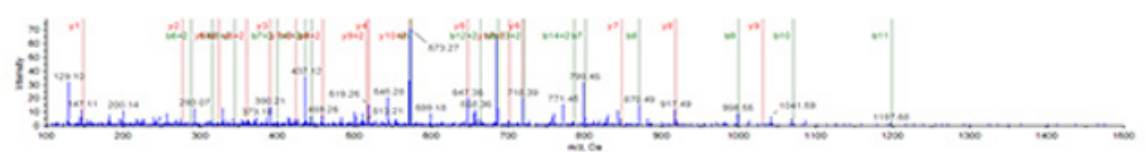

\section{Figure 7}

Mass spectrometry and Co-IP show Chi3l1 binding Myh9.

A

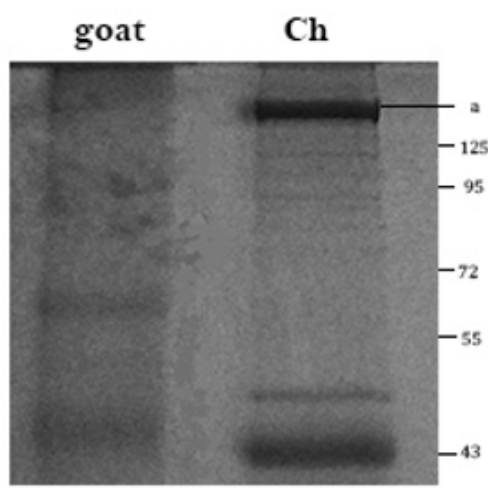

C

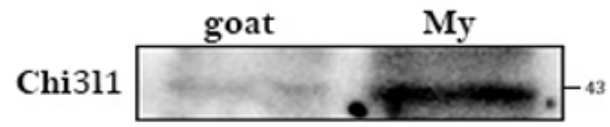

B ADFCIIHYAGK

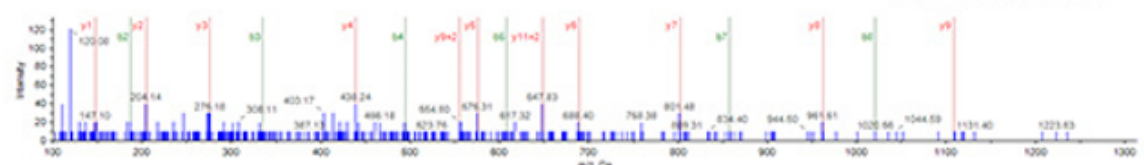

AGKLDPHLVLDQLR

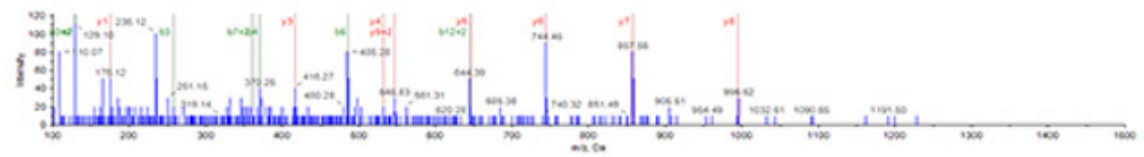

ASREEILAQAKENEK

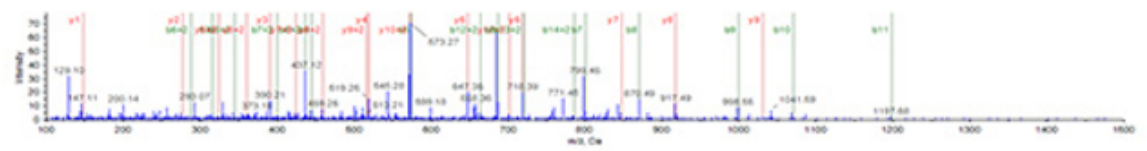

Figure 7

Mass spectrometry and Co-IP show Chi3l1 binding Myh9. 
A

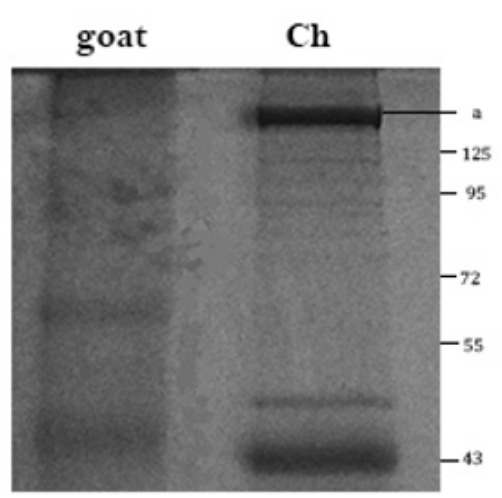

C

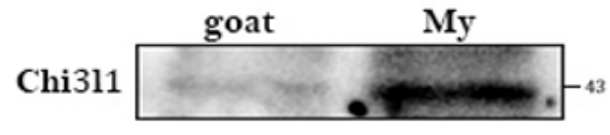

B

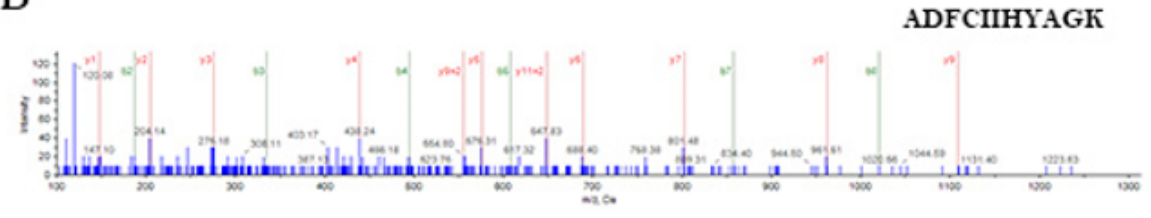

AGKLDPHLVLDQLR

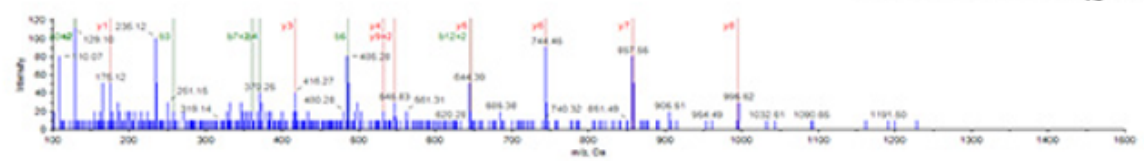

ASREEILAQAKENEK

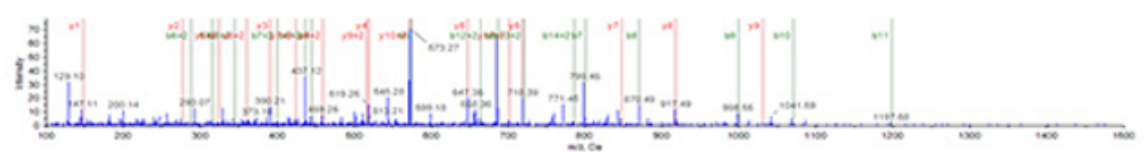

\section{Figure 7}

Mass spectrometry and Co-IP show Chi3l1 binding Myh9.

A

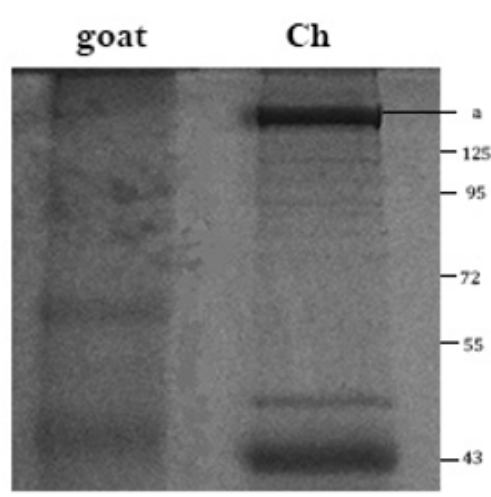

C

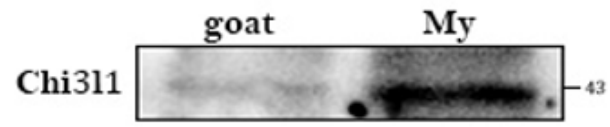

B ADFCIIHYAGK

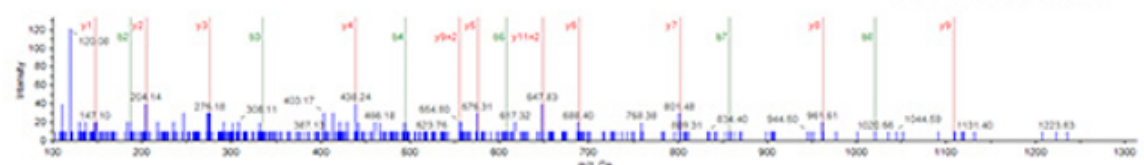

AGKLDPHLVLDQLR

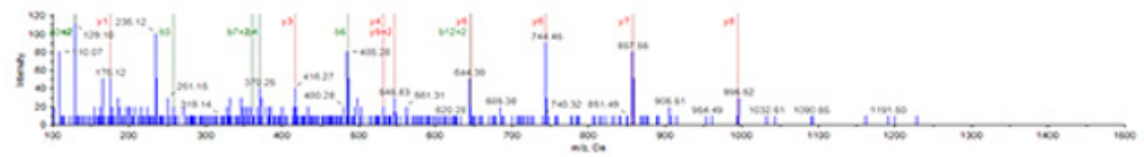

ASREEILAQAKENEK

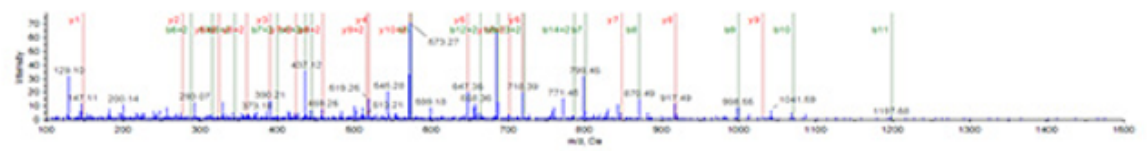

Figure 7

Mass spectrometry and Co-IP show Chi3l1 binding Myh9. 
A

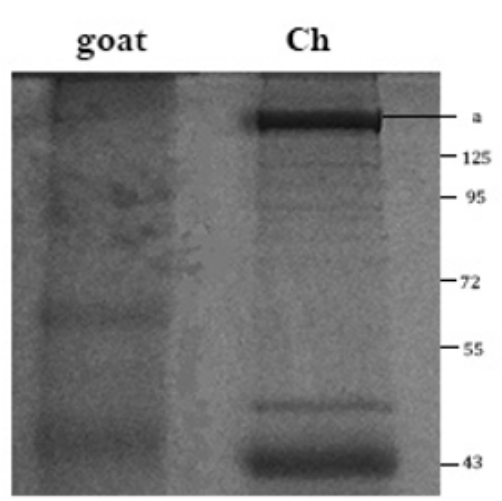

C

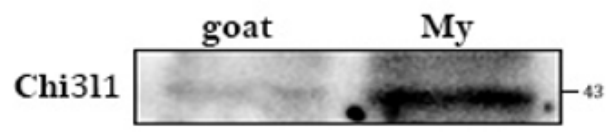

B

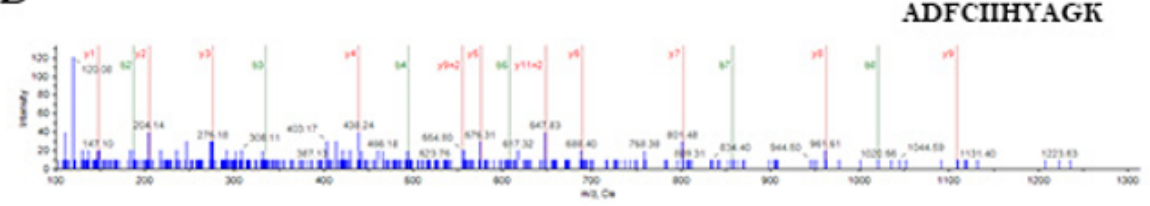

AGKLDPHLVLDQLR

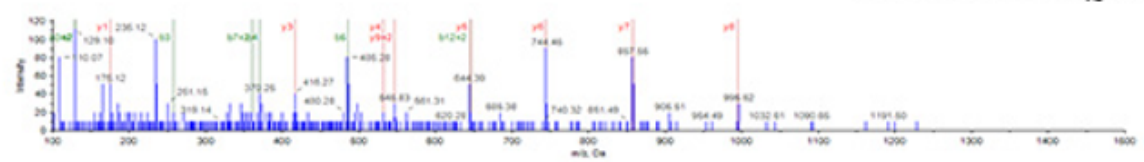

ASREEILAQAKENEK

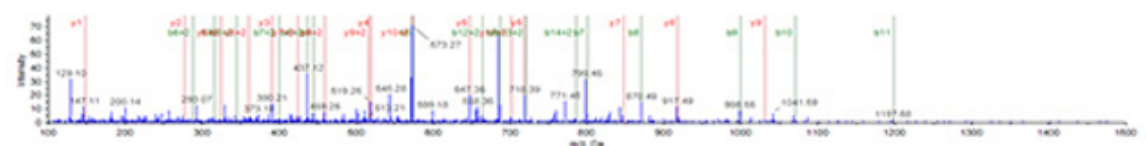

\section{Figure 7}

Mass spectrometry and Co-IP show Chi3l1 binding Myh9.

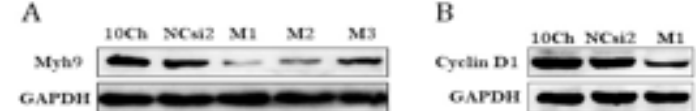

C

D
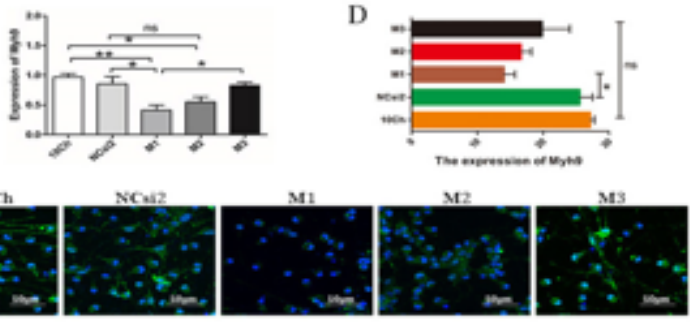

F

DAPI
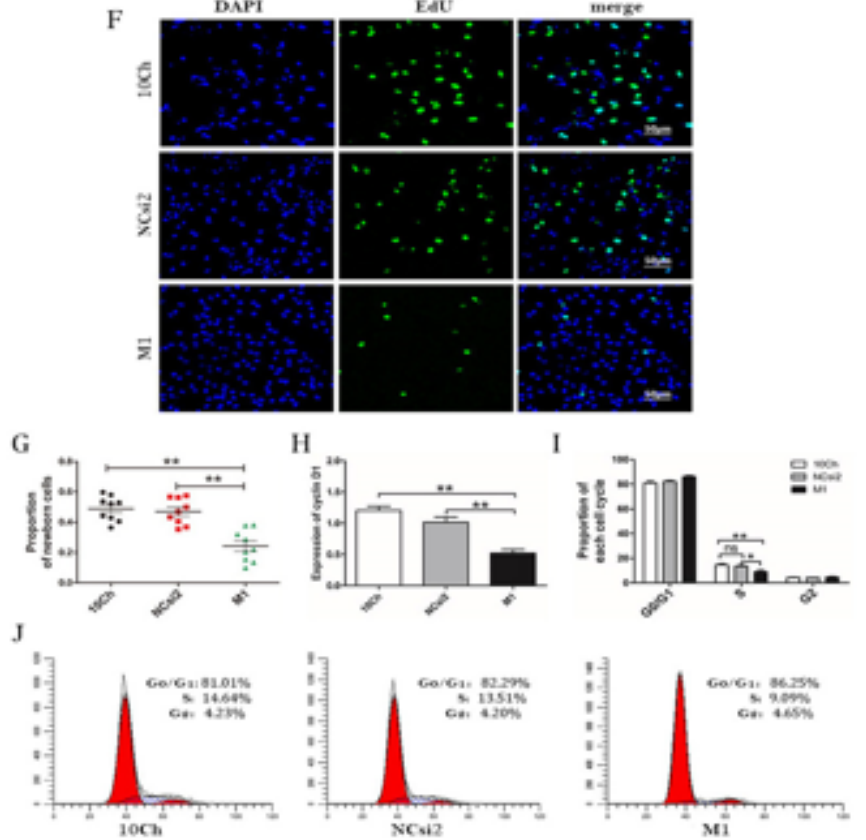
Figure 8

Chi3l1 promotes proliferation of OPCs through Myh9.
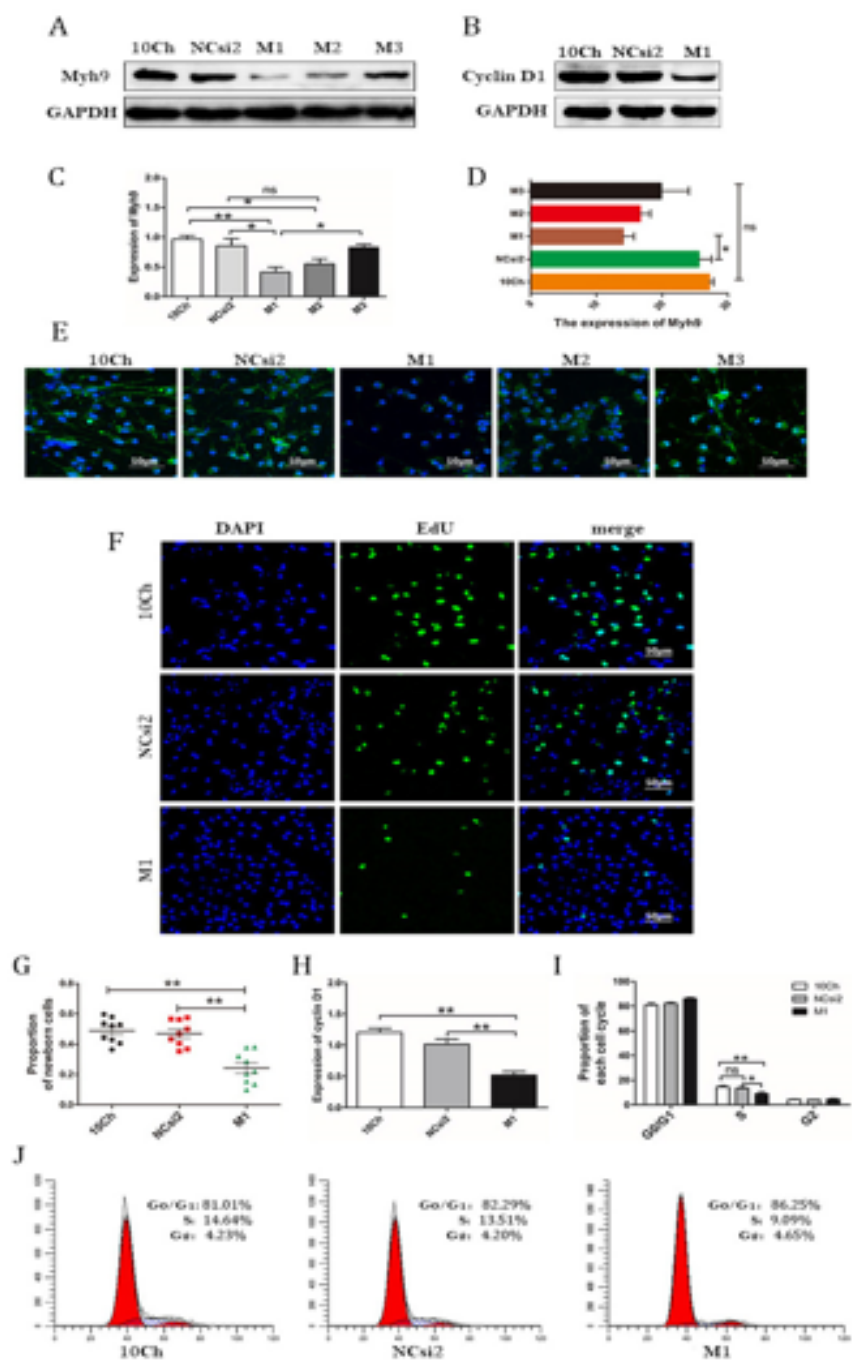

Figure 8

Chi3l1 promotes proliferation of OPCs through Myh9. 

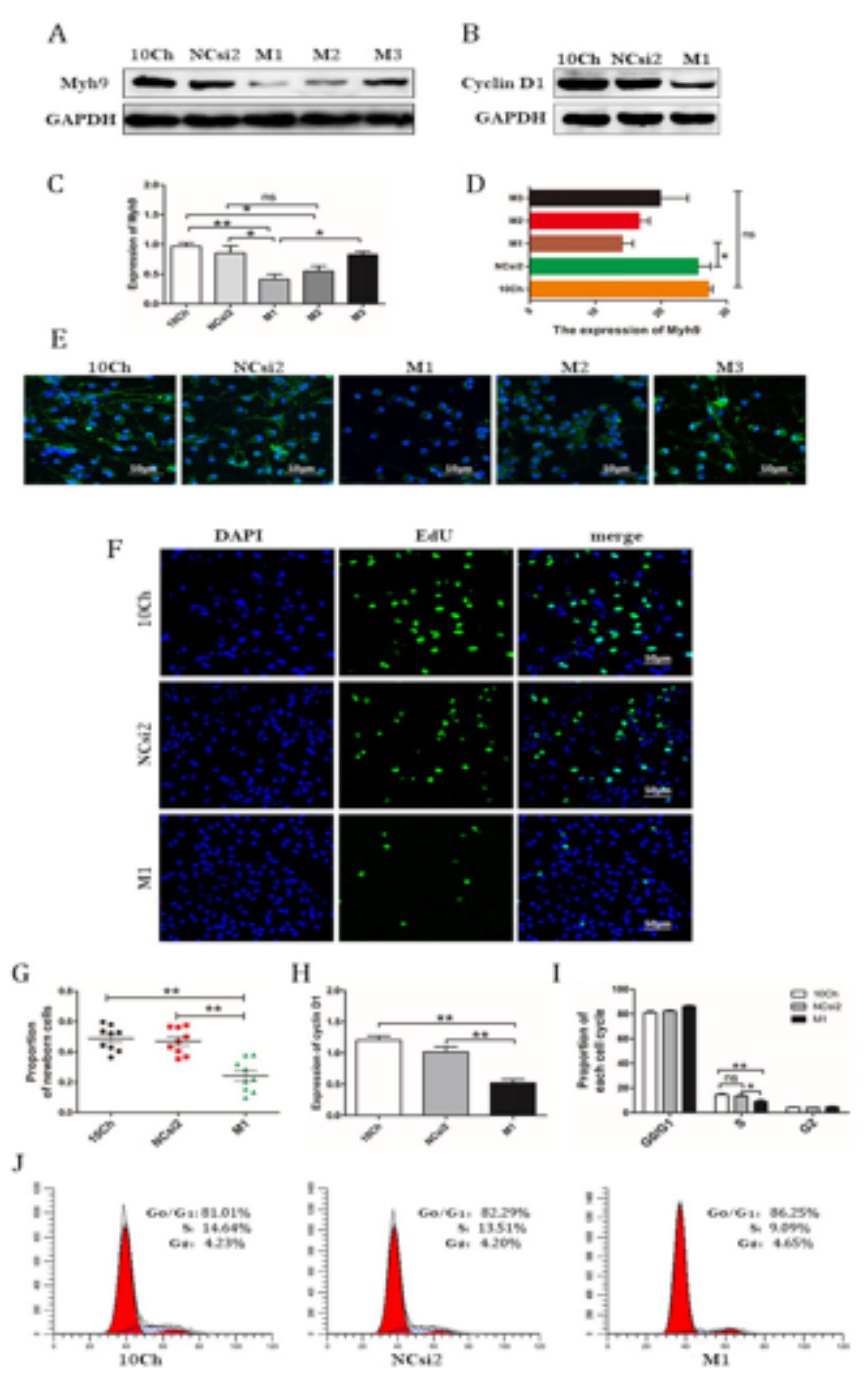

Figure 8

Chi3l1 promotes proliferation of OPCs through Myh9. 

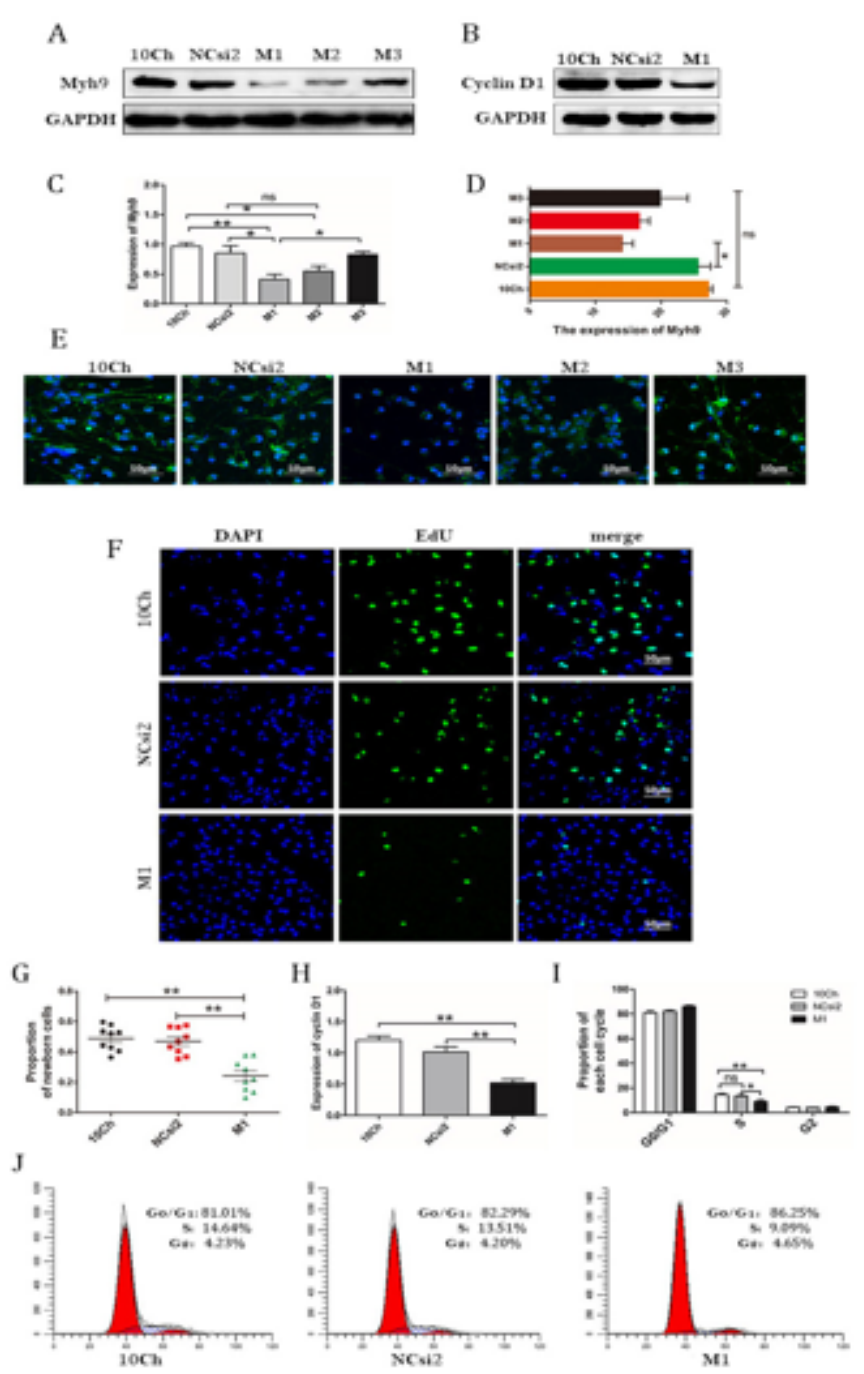

Figure 8

Chi3l1 promotes proliferation of OPCs through Myh9. 

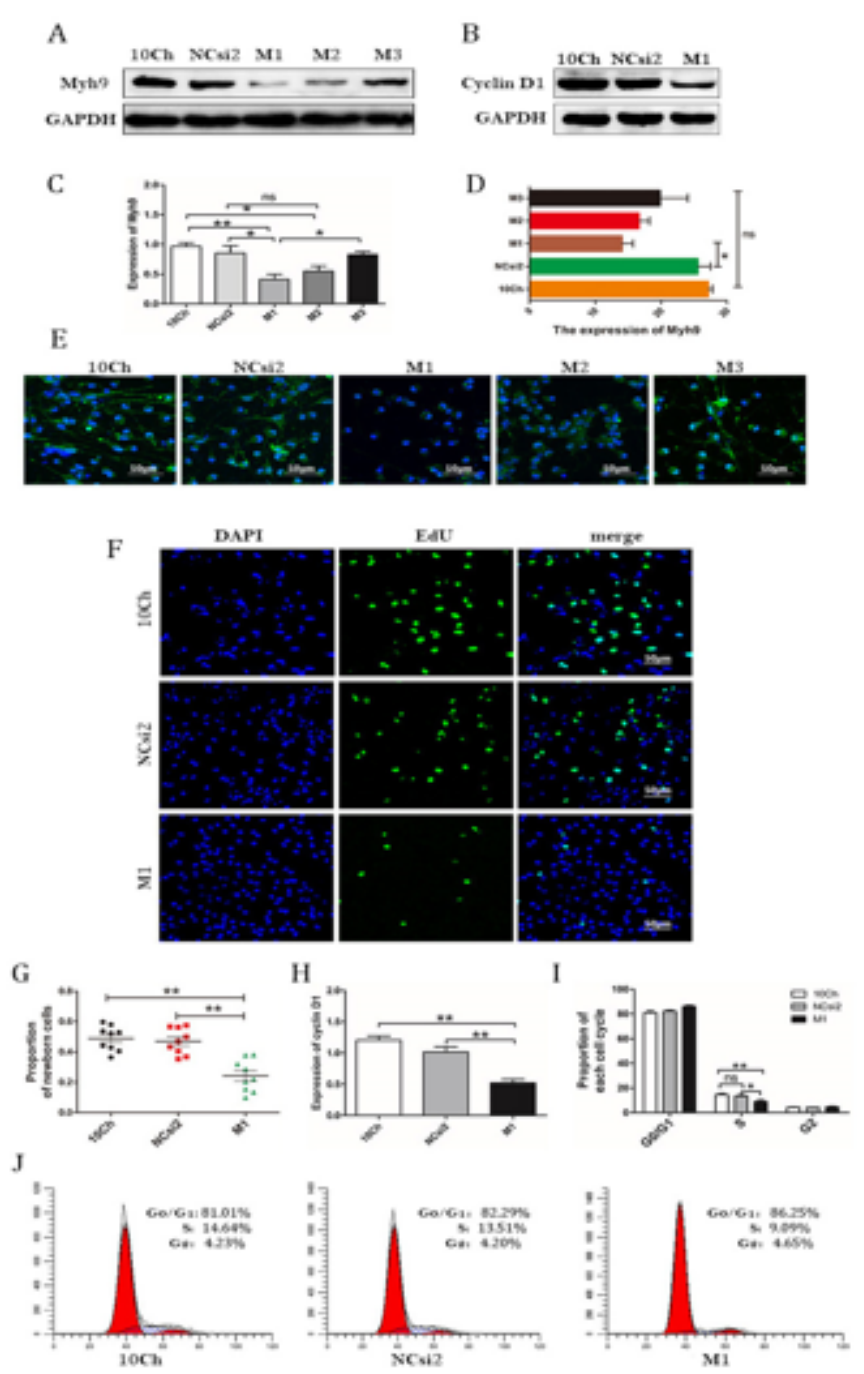

Figure 8

Chi3l1 promotes proliferation of OPCs through Myh9. 


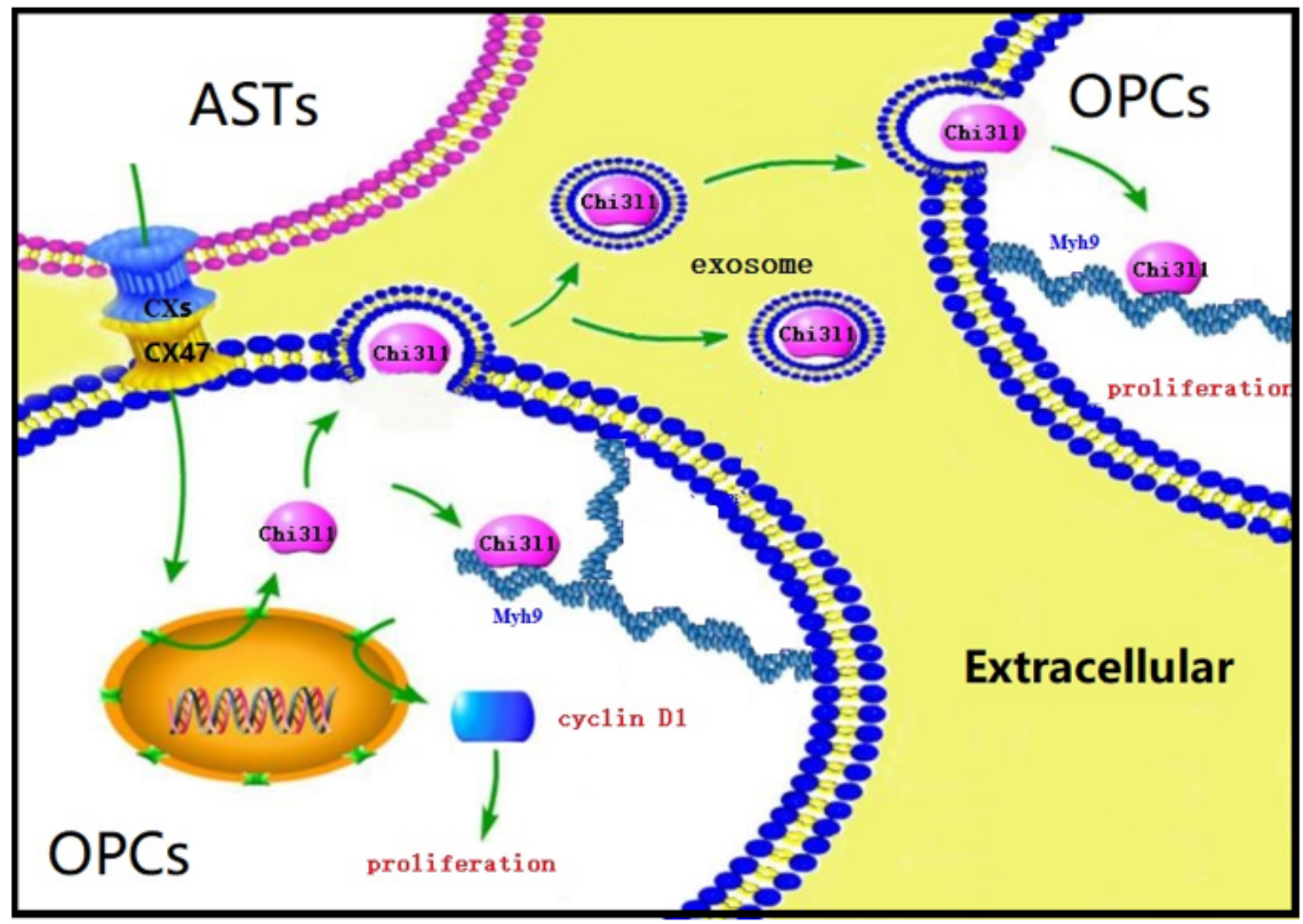

Figure 9

The signaling pathway of Chi3/1 induces OPCs proliferation. 


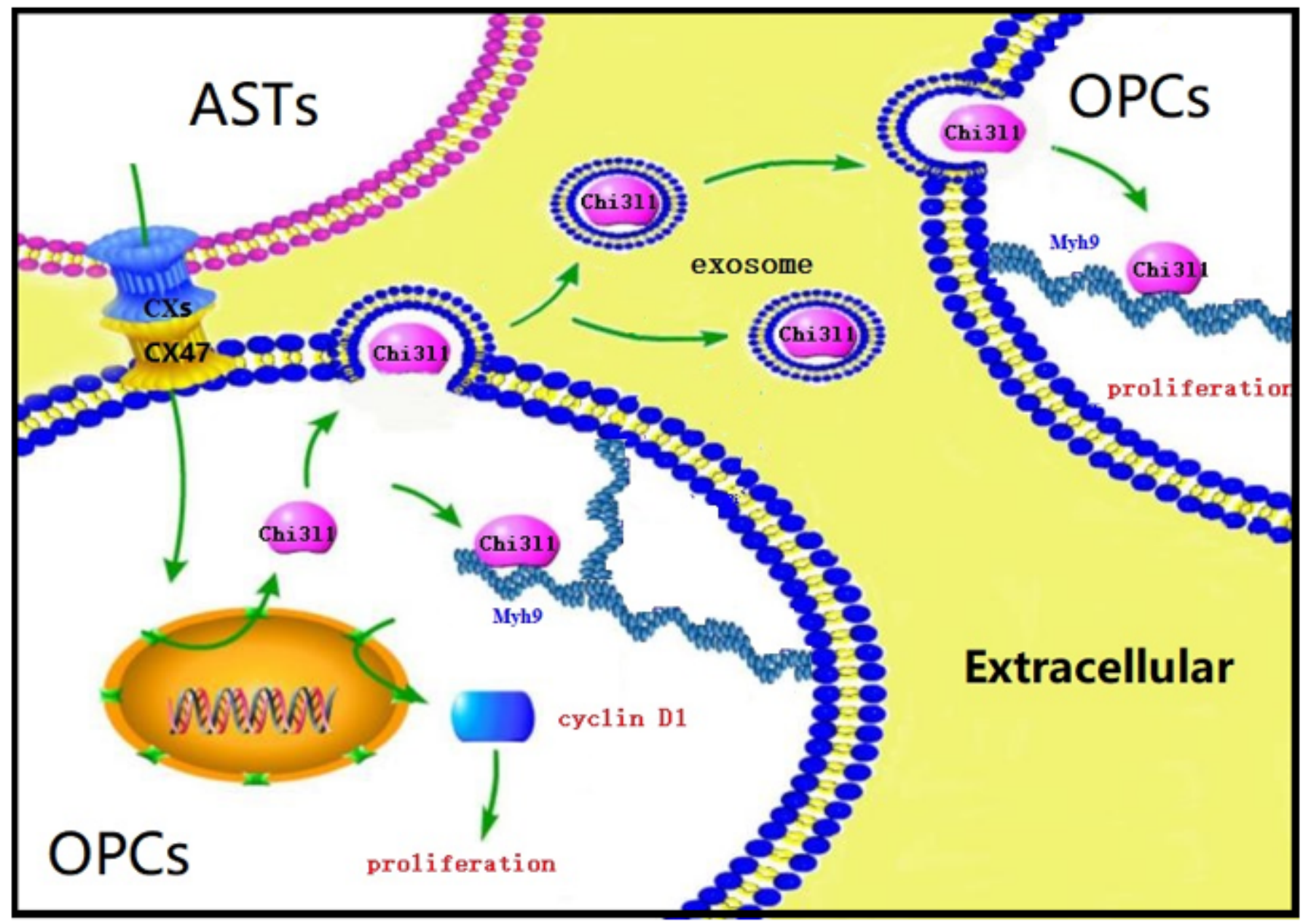

Figure 9

The signaling pathway of Chi3/1 induces OPCs proliferation. 


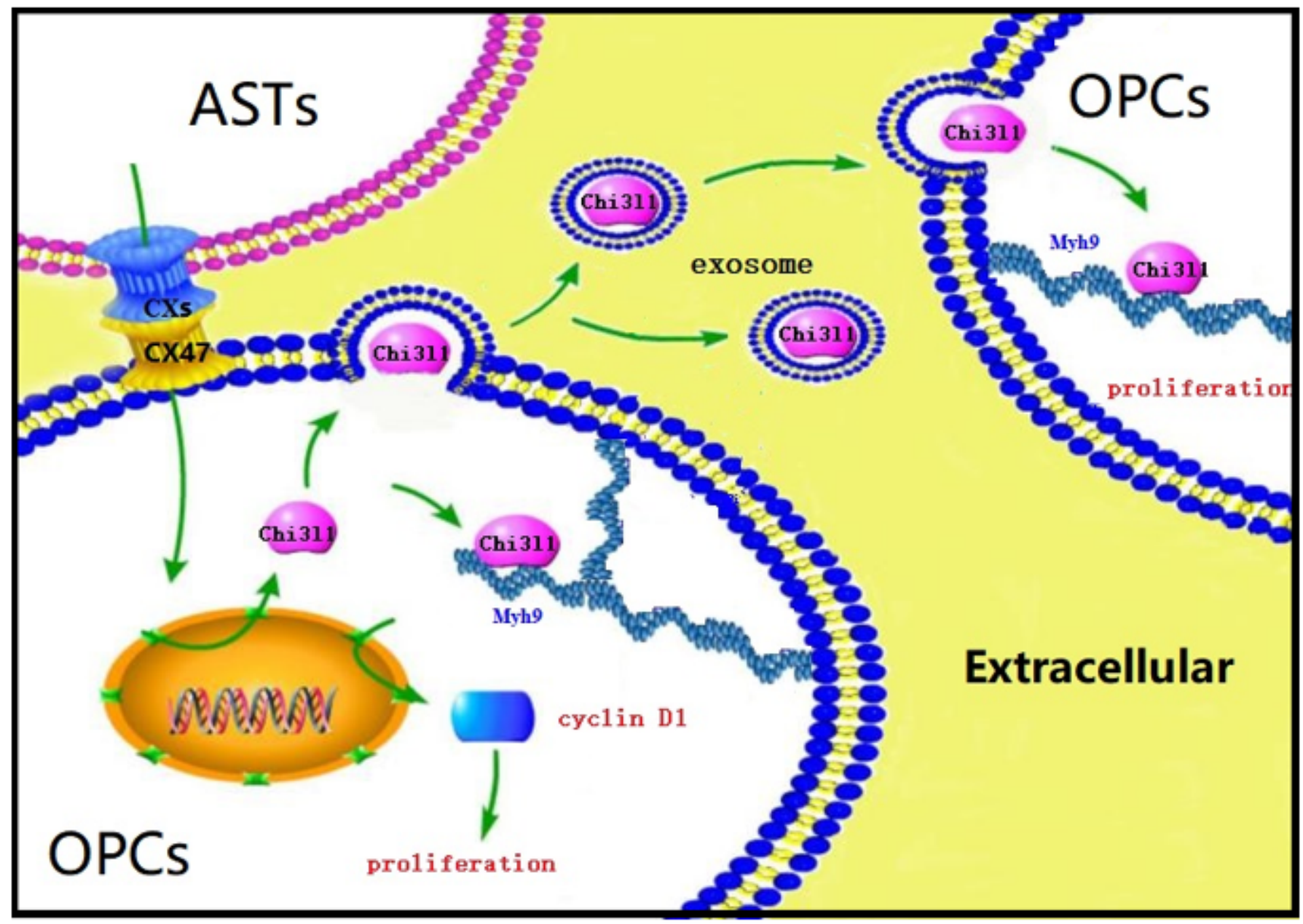

Figure 9

The signaling pathway of Chi3/1 induces OPCs proliferation. 


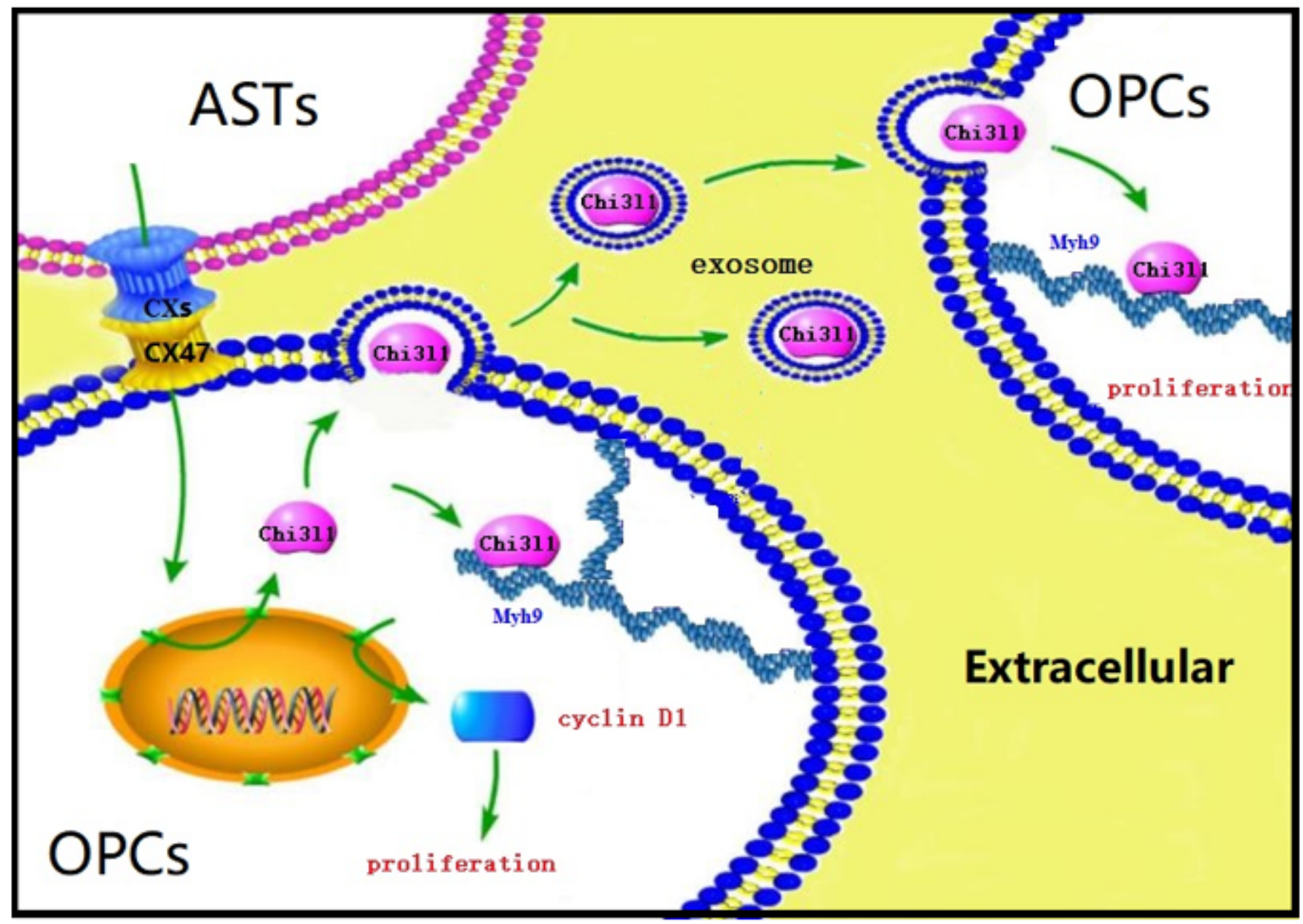

Figure 9

The signaling pathway of Chi3/1 induces OPCs proliferation. 


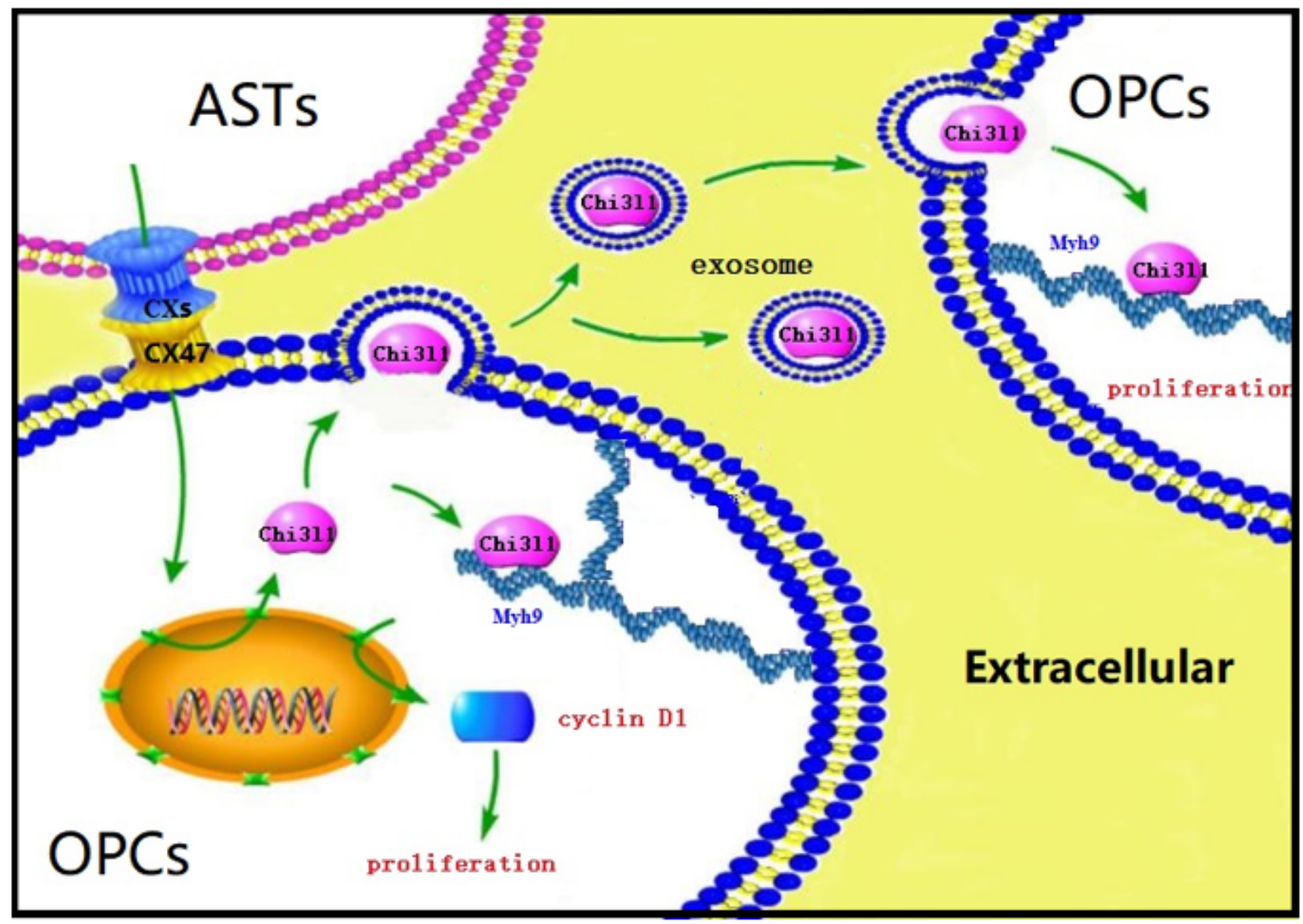

Figure 9

The signaling pathway of Chi3/1 induces OPCs proliferation. 